\title{
Acknowledgement to Reviewers of Molecules in 2018
}

\author{
Molecules Editorial Office \\ MDPI, St. Alban-Anlage 66, 4052 Basel, Switzerland \\ Published: 8 January 2019
}

\begin{abstract}
Rigorous peer-review is the corner-stone of high-quality academic publishing. The editorial team greatly appreciates the reviewers who contributed their knowledge and expertise to the journal's editorial process over the past 12 months. In 2018, a total of 3412 papers were published in the journal, with a median time to first decision of 12 days and a median time to publication of 31 days. The editors would like to express their sincere gratitude to the following reviewers for their cooperation and dedication in 2018:
\end{abstract}

Aav, Riina

Abadal, Sergi

Abarbri, Mohamed

Abashev, George G.

Abbamondi, Gennaro Roberto

Abbate, Sergio

Abbott, David H.

Abdalla, Maher Y.

Abdeeva, A. I.

Abdel-Daim, Mohamed

Abdelwahed, Sameh

Abdulkader, Fernando

Abenavoli, Ludovico

Abeywardana, Tharindumala

Abraham, Wolf-Rainer

Abuillan, Wasim

Abul-Haija, Yousef

Abu-Reidah, Ibrahim M.

Abu-Romman, Saeid

Abuzaid, Ahmed

Accardo, Antonella

Acero, Nuria

Achaerandio Puente, Maria Isabel

Acharya, Priyamvada

Achelle, Sylvain

Acherar, Samir

Achilias, Dimitris S.

Achour, Brahim

Acosta Contreras, Florentina Niuris

Acquah, Gifty E.

Acuña-Castroviejo, Darío

Adachi, Naoya

Adam, Vojtěch

Adamcik, Jozef

Adamczyk, Bartosz

Adamczyk-Woźniak, Agnieszka
Adams, James David

Addepalli, Balasubrahmanyam

Adhikari, Khem Bahadur

Adhikari, Upendra

Adriana, Estrada-Bernal

Adrover, Alessandra

Afantitis, Antreas (Cyprus)

Afantitis, Antreas (Greece)

Afarinkia, Kamyar

Afonin, Andrei V.

Afonin, Kirill

Afzal, Adeel

Agamennone, Mariangela

Agarwal, Sumit

Agarwal, Vinayak

Agatemor, Christian

Ageitos, Jose Manuel

Agliari, Elena

Agnieszka, Szopa

Agostino, Mark

Agrawal, Prashansa

Agrawal, Siddarth

Agriopoulou, Sofia

Aguilar, Carme

Aguilar, Enrique

Agustin, Dominique

Agyei, Dominic

Ahmad, Zeeshan

Ahmadi-Afzadi, Masoud

Ahmed, Alauddin

Ahmed, Marya

Ahmed, Sahar

Ahn, Heekwon

Ahn, Ji-Young

Ahvazi, Behzad

Aiba, Yuichiro 
Aich, Nirupam

Aiello, Francesca

Aigbirhio, Franklin I.

Aimanianda, Vishukumar

Aires, Alfredo

Aitken, R. Alan

Aivaras Kareiva, Habil

Akamatsu, Miki

Akanbi, Taiwo

Akens, Margarete

Akitsu, Takashiro

Akk, Gustav

Alabugin, Igor V.

Alaimo, Salvatore

Alam, Md Badrul

Alam, Mohammad Abrar

Alam, Mohammad Parvez

Alasbahi, Bandar

Alashi, Adeola M

Albanese, Domenico

Albergamo, Ambrogina

Albergante, Luca

Albericio, Fernando

Alberto, Roger

Albiniak, Philip

Albo, Jonathan

Albuquerque, Magaly

Aldabbagh, Fawaz

Alder, Jane

Alegre, Cinthia

Alexander, Bruce D

Alexis, Frank

Alexopoulos, Athanassios

Alfano, Alberto

Alfano, Daniela

Al-Fatimi, Mohamed

Alferink, Judith

Alhazmi, Hassan A.

Al-Horani, Rami

Alizadeh, Hossein

Alkorta, Ibon

Allain, Fabrice

Allais, Florent

Allinquant, Bernadette

Allsopp, Luke P

Almajano, María Pilar

Almeida, Henrique

Almendros, Gonzalo

Almoazen, Hassan

Alonso, Diego A.

Alonso, Leocadio

Alonso, Rosa Maria
Al-Rimawi, Fuad

Altemimi, Ammar

Altieri, Fabio

Altmann, Friedrich

Alvarez, Carlos

Álvarez, Joaquín

Alvarez, Laura

Álvarez-Fernández, M. Antonia

Alvarez-Idaboy, Juan Raùl

Alvarez-Suarez, José

Alves, Carlos Roberto

Alves, Eliana

Alves, Harley Da Silva

Alves, Maria José

Alves, Vítor $\mathrm{H}$.

Amano, Masafumi

Amarowicz, Ryszard

Amato, Jussara

Amato, Rosario

Amelin, Vasiliy G.

Amengual, Jaume

Amgoune, Abderrahmane

Ami, Diletta

Amigo-Benavent, Miryam

Amii, Hideki

Amin, M. Junaid

Amirkhani, Masoume

Ammendola, Rosario

Amodeo, Pietro

Amorati, Riccardo

Amorós, Asunción

Amrutkar, Manoj

Amsharov, Konstantin

An, Yu

Anadón, Arturo

Anand, Vijay

Anas, Andrea Roxanne

Ancuceanu, Robert

Ancuceanu, Robert Viorel

Andersson, Håkan S

Andjelkovic, Mirjana

Ando, Kaori

Andolfi, Anna

Andrade, Fernando

Andrade, Isabel

Andrade, Paula B

Andrea, Penoni

Andreana, Peter

Andrés-Sánchez, Santiago

Andrésson, Ólafur S.

Andrianasolo, Eric

Andrić, Filip 
Andruszkiewicz, Ryszard

Anfossi, Laura

Angelico, Ruggero

Angelova, Violina T.

Angilella, Giuseppe G. N.

Angiolella, Letizia

Anna, Szakal

Annor, George

Anraku, Makoto

Antal, Diana

Antanasijević, Jelena

Anticó, Enriqueta

Antoni, Gunnar

Anuta, Valentina

Anzai, Jun-ichi

Anzenbacher, Pavel

Aoki, Dan

Apartsin, Evgeny

Apetrei, Constantin

Aprea, Eugenio

Aprodu, Iuliana

Aquaro, Stefano

Aragó, Juan

Arai, Toshiro

Araki, Kazuo

Arambula, Jonathan

Arancibia, Rodrigo

Arango, Rachel A.

Araniciu, Cătălin

Arano, Yasushi

Arcadi, Antonio

Archibald, Steve

Arguelles, Sandro

Arion, Vladimir

Arita, Masanori

Armentrout, Peter B.

Arnao, Marino B

Arnason, John

Arrachart, Guilhem

Arroo, Randolph

Arroyo, Netzahualcóyotl

Arteaga, Jesús F.

Artetxe, Beñat

Artola, Marta

Artyszak, Arkadiusz

Arvapally, Ravi Kumar

Asai, Daisuke

Asakawa, Yoshinori

Asamizu, Shumpei

Ascenzi, Paolo

Asfin, Ruslan E.

Ashley, Jon
Askes, Sven

Asmontas, Steponas

Aspatwar, Ashok

Assari, Shervin

Assfeld, Xavier

Åstrand, Alexander

Atanasova-Penichon, Vessela

Atef, Eman

Atobe, Masakazu

Attanasio, Chiara

Aubert, Emmanuel

Audette, Gerald F

Auvynet, Constance

Avato, Pinarosa

Avci, Fikri

Avdeeva, Varvara V.

Averin, Alexei D.

Aviles, Francesc Xavier

Avin, Vijay

Awale, Suresh

Ayala-Zavala, J. F.

Azab, Abdullatif

Aziz, Athar

Azman, Samet

B. Luwor, Rodney

Baba, Hiromi

Babaev, Eugene

Babii, Oleg

Baby, André

Bacelar, Eunice

Bach, Duc-Hiep

Back, Kyoungwhan

Bacolla, Albino

Bácskay, Ildikó

Bacso, Zsolt

Bączek, Katarzyna

Bączek, Tomasz

Badawi, Mohamed

Baddeley, David

Bado, Igor

Bae, Jiyoung

Bae, Ki Hyun

Baek, Hyung Hee

Baek, Kwang-Hyun

Baek, Nam-In

Baeza, Alejandro

Bagdziunas, Gintautas

Bahn, Andrew

Bai, Fang

Bai, Mingfeng

Bai, Renren

Bai, Shiqiang 
Baier, Andrea

Bailly, Fabrice

Bajaj, Sumit

Bajpai, Richa

Bak, Andrzej

Bak, Istvan

Bakare, Oladapo

Bakillah, Ahmed

Baksh, Shairaz

Bakunina, Irina

Balan, Venkatesh

Balasubramaniam, Meenakshisundaram

Balasubramaniam, Sivaraman

Balbach, Jochen

Balcerek, Maria

Baldisserotto, Anna

Bálint, Erika

Baliraine, Frederick

Ballard, Nicholas

Ballmer-Hofer, Kurt

Bally, Julia

Balogh, György Tibor

Baltakys, Kęstutis

Balzer, Frank

Banach, Marcin

Banchero, Mauro

Bandar, Jeffrey S.

Bandyopadhyay, Debasish

Bandyopadhyay, Sulalit

Banerjee, Aditi

Banerjee, Kalpita

Banerjee, Sudip

Baniahmad, Aria

Bankina, Biruta

Bankova, Vassya

Banning, Antje

Banoub, Joseph

Banskota, Arjun H.

Bansode, Atul

Banti, Christina N.

Bantreil, Xavier

Bao, Ande

Bao, Yinyin

Bao, Yong-Ping

Barakat, Assem

Baraldi, Pier Giovanni

Baranski, Rafal

Barattucci, Anna

Barba, Anna A.

Barba, Francisco J.

Barbaric, Monika

Barbero, Gerardo Fernández
Barbieri, Antonio

Barbieri, Pierluigi

Barbillon, Grégory

Barchi, Joseph J.

Barczyński, Piotr

Bardají, Eduard

Bardelang, David

Barendt, Timothy

Barišić, Lidija

Barja-Fidalgo, Christina

Barker, Bridget $\mathrm{M}$.

Bar-Kohany, Tali

Barlocco, Daniela

Barman, Apurba

Barnych, Bogdan

Baron, Marco

Barra, Guilherme M.O.

Barradas, Thaís Nogueira

Barreira, João C.M.

Barrero, Carlos A

Barreto, Guillermo

Barreto, Maria Do Carmo

Barros, Antonio

Barros, Pedro M.

Barry, Cornelius

Barry-Ryan, Catherine

Bar-Shavit, Zvi

Bartelt, Alexander

Bartocci, Pietro

Barton, Larry L.

Bartoněk, Dalibor

Bartosz, Grzegorz

Bartoszewski, Rafal

Bartzas, Georgios

Baryshnikov, Glib

Basak, Ajit K.

Basak, Sujit

Basile, Teodora

Basini, Giuseppina

Basit, Abdul

Bassin, Paul

Bassoli, Angela

Basu, Swaraj

Batail, Patrick

Batista, Irineu

Battistelli, Cecilia

Batty, Kevin T.

Batule, Bhagwan Sahebrao

Bauer, Eike

Bauza, Antonio

Bavaro, Simona Lucia

Bavaro, Teodora 
Bayer, Peter

Bayón, Pau

Baz Etchebarne, Mariana

Bazylińska, Urszula

Bazzicalupi, Carla

Beata, Kalska-Szostko

Beaudoin, Daniel

Beaudry, Christopher

Bębenek, Ewa

Beberok, Artur

Bechthold, Andreas

Becker, Therese M.

Bedia, Carmen

Bedini, Emiliano

Bednar, Petr

Bedos-Belval, Florence

Beeton, Nick

Begley, David

Beharry, Kay D.

Behrends, Soenke

Beilby, Mary

Beis, Dimitris

Bekhit, Alaa El-Din A.

Bekiaris, Georgios

Bel'skaya, Lyudmila V.

Belancio, Victoria

Beld, Joris

Beletskaya, Irina P.

Belfrage, Anna Karin

Bell, Karen

Bell, Thomas W.

Bella, Federico

Bellés, José María

Bello, Claudia

Belmont, Philippe

Beloqui, Ana

Bełtowski, Jerzy

Ben Mahmoud, Lobna

Benati, Daniela

Bencsik, Timea

Bendas, Gerd

Benedec, Daniela

Benedetti, Michele

Beniah, Goliath

Benito, Antoni

Benito, José Manuel

Benito, Juan M.

Bennet, Andrew

Benseny-Cases, Nuria

Benso, Bruna

Benyoucef, Abdelghani

Benzeroual, Kenza E.
Berdonosov, Peter

Berenjian, Aydin

Berenyiova, Andrea

Beresford, Tom

Beretta, Giangiacomo

Beretta, Giovanni

Bergman, Jan

Berhow, Mark

Berlin, K. Darrell

Berlowska, Joanna

Bermudez, Marcel

Bernal, Patricia

Bernard, Marek K.

Bernardi, Luca

Bernardini, Giovanni

Bernini, Roberta

Bernstock, Joshua D.

Berrada, Houda

Berreau, Lisa M.

Berrocal, José Augusto

Berruyer, Romain

Berski, Wiktor

Bert, Nikolay

Berthelsen, Ragna

Berthold-Pluta, Anna

Berti, Federico

Bertin, Matthew J.

Berton, Paula

Bertran, Joan

Bertucci, Michael

Besalú, Emili

Bescond, Jocelyn

Besson, Thierry

Betancourt, Tania

Bethanis, Kostas

Bettuzzi, Saverio

Beutel, S

Beversdorf, David

Bevilacqua, Arturo

Beyeh, Ngong Kodiah

Bezirtzoglou, Eugénie

Bhang, Suk Ho

Bharadwaj, Vivek

Bhattacharya, Debswapna

Bhullar, Rajinder P.

Białoń, Marietta

Bian, Yongzhong

Bianchi, Antonio

Biancolillo, Alessandra

Bickhart, Derek M.

Biedermann, David

Bielenica, Anna 
Biernasiuk, Anna

Biesaga, Magdalena

Bijak, Michał

Bikiaris, Dimitrios

Bilewicz, Aleksander

Bilitewski, Ursula

Bill, Hawkins

Billard, Thierry

Bilyachenko, Alexey N.

Bimbo, Luis M.

Bingol, Kerem

Binzel, Daniel W.

Biondi, Elisa

Biot, Christophe

Birch, John

Birkemeyer, Claudia

Birringer, Marc

Bishop, Reid

Bisio, Alessandra

Bisio, Antonella

Bisoyi, Hari Krishna

Bisson, Jonathan

Bisti, Silvia

Bisz, Elwira

Black, David

Blackburn, Jessica

Blackwell, Barbara

Blair, Patricia

Blanco-López, María Carmen

Blando, Federica

Blanton, Cynthia

Blasiak, Janusz

Błaszczak, Wioletta

Blenda, Anna

Blériot, Yves

Bloom, Jesse

Bloor, Stephen

Bo', Cristian Del

Bo, Zhenyu

Board, Mary

Bobis, Otilia

Boccard, Julien

Bocchinfuso, Gianfranco

Boccia, Antonella Caterina

Bock, Thomas

Boddy, Christopher N.

Bode, Robert F.

Bodenstine, Thomas

Boeck, Lorenz R

Boeriu, Carmen

Boettcher, Shannon

Boga, Carla
Bogdan, Catalina

Bogdanowicz, Krzysztof Artur

Bøgwald, Jarl

Böhm, Volker

Böhmer, Frank

Bohn, Torsten

Boichuk, Sergei

Bojić, Mirza

Bokach, Nadezhda

Bollyky, Paul

Bolognini, Daniele

Bölükbas, Deniz

Bombard, Sophie

Bombarely, Aureliano

Bommagani, Shbanbabu

Bon, Volodymyr

Bonaccio, Marialaura

Bonamore, Alessandra

Bondon, Arnaud

Bonetta, Sara

Bongarzone, Salvatore

Bonifácio, Vasco

Bonikowski, Radosław

Bonnet, Pierre-Antoine

Bonomo, Robert A

Booth, Brian

Bora-Singhal, Namrata

Borawska-Dziadkiewicz, Justyna

Borbás, Anikó

Borcan, Florin

Bordenave-Juchereau, Stéphanie

Bordons, Albert

Borges, Anabela

Borges, Filipe

Borges, Keyller Bastos

Borges, Ricardo

Borghini, Andrea

Borin, Thaiz Ferraz

Bormashenko, Edward

Boros, Laszlo

Borota, Ana

Borovkov, Victor

Borst, Jan Willem

Boschi, Alessandra

Bose, Debojit

Bose, Sayantan

Boselli, Emanuele

Bosi, Sara

Bosland, Maarten C.

Bosse, Jens B.

Botelho, Monica C.

Botre, Francesco 
Bouaziz, Serge

Bouckaert, Julie

Boulton, Roger

Bouquillon, Sandrine

Bour, Petr

Bourguet-Kondracki, Marie-Lise

Boušová, Iva

Boutin, Jean

Boutin, Jean A.

Bowden, Gregory

Bowling, Heather

Boyarskiy, Vadim P.

Boysen, Reinhard

Bozonet, Stephanie M.

Božović, Mijat

Brábek, Jan

Bracci, Massimo

Brachet, Etienne

Brader, Günter

Bradshaw, Ben

Braga, Antonio

Braga, Guilherme S.

Braga, Susana

Braig, Simone

Brandstetter, Hans

Brandt, Curtis

Branza-Nichita, Norica

Branzoi, Florina

Braunecker, Wade

Bravo López, Susana B

Bravo, Jeronimo

Brčić Karačonji, Irena

Brebu, Mihai

Brecker, Lothar

Breier, Albert

Breinbauer, Rolf

Breitenfeld Granadeiro, Luiza

Brem, Jurgen

Bren, Urban

Brenna, Elisabetta

Brennan, Marian

Brenner, Robert

Brestic, Marian

Brettell, Laura E.

Brevern, Alexandre

Breza, Martin

Brichacek, Matthew

Briskey, David

Broberg, Anders

Broderick, Tom L.

Brooks, Peter

Brooks, Tracy A.
Bros, Matthias

Brouwers, Jos F.

Brown, Lindsay

Brown, Robert J.

Bruckner, Christian

Brudzynski, Katrina

Brullo, Chiara

Brun, Reto

Brune, Wolfram

Bruni, Giovanna

Bruning, John B.

Brunklaus, Gunther

Bruno, Olga

Brunschweiger, Andreas

Brust, Peter

Brustovetsky, Nikolay

Bryant, Matthew S.

Brycki, Bogumil

Brzeska, Joanna

Brzeziński, Marek

Brzozowski, Thomas

Bubb, Kristen J.

Bubici, Giovanni

Bubulya, Paula A.

Bučar, Dejan-Krešimir

Bucar, Franz

Buchet, Rene

Buchner, David

Buchowicz, Włodzimierz

Buchwald, Peter

Budnikova, Yulia

Budny, Marcin

Budryn, Grażyna

Budzak, Simon

Budzisz, Elżbieta

Bueno Cavanillas, Aurora

Bueno-Silva, Bruno

Bufo, Sabino Aurelio

Bugarin, Alejandro

Buisson, Didier

Bukin, Oleg

Bułakowska, Anita

Bulanin, Kirill

Bulgariu, Laura

Bullon, Pedro

Bultynck, Geert

Bun Ng, Tzi

Bunce, Richard A.

Bundgaard, Eva

Bundle, David R.

Bunik, Victoria

Bunt, Craig 
Bura-Nakić, Elvira

Burcul, Franko

Burger, Michael

Burghardt, Kyle

Burkard, Markus

Burns, Duncan Thorburn

Burpo, Fred J.

Burrola-Aguilar, Cristina

Bury, Wojciech

Busch, Hauke

Buschbeck, Marcus

Buß, Oliver

Bustamante, Paco

Bustos, Diego Martin

Butenschön, Holger

Butler, Mark

Butterworth, Peter J.

Buxton, Denis

Buzina, Walter

Byrne, Bernadette

Byrne, Hugh

Bystrowska, Beata

Bywater, Robert P.

Bzducha-Wróbel, Anna

Caballero, Julio

Caballero-Garcia, Beatriz

Cabral, Célia

Cabral, Horacio

Cabrales, Luis

Cabrele, Chiara

Cabrita, Ana Rita Jordão

Cacciola, Francesco

Cáceres González, Francisco F. Núñez De

Cadamuro, Massimiliano

Cádiz-Gurrea, María Luz

Caeiro, Maria

Caffrey, Patrick

Cafiero, Mauricio

Cahlíková, Lucie

Cahoon, Aubrey Bruce

Cai, Feng

Cai, Zhengxin

Caiazzo, Elisabetta

Cailliez, Fabien

Cairrão, Elisa

Cais-Sokolińska, Dorota

Calani, Luca

Calcabrini, Cinzia

Calcutt, Michael

Calderon, Christopher P.

Calderone, Christopher T.

Caligiuri, Isabella
Calingacion, Mariafe

Calokerinos, Antony C.

Calvano, Cosima Damiana

Calvete, Juan J.

Calvete, Mario

Camacho, M. Encarnación

Camacho-Corona, M. R.

Câmara, José Sousa

Camele, Ippolito

Camerlingo, Carlo

Caminade, Anne-Marie

Campaña, Araceli G.

Campana, Raffaella

Campanella, Claudia

Campardelli, Roberta

Campbell, Christopher

Campbell, Fiona M.

Campbell, Lee

Campestre, Cristina

Campos Da Rosa, Joaquim M

Campos-Martínez, J.

Campuzano, Susana

Canals, Francesc

Cancemi, Patrizia

Canetta, Elisabetta

Canini, Antonella

Cao, Xian

Cao, Zhe

Caparrotta, Stefania

Capasso, Raffaele

Capobianco, Amedeo

Capobianco, Giuseppe

Cappariello, Alfredo

Cappellini, Francesca

Capriati, Vito

Caputo, Gregory A.

Carbonell-Barrachina, Angel A.

Cárdenas, Soledad

Cardenia, Vladimiro

Cardia, Maria Cristina

Cardiano, Paola

Cardinale, Jens

Cardinale, Massimiliano

Cardinali, Alessandra

Cardoso, Hélia

Cardoso, Susana

Cardoso, Susana M.

Cardullo, Nunzio

Carella, Angelo M

Carillo, Petronia

Carlberg, Carsten

Carlisi, Daniela 
Carmona-Ribeiro, Ana M.

Carocho, Marcio

Carotti, Andrea

Carotti, Angelo

Carpene, Christian

Carqueijeiro, Inês

Carradori, Simone

Carrano, Carl J.

Carrasco, Sergio

Carrero, Jose Antonio

Carretti, Emiliano

Carta, Fabrizio

Carter, Nicola

Carter, Wayne

Carullo, Gabriele

Caruso, Giuseppe

Carvalho Pimenta, Daniel

Carvalho, Nakédia M.F.

Carvalho, Pedro

Casalino, Elisabetta

Casapullo, Agostino

Casas Ferreira, Ana María

Casassa, L. Federico

Casassa, Luis Federico

Cascioferro, Stella

Cascione, Mariafrancesca

Caselli, Alessandro

Caseri, Walter

Castaneda, Carlos H.

Castan-Laurell, Isabelle

Castellucci, Sonia

Castiglione, Franca

Castñeiras, Alfonso

Castro, Maria

Catani, Martina

Catanzano, Ovidio

Catizzone, Enrico

Cattaneo, Fabio

Catto, Marco

Catucci, Lucia

Cauli, Omar

Cava, Claudia

Cavaleiro, Carlos

Cefalas, A.C.

Celaya-Padilla, José M.

Celeiro, María

Celiński, Konrad

Cellamare, Saverio

Čenas, Narimantas

Cendrowski, Andrzej

Ceranowicz, Piotr

Cerchia, Laura
Černý, Jiřri

Cerón-Carrasco, José Pedro

Čeřovský, Václav

Cerra, Bruno

Ceruti, Stefania

Cesa, Stefania

Cha, Chaenyung

Chacón, Pablo

Chae, Heeyoung

Chai, Jeng-Da

Chai, Tian Feng

Chaimbault, Patrick

Chakraborty, Raja

Chamakura, Karthik

Chambers IV, Edgar

Chambon, Christophe

Chamorro-Posada, Pedro

Chamrád, Ivo

Chan, Ben C. L.

Chan, Yi-Tsu

Chan, Yu-Yi

Chandaka, Giri Kumar

Chandasana, Hardik

Chang, Che-Chien

Chang, Chia-Chen

Chang, Chien-Chung

Chang, Ching-yao

Chang, Dong Wook

Chang, Dong-Jo

Chang, Fang Rong

Chang, Geng-Ruei

Chang, Hai-Chou

Chang, Hao-Xun

Chang, Hsueh-Wei

Chang, Kee-Lung

Chang, Minsun

Chang, Sue-Joan

Chang, Te-Sheng

Chao, Chih-Hua

Chao, Louis Kuoping

Chao, Pi-Yu

Chapman, Eli

Charline, Kieffer

Chartier, Agnes

Chase, Mark W.

Chass, Gregory

Chatterjee, Jhinuk

Chatterjee, Ruchira

Chatterjee, Suman

Chatterley, Adam S

Chau, Chi-Fai

Chaung, Hso-Chi 
Chaves, Sílvia

Chaves-Lopez, Clemencia

Chavez, Ferman

Chazot, Paul L

Che, C.-T.

Che, Lixiao

Checa, Antonio

Checchetto, Riccardo

Chelbi, Sonia T.

Chemat, Farid

Chen, Chiachung

Chen, Chun-Chi

Chen, Chung-Yi

Chen, Chun-Jung

Chen, Guangping

Chen, Guoxun

Chen, Haixia

Chen, Haoyuan

Chen, Hongwei

Chen, Hsin-Chun

Chen, Hsing-Yin

Chen, Huaiqiong

Chen, Huixiong

Chen, Jen-Tsung

Chen, Jhy-Der

Chen, Jiahao

Chen, Jiann-Chu

Chen, Jianzhong

Chen, Jing-Hsien

Chen, Jun

Chen, Kew-Yu

Chen, Kun-Ming

Chen, Kuo-Yu

Chen, Liang-Yu

Chen, Lih-Geeng

Chen, Lin

Chen, Ming

Chen, Mingsheng

Chen, Min-Huey

Chen, Mohan

Chen, Po-Yuan

Chen, Qi

Chen, Qiao-Hong

Chen, Ruei-ming

Chen, Shih-Yu

Chen, Shiyu

Chen, Shuo-bin

Chen, Wenchun

Chen, Xi

Chen, Xiaoyan

Chen, Yu-Chih

Chen, Yu-Kuo
Chen, Yung-Chih

Chen, Yun-Wen

Chen, Zhao

Chen, Zhe

Chen, Zhihang

Chen, Zhijie

Chen, Zhonghua

Chen, Zhongning

Chénais, Benoit

Cheng, Bing-Ming

Cheng, Chih-Chia

Cheng, Gang

Cheng, Heung-Chin

Cheng, Hung-Chi

Cheng, Juei-Tang

Cheng, Jya-Wei

Cheng, Pei

Cheng, Qingqing

Cheng, Qing-Qing

Cheng, Sen-Sung

Cheng, Shouyun

Cheng, Wei-chieh

Cheng, Xingguo

Cheng, Zhihong

Chengwen, Sun

Cheon, Choong-ill

Cheong, Yong Hwa

Cheong, Yuen Ki

Cherkaoui-Malki, Mustapha

Chermette, Henry

Chern, Ming-Kai

Chetwynd, Andrew

Chi, Celestine N.

Chia, Win-Long

Chiang Hsieh, Lin-Han

Chiang, Anthony S.T.

Chiang, Hsiu-Mei

Chiang, Long Y.

Chiang, Meng-Tsan

Chiang, Ming-Hsi

Chiang, Tai-an

Chiang, Yi-Ting

Chien, Huan-Chieh

Chien, Shih-Chang

Chiessi, Ester

Chin, Young-Won

Chinchilla, Rafael

Chiodo, Fabrizio

Chiou, Chun-Tang

Chirumbolo, Salvatore

Chisholm, John D.

Chitchumroonchokchai, Chureeporn 
Chiu, Chi-cheng

Chiu, Tai-chia

Chiu, Tsai-Hsin

Chiulan, Ioana

Chiva-Blanch, Gemma

Chizzola, Remigius

Chladek, Grzegorz

Chlebek, Jakub

Chlichlia, Katerina

Chmiel, Tomasz

Chmielarz, Paweł

Chmielewska, Aleksandra

Chmielewska, Ewa

Chmurzyńska, Agata

Cho, Cheong-Weon

Cho, Dae Won

Cho, Eunae

Cho, Hea-Young

Cho, Michael

Cho, Nam-Joon

Cho, Seung-Sik

Cho, Sungeun

Cho, Sunghun

Cho, Sungyun

Chobot, Vladimir

Choi, Hyo-Jick

Choi, Jae Sue

Choi, Jae-Hong

Choi, Ki Choon

Choi, Min-Koo

Choi, Young Hae

Choi, Young Hee

Choi, Yung Hyun

Chojnacka, Anna

Cholewinski, Grzegorz

Chollet, Jean-François

Chong, Parkson

Choquesillo-Lazarte, Duane

Chou, Shih-Feng

Chou, Y.C.

Choudhary, Vivek

Chow, Aviva Shing Fung

Chow, Simon Kwoon-Ho

Chrissopoulou, Kiriaki

Christe, Karl O.

Christensen, Jørn B.

Christensen, Lars

Christians, Julian

Christians, Uwe

Christodoulou, Maria-Ioanna

Christoforides, Elias

Christopoulos, M. V.
Christova-Bagdassarian, Valentina

Chrobak, Elwira

Chrobok, Anna

Chruszcz, Maksymilian

Chu, Jiaxiang

$\mathrm{Chu}$, Shidong

Chu, Yen-Wei

Chua, Beelee

Chuck-Hernández, Cristina

Chuffa, Luiz Gustavo

Chulhai, Dhabih V

Chun, Heung Jae

Chung, Byung Min

Chung, Hyun-Jung

Chung, Jing-Gung

Chung, Ying-Chien

Chunsuo, Yao

Chuyen, Hoang V.

Chyau, Charng-Cherng

Ciaccheri, Leonardo

Ciancaleoni, Gianluca

Cianciosi, Danila

Ciccone, Marco Matteo

Çiçek, Serhat Sezai

Cicero, Arrigo

Cicero, Arrigo Francesco Giuseppe

Cichero, Elena

Cichorek, Miroslawa

Ciecholewski, Marcin

Cieniewski-Bernard, Caroline

Cieplik, Fabian

Ciereszko, Iwona

Cież, Dariusz

Cigáň, Marek

Cihacek, Larry

Cilla, Antonio

Cimmino, Giovanni

Cimpoiu, Claudia

Cinelli, Patrizia

Cinteza, Ludmila Otilia

Ciobica, Alin

Ciofani, Gianni

Ciofi-Baffoni, Simone

Cione, Erika

Cirri, Damiano

Cirrincione, Girolamo

Cisowsky, Jaroslaw

Citterio, Barbara

Ciulu, Marco

Čizmić, Mirta

Claridge, Jolyon $\mathrm{K}$

Clark, Andrew 
Clark, Timothy

Clarke, Ron

Claus, Harald

Clausen, Rasmus Prætorius

Clement, Cristina

Clericuzio, Marco

Close, David M.

Cobo, Martha

Coburn, Jeannine

Coccheri, Sergio

Coccia, Gianluca

Cocetta, Giacomo

Cochis, Andrea

Cochran, Blake

Cochrane, Stephen

Codacci-Pisanelli, Giovanni

Codee, Jeroen

Coelho, Carolina

Coen, De Graaf

Coey, J.M.D.

Coldea, Teodora

Cole, Kathryn E.

Colecchia, David

Coll, Josep

Collier, Jason

Collina, Simona

Collins, Stuart G.

Cologna, Stephanie

Colombo, Raffaella

Colonna, Giovanni

Colquhoun, Thomas A.

Coluccia, Mauro

Comai, Stefano

Comito, Robert J

Commisso, Mauro

Compostella, Federica

Conceição, Katia

Concepción Heydorn, Patricia

Concilio, Simona

Connolly, Joseph

Constantin, Lucian

Constantinescu, Stefan N.

Constantinou-Kokotou, Violetta

Conte, Giuseppe

Conte, Lanfranco

Conti, Barbara

Contiero, Jonas

Contino, Marialessandra

Copolovici, Dana Maria

Corato, Riccardo Di

Corazza, Marcos Lúcio

Corazzin, Mirco
Corbett, Charlotte

Cordaro, Marika

Cordeiro, Rodrigo Maghdissian

Corke, Harold

Cornejo, Javiera

Coronas Ceresuela, Joaquín

Corradini, Danilo

Corradini, Roberto

Corradini, Valdis

Corrales-Garcia, Joel

Correa, Arkaitz

Correia, Ana Cristina

Correia, Ilidio

Corrigan, Damion

Corsaro, Carmelo

Cortés Castell, Ernesto

Cosco, Donato

Costa Lima, Sofia

Costa Moutinho, Fabíola

Costa, Blaise Mathias

Costa, Geison

Costa, Maria-margarida R. R.

Costa, Paulo Jorge

Costa, Pedro

Costa, Rosaria

Costa, Stefania

Costantino, Luca

Couce, María D.

Couderc, François

Coulon, Stéphane

Coutinho, Paulo José Gomes

Cowen, Bryan J.

Coy-Barrera, Ericsson

Coyne, Mark

Cozzolino, Anthony

Cozzolino, Daniel

Cragg, Peter

Crascì, Lucia

Cravero, Maria Carla

Crawford, Philip W.

Creamer, Rebecca

Crestini, Claudia

Creus, Marc

Crișan, Gianina

Crisan, Ovidiu

Crnkovic, Ana

Croce, Anna Cleta

Crocket, Kirsty

Croset, Martine

Crowley, James

Crowley, Peter

Crucho, Carina 
Crucho, Carina Isabel Correia

Cruz, Adriano

Cruz-Sosa, F.

Csámpai, Antal

Cuadrado, Angeles

Cucciniello, Raffaele

Cueto, Mercedes

Cui, Tiantian

Cui, Xin

Cui, Yi

Cui, Zhibin

Cullen, Carlie

Culot, Maxime

Cunha, Luís Miguel

Cupellini, Lorenzo

Curti, Claudio

Curti, Daniela

Custódio, Danilo

Cutrignelli, Annalisa

Cycoń, Mariusz

Czaplewski, Cezary

Czarnocki, Zbigniew

Czechowski, Tomasz

Czekelius, Constantin

Czerniewicz, Paweł

Czerwinska, Monika

Czjzek, Mirjam

Czopka, Tim

Czuba, Zenon

Czyżowska, Agata

D' Alessandro, Angelo

D'Anna, Francesca

Da Motta, M.

Da Silva, Mateus Webba

Da Silva, Monique Gabriella Angelo

D'Abrosca, Brigida

D'accolti, Lucia

Dacheux, Jean-Louis

Dachineni, Rakesh

Dagorne, Samuel

D'Agostino, Nunzio

Dai, Yang D

Dai, Yumin

Dai, Zhigao

Dal Ben, Matteo

Dal Piaz, Fabrizio

Dallacosta, Lorenza

Dall'Acqua, Stefano

Dall'Asta, Chiara

Dallavalle, Sabrina

Dallemagne, Patrick

Dalmoro, Annalisa
Dalpozzo, Renato

Daly, Norelle

Damalanka, Vishnu

Damiani, Daniela

Damjanović, Marko

Damjanovski, Sashko

Damkaci, Fehmi

Da'na, Enshirah

Danciu, Corina

Dandekar, Thomas

Danezis, Georgios

Dang, Jing-Shuang

Dangles, Olivier

Danielak, Dorota

Daniele, Aurora

Daniellou, Richard

Danielson, Neil

Daniels-Wells, Tracy R.

Dansette, Patrick M.

Dante, Silvia

Darafsheh, Arash

D'Argenio, Valeria

Darvesh, Sultan

Das, Anindita

Dasgupta, Kasturi

Dasgupta, Santanu

Dasgupta, Subham

Dash, Alekha K.

Dash, Biraja

Dash, Ranjeet

Datta, Kausik

Daugrois, Jean-Heinrich

D'Auria, Enza

Davari Esfahani, M. Mahdi

David-Cordonnier, Marie-hélène

Davies, Christopher

Davinelli, Sergio

Davis, Keith R.

Davis, Mark

Dawid, Corinna

Dawn, Arnab

De Almeida Coelho De Sousa, Maria João

De Almeida, Andreia

De Alvarenga, Elson Santiago

De Amici, Marco

De Andrade, Sara Adrián López

De Aza, Piedad N.

De Barros Fernandes, Pedro

De Bellis, Luigi

De Berardis, Domenico

De Bock, Marijke

De Caro, Viviana 
De Carvalho Santos Ebinuma, Valéria

De Castro, Sonia

De Deurwaerdère, Philippe

De Diego, Marta

De Feo, Vincenzo

De Giudici, Giovanni

De Gonzalo, Gonzalo

De Jesus, Ernesto

De Jonghe, Steven

De Kimpe, Norbert

De La Garza, Mireya

De La Lande, Aurélien

De La Torre, Maria Del Carmen

De Lacey, Antonio Lopez

De Lago, Eva

De Leo, Marinella

De Marco, Rossella

De Masi, Luigi

De Matteis, Valeria

De Mendonça, Dina I. M. D.

De Mieri, Maria

De Oliveira Carvalho, Carla Roberta

De Oliveira, Kleber

De Paula Venancio, Vinicius

De Rosa, Maria Cristina

De Simeis, Davide

De Sousa, Frederico B.

De Sousa, Maria Emilia

De Tullio, M.C.

De Vendittis, Emmanuele

De Visser, Samuel

De Vleeschouwer, Freija

De Vries, Carlie

De Wit, Maryna

De With, Gijsbertus

Dealy, Caroline N.

Dean, Lisa

Dean, Lisa Oehrl

Deb, Subrata

Debnath, Asim Kumar

Decristoforo, Clemens

DeDiego, Marta L.

Degano, Ilaria

Degennaro, Leonardo

Degiacomi, Matteo T.

Degola, Francesca

Dehaen, Wim

Dehzangi, Abdollah

Deineka, Victor

Dejam, Morteza

Del Giudice, Rita

Del Río Celestino, Mercedes
Del Rio, Daniele

Delannoy, Etienne

Delannoy, Philippe

Delaude, Lionel

Delbecq, Frédéric

Delfin, Dawn A.

Della Ca', Nicola

Delogu, Giovanna

Delorme, Vincent

Delville, Marie-Hélène

Dembska, Anna Renata

Demchenko, Alexei V.

Demir, Fatih

Demonacos, Constantinos

Demopoulos, Vassilis J.

Den, Walter

Denat, Franck

Deng, Jianjun

Deng, Jing

Deng, Qing

Deng, Ruixia

Deng, Xiaoran

Denis, Sereno

Deo, Permal

Deokar, Hemantkumar

Depeint, Flore

Deplazes, Evelyne

Deplus, Rachel

DeRosa, Christopher

Desai, Jigar

Desai, Umesh R.

Desando, Giovanna

Deshmukh, Rupesh

Deshpande, Rahul

Deshpande, Shayu

Dessolin, Jean

D'Este, Matteo

Detsi, Anastasia

Deuss, Peter

Devadas, Krishnakumar

Devasurendra, Amila

Dey, Gangotri

DeYonker, Nathan J.

Dhakal, Dipesh

Dhakshinamoorthy, Amarajothi

Dhanasekaran, Danny N.

D'hulst, Christophe

Di Bella, Santo

Di Biase, Stefano

Di Buduo, Christian

Di Bussolo, Valeria

Di Carlo, Marta 
Di Donato, Marzia

Di Gioia, Maria Luisa

Di Liegro, Italia

Di Luca, Mariagrazia

Di Luccia, Aldo

Di Marino, Daniele

Di Mauro, Maria Domenica

Di Natale, Concetta

Di Pasqua, Anthony J.

Di Pierro, Prospero

Di Sotto, Antonella

Di Stefano, Antonio

Di Tommaso, Devis

Diaba, Faïza

Diamond, Gill

Diana Camelia, Nuţă

Diana, Rosita

Dias, Rolando CS

Díaz, Cristina

Diaz, Gaspar

Díaz, José

Dichiara, Anthony

Dickert, Franz L.

Diculescu, Victor Constantin

Die, Jose

Diekman, Alan

Díez, David

Dijkhuizen, Lubbert

Dijkstra, Bauke W.

Dilonardo, Elena

Dimakis, Nicholas

Dimitrakopoulos, Georgios

Dimitroff, Charles J.

Dina, Nicoleta Elena

Dinadayalane, Tandabany

Ding, Baoqing

Ding, Chunbang

Ding, Jianxun

Ding, Ling

Ding, Wei Dan

Ding, Zhaoyang

Dinica, Rodica Mihaela

Dinis, Maria Alzira Pimenta

Diniz, Carmen

Diniz, Paulo Henrique Gonçalves Dias

Dinu-Pîrvu, Cristina Elena

Dionisio, Giuseppe

Diretto, Gianfranco

Dirsch, Verena

Disdier, Clemence

Dixit, Mudit

Dixon, David
Dixon, David A.

Dmitrenok, Pavel S.

Do Carmo, Sergio J. C.

Dobrovolskaia, Marina

Dobrzynski, Piotr

Doda, Sai Reddy

Doddapaneni, Ravi

Dodero, Veronica

Doerksen, John Robert

Döhler, Diana

Doi, Hisashi

Doi, Takayuki

Dolashka, Pavlina

Doležal, Karel

Dolga, Amalia

Dolzhenko, Anton

Domine, Marcelo

Domínguez-Álvarez, Enrique

Dominikowska, Justyna

Donahue, Matthew

Donato, Rosario

Dong, Shihui

Dong, Yibo

Dong, Yuesheng

Donnarumma, Giovanna

Donno, Dario

Doolittle, Russell F

Doran, Todd

Doria, Filippo

Doriano Bianco, Armando

Dorin, Julia

Dorotkiewicz-Jach, Agata

Dos Santos Niculau, Edenilson

Dos Santos, Demetrio

Dos Santos, José C.S.

Dos Santos, Reinaldo Sousa

Doseff, Andrea

Dosoky, Noura S.

Dossena, Silvia

Douris, Vassilis

Dovinova, Ima

Doyle, Siamsa

Dračínský, Martin

Dragomir, Mihnea

Drasar, Pavel

Drewniak, Sabina

Drozdov, Aleksey D.

Drummond, Rebecca

Du, Deguo

Du, Fanfan

Du, Guodong

Du, Shu Shan 
$\mathrm{Du}$, Yongle

Duarte Campos, Daniela Filipa

Duarte, Andreia

Duarte, Maria Cristina

Duarte-Carvajalino, Julio M.

Dubey, Ramin

Dubielecka, Patrycja M.

Dudas, Jozsef

Dudnikov, Alexander Ju.

Dueñas-Patón, Montserrat

Düfer, Martina

Dufossé, Laurent

Duggan, Brendan $\mathrm{M}$.

Duh, Pin-Der

Duma, Luminita

Dumas, Françoise

Dumur, Frédéric

Dunckley, Travis

Dundas, Ian

Duong, Haiminh

Duong-Quy, Sy

Dupont, Laurent

Durães, Luisa

Durand, Jean-Olivier

Durán-Peña, María Jesús

Durazzo, Alessandra

Durek, Thomas

Durgo, Ksenija

Dutartre, Patrick

Dutoi, Anthony D.

Dutta, Arijit

Dutta, Biswanath

Dutta, Debarun

Dutta, Palash

Duval, Raphaël E.

Duvall, Melvin

Dvir, Hay

Dvoyashkin, Muslim

Dwivedi, Chandrahar

Dworak, Andrzej

Dyguda-Kazimierowicz, Edyta

Dzidic, Alen

Dziedzic, Arkadiusz

Dziegielewska, Barbara

Dziewit, Lukasz

Dziki, Dariusz

Dzikovski, Boris

Dzimitrowicz, Anna

Dziuba, Dmytro

Dzolic, Zoran

Dzuba, Sergei Andreevich

Ebani, Velentina
Ebrahim, Weaam

Ebrahimi, Aida

Echevarria, Aurea

Eckl, Peter

Edafiogho, Ivan

Edalati, Kaveh

Eder, Iris E.

Eder, Matthias

Edin, Nina Jeppesen

Edlund, Ulrica

Edmondo, Battista

Edreira, Martin M.

Edström, Daniel

Edwards, Matt

Efimov, Alexander

Efremenko, Elena

Egami, Hiromichi

Egerszegi, István

Eggeling, Lothar

Egodawatte, Shani

Eguiraun, Harkaitz

Ehlers, Peter

Ehlert, Claudia

Ehm, Christian

Ehrich, Marion

Ehrlich, Garth

Eid, Nabil

Eiroa, José Luis

Eisenreich, Wolfgang

Ejfler, Jolanta

Ejidike, Ikechukwu P.

Ekielski, Adam

Ekiert, Halina

Eklund, Patrik C.

Elaissari, Abdelhamid

El-Bacha, Tatiana

Eldeeb, Mohamed

Eleftheriou, Eleftherios P.

Elemans, J.A.A.W.

Elghobashi-Meinhardt, Nadia

Elkordy, Amal

Eller, Fred

Elleuche, Skander

Elli, Stefano

Elmann, Anat

El-Mashtoly, Samir F.

El-Safty, Sherif A.

El-Seedi, Hesham R.

Elshafie, Hazem S.

Elshahawi, Sherif

Elsinghorst, Paul W.

Emerson, Joseph 
Emery, Neil

Empl, Michael

Enchev, Venelin

Endo, Takeshi

Endres, Kristina

Engel, Matthias

Engel, Paul C.

Engle, Jonathan

Enguita, Francisco J.

Enkvist, Erki

Ennas, Guido

Erdem, Emre

Ersan, Sevcan

Esadze, Alexandre

Escalante, Carlos

Escalante, Jaime

Escames, Germaine

Escario, José

Escorihuela, Jorge

Eslami, Hossein

Espada, Jesús

Espinosa-Diez, Cristina

Esposito, Emilio Xavier

Espuelas, Socorro

Esque, Jérémy

Esquinazi, Pablo

Estelrich, Joan

Esteve-Romero, Josep

Esteves, Ana Cristina

Estévez Cabanas, Ramón J.

Etacheri, Vinodkumar

Eu, Peter

Evans, Joseph L.

Eves-van Den Akker, Sebastian

Evtugyn, Gennady

Ezekiel, Uthayashanker

Ezquerra-Brauer, Josafat-Marina

F. Rasulev, Bakhtiyor

Fabiani, Roberto

Fabiano, Eduardo

Facão, Jorge

Faggio, Caterina

Failla, Cristina M.

Fairlamb, Alan

Faldyna, Martin

Falentin-Daudré, Céline

Fales, Andrew

Fallon, Thomas

Falqué, Elena

Fan, Chenguang

Fan, Weizheng

Fan, Xiulin
Fan, Yunchang

Fan, Zhijin

Fanali, Chiara

Fanfrlík, Jindřich

Fang, Marong

Fang, Mingxi

Fang, $X i$

Fang, Xiaolan

Fang, Yi

Fanizzi, Francesco Paolo

Fantacuzzi, Marialuigia

Farag, Mohamed

Farantos, Stavros C.

Farcas, Anca Corina

Fardel, Olivier

Fare, Silvia

Farhad, Ali

Farhat, Grace

Faria, Ana

Farjon, Jonathan

Farkaš, Pavol

Farmer, Patrick

Farrell, Robert

Farrugia, Brooke

Fasinu, Pius S.

Fateixa, Sara Isabel Augusto

Fatouro, Dimitris

Faure, Sébastien

Faustino, M. Amparo F.

Favas, Paulo J.C.

Favero, Gaia

Favier, Isabelle

Favre-Réguillon, Alain

Feas, Xesus

Fedorov, Alexey

Feiner, Zachary S.

Feldblyum, Jeremy I.

Felgueiras, Helena

Feliciano, Gustavo Troiano

Feliu, Lidia

Fenaille, Francois

Feng, Chia-Hsien

Feng, Zhihui

Feng, Zhiwei

Fenyvesi, Ferenc

Feregrino-Pérez, Ana A.

Ferguson, Bradley

Ferguson, Matthew

Fernadez-Bolanos, José G.

Fernandes, Eduarda

Fernandes, $\mathrm{P}$.

Fernández, Belén 
Fernández, Gustavo

Fernandez-Botran, Rafael

Fernández-Lafuente, Roberto

Fernandez-Muino, Miguel

Fernández-Zertuche, Mario

Ferraboschi, Patrizia

Ferrante, Antonio

Ferrante, Claudio

Ferreira, Jorge

Ferreira, Leonardo G.

Ferreira, Marcelo

Ferreira, Paula M.

Ferreri, Carla

Ferretti, Elisabetta

Ferretti, Valeria

Ferris, William

Ferruz-Capapey, Noelia

Fia, Giovanna

Figadere, Bruno

Figueira, Rita Bacelar

Figueiredo, Ana Cristina S

Figueroa-López, Alejandro Miguel

Fijan, Sabina

Filannino, Pasquale

Filarowski, Aleksander

Filek, Maria

Filho, Edson C. Da Silva

Filipova, Inese

Filippov, Andrei

Fill, Taícia Pacheco

Filosto, Massimiliano

Fina, Emanuela

Fine, James Burke

Finšgar, Matjaž

Fiorati, Andrea

Fioravanti, Stefania

Fiorino, Ferdinando

Firlak, Melike

Fisher, Jed F.

Fisslthaler, Beate

Fleischer, Ivana

Fletcher, Nicola F.

Fletcher, Steven

Fleurat-Lessard, Paul

Flieger, Jolanta

Floresta, Giuseppe

Fodor, Csaba

Fodor, Marietta

Fogolari, Federico

Fologea, Daniel

Fong, Bertram Y.

Fontaine, Thierry
Fontanesi, Claudio

Fontàs, Clàudia

Fontés, Michel

Foray, Genevieve

Forbes, Tamara

Formela, Krzysztof

Formisano, Carmen

Forssell-Aronsson, Eva

Fortes, Ana Margarida

Forti, Luca

Fossa, Paola

Foster, Tim

Fotopoulos, Vasileios

Foubelo, Francisco

Fouillaud, Mireille

Fouillen, Laetitia

Fourmentin, Sophie

Fourmigue, Marc

Fourtounas, Costas

Fousteris, Manolis

Fox, Jay W.

Fraaije, Marco

Fraile, Alberto

Franca, Eduardo F

Francesco, Negro

Francesco, Stellato

Francis, Ashwanth Christopher

Franco, Albina Cristina Ribeiro

Franco, Carlos M.

Franco, Lourdes

Franco, Rodrigo

Frank, Craig L.

Frank, Michael

Frank, Saša

Frank, Schweizer

Franko, Andras

Frankowski, Marcin

Franus, Wojciech

Franz, Chlodwig

Franzoi, Marco

Franzyk, Henrik

Fratianni, Florinda

Frau, Juan

Frazzi, Raffaele

Frederix, P.W.J.M. (Pim)

Freire, Juan

Freires, Irlan Almeida

Freitas, Ana

Freund, Nadja

Frezza, Claudio

Friel, Claire

Frielinghaus, Henrich 
Frings, Patrick

Frkanec, Ruža

Frochot, Celine

Froeyen, Matheus

Froeyen, Mathy

Fröhlich, Eleonore

Froldi, Guglielmina

Frolov, Andrej

Frolova, Liliya

Frongia, Angelo

Frontera, Antonio

Frosini, Maria

$\mathrm{Fu}$, Junjie

$\mathrm{Fu}, \mathrm{Li}$

$\mathrm{Fu}$, Peng

Fu, Shih-Feng

$\mathrm{Fu}$, Xueyan

$\mathrm{Fu}, \mathrm{Yu}$

Fuglsang, Anja Thoe

Fuhrmann, Gregor

Fujie, Tomoya

Fujihara, Tetsuaki

Fujii, Hideaki

Fujii, Kazuko

Fujii, Satoshi

Fujimori, Ko

Fujimoto, Kenzo

Fujimura, Yoshinori

Fujisawa, Tomotsumi

Fujita, Masaki

Fujita, Morifumi

Fujiwara, Hideki

Fujiwara, Ryoichi

Fukuda, Mitsuru

Fukuhara, Toshiyuki

Fukunaga, Kenji

Fukushi, Keiichi

Fukushima, Tomohiro

Fuller, Amelia

Fumagalli, Laura

Fumanal, Maria

Funabiki, Kazumasa

Fuoco, Tiziana

Furneri, Pio Maria

Furness, James

Fürnsinn, Clemens

Furtula, Boris

Fuse, Shinichiro

Fuso, Francesco

Futai, Masamitsu

G Gamage, Dilani

G. Guirao, Juan Luis
Gabdulkhakov, Azat

Gábor, Vasas

Gaczynska, Maria

Gafencu, Anca

Gaffet, Eric

Gagat, Maciej

Găină, Luiza Ioana

Gakiere, Bertrand

Galabov, Angel

Galan, Carmen

Galan, Javier Sanchez

Galán, Miguel Ladero

Galanis, Alexis

Galasiti Kankanamalage, Anushka

Galat, Andrzej

Galdeano, Carles

Galeazzi, Roberta

Galic, Ante

Gálik, Branislav

Galindo, Antonio

Galindo-Murillo, Rodrigo

Gall Troselj, Koraljka

Gallardo, Mercedes

Gallemí, Marçal

Galletti Raspolli, Anna Maria

Galuska, Sebastian P.

Galván-D' Alessandro, Leandro

Galvez Llompart, Maria

Gambino, Giorgio

Gambuti, Angelita

Gamet-Payrastre, Laurence

Gan, Renyou

Gan, Ren-You

Gan, Samuel

Gan, Yong X.

Gandhi, Neha S.

Ganesan, A.

Ganesan, Palanivel

Gangaraju, Rajashekhar

Gangula, Pandu

Gannett, Peter M.

Gantenbein, Benjamin

Gao, Jianzhao

Gao, Jie

Gao, Peiyuan

Gao, Wenyang

Gao, Xiaolong

Gao, Xiaoyan

Gao, Zhe

Garad, Sudhakar

Garaj, Vladimír

Garami, Andras 
Garbowska, Monika

García De Frutos, Pablo

Garcia Estévez, Ignacio

García López, Víctor

Garcia Vaquero, Marco

García-Beltrán, Olimpo

Garcia-Bosch, Isaac

García-Fresnadillo, David

García-Garrido, Sergio E.

Garcia-Gimenez, Dolores

García-Iriepa, Cristina

Garcia-Jares, Carmen

García-Moreno, Valme

Garcia-Pardo, Javier

Garcia-Segura, Sergi

Garcia-Sosa, Alfonso T.

García-Villalón, Angel Luis

Gard, Paul

Garg, Akhil

Garinis, George

Garno, Jayne

Garo, Eliane

Garrote, Gil

Gartia, Manas

Gasbarri, Carla

Gase, Klaus

Gašić, Uroš M.

Gasparrini, Massimiliano

Gaston, Kirk

Gatti, Antonietta

Gauden, Piotr A.

Gaul, David

Gauld, James W.

Gaumann, Andreas

Gavara, Laurent

Gavara, Rafael

Gawlik-Dziki, Urszula

Gawron-Skarbek, Anna

Gdaniec, Zofia

Gdula-Argasinska, Joanna

Ge, Dongxia

Ge, Liya

Ge, Xiaodong

Ge, Yufeng

Gebicki, Janusz

Geissler, Daniel

Geletii, Yurii

Gellerman, Gary

Gelli, Angie

Gelpi, Josep Lluis

Gemma, Sandra

Gemmill, Trent
Geng, Anli

Geng, Feng

Geng, Junlong

Genisheva, Zlatina

Genova, Tullio

Genta-Jouve, Grégory

Gentile, Marialuisa

Gentile, Piergiorgio

Gentili, Alessandra

Georghiou, Paris

Georgiades, Savvas

Georgiev, Anton

Georgiev, Georgi

Georgiev, Vasil

Georgieva, Radostina

Georgopanos, Prokopios

Geraghty, Patrick

Gerardi, Carmela

Gérardin-Charbonnier, Christine

Gerasimova, Yulia V.

Gerber, Leonard E.

Gerdol, Marco

Gereke, Thomas

Gerke, Jörg

Gerken, Michael

Geronès, Ferran Feixas

Gerothanassis, Ioannis P.

Gersen, Henkjan

Gervasi, Teresa

Gervasoni, Jacopo

Gessner, Guido

Ghandi, Khashayar

Ghani Zadeh, Hossein

Ghanotakis, Demetrios F

Ghavami, Mohammad

Gheni, Ahmed A.

Gheorghe, Marian

Ghiasvand, Alireza

Ghica, Mihaela-Violeta

Ghislat, Ghita

Ghosh, Santosh

Ghosh, Subrata

Giacomelli, Chiara

Giacometti, Jasminka

Giacomini, Daria

Giacomino, Agnese

Giacosa, Simone

Giampieri, Francesca

Giannakas, Aris

Giannakoudakis, Dimitrios

Giannakouros, Thomas

Giannakourou, Maria C. 
Giannenas, Ilias

Giarratana, Filippo

Giebułtowicz, Joanna

Gieling, Roben G.

Gierlinger, Notburga

Gierszewska, Magdalena

Giesbertz, Pieter J.

Gigoux, Véronique

Gikas, Euaggelos

Gil, Barbara

Gil, Minchan

Gilabert-Oriol, Roger

Giles, Wayne R.

Gilheany, Declan G.

Gill, Harsimran K.

Gilli, Francesca

Gil-Ramirez, Guzman

Gilroyed, Brandon

Ginés Benito, Martínez Hernández

Ginex, Tiziana

Gioiello, Antimo

Giomi, Donatella

Giordano, Daniela

Giordano, Luana

Giorgi, Giorgio

Giosafatto, Concetta Valeria Lucia

Giovannucci, David R.

Girelli, Chiara Roberta

Gismondi, Angelo

Giuffrè, Angelo Maria

Giuntini, Francesca

Giurg, Mirosław

Giuseppina, Andreotti

Głąbska, Dominika

Gładkowski, Witold

Gladyshev, Michail

Glaeser, Jessie

Glasovac, Zoran

Globisch, Daniel

Glover, Stephen A.

Gmeiner, William H.

Gmurek, Marta

Gnanamani, Muthu Kumaran

Gnavi, Giorgio

Gniewosz, Małgorzata

Gnyba, Marcin

Gobeaux, Frédéric

Gobis, Katarzyna

Godlewska-Żyłkiewicz, Beata

Godlewski, Szymon

Goel, Peeyush

Goettig, Peter
Goffart, Steffi

Gogulamudi, Venkateswara Reddy

Gohda, Eiichi

Gok, Ozgul

Golczak, Marcin

Gold, Ben

Golding, Jon

Golkowski, Czeslaw

Goltz, Douglas M.

Golubkina, Nadezda Aleksandrovna

Gomes, Maria S.

Gomes, Nelson

Gomes, Nelson G.M.

Gómez De Cedrón, Marta

Gómez García, Carlos J.

Gómez, Ana María

Gómez-Merino, Ana Isabel

Gómez-Pastor, Rocío

Gomez-Plaza, Encarna

Gómez-Ruiz, Santiago

Gomha, Sobhi

Gonçalves, Fernando J.

Gonçalves, Lidia M D

Gonçalves, M. Sameiro T.

Gondi, Christopher S

Gonec, Tomas

Goněc, Tomáš

Gong, Xingchu

Gontier, Eric

Gonzales, Cara B.

González Cortés, Araceli

González Domínguez, Raúl

González, Florenci V.

Gonzalez, Juan Miguel

González, María Eugenia

González-García, Jorge

González-Laredo, Rubén Francisco

González-Maeso, Javier

González-Muñiz, Rosario

González-Sánchez, M. I.

González-Stuart, Armando

González-Vallinas, Margarita

González-Vergara, Enrique

Gopalan, Sai Anand

Gordillo Arrobas, Belén

Gordon, Michael H.

Górecki, Marcin

Goreham, Renee

Gorgoulis, Vassilis

Gorham, Edward

Gori, Antonella

Gorman, Greg 
Goroncy, Alexander

Gorrell, Mark

Górska, Sabina

Gorska-Ponikowska, Magdalena

Gorte, Raymond J.

Gościańska, Joanna

Gosetti, Fabio

Gossage, R. A.

Goswami, Subhadip

Goszczyński, Tomasz

Goto, Masuo

Gotor Fernandez, Vicente

Gouany, Frady

Goudarzi, Maryam

Goulas, Vlasios

Goupil, Brad A

Goze, Christine

Gozzelino, Raffaella

Gozzo, Franco

Grabacka, Maja

Grabarczyk, Małgorzata

Grabulosa, Arnald

Gradinaru, Andrei Cristian

Graiff, Claudia

Grajek, Henryk

Granados-Principal, Sergio

Granchi, Carlotta

Grande, Fedora

Grande, Rossella

Grandjean, Cyrille

Granica, Sebastian

Grant, George

Grant, William

Granzhan, Anton

Grasselli, Elena

Grassmann, André

Graton, Jérôme

Gray, Christopher A.

Grazioso, Giovanni

Green, Charlotte

Green, Ivan Robert

Greenberg, Arthur

Greenhalgh, David

Gregáň, Juraj

Gregory, Stephen

Greim, Helmut

Grellier, Philippe

Grembecka, Małgorzata

Grider, Arthur

Griesbeck, Axel

Griesser, Michaela

Griffiths, Peter
Griga, Miroslav

Grijalvo, Santiago

Grisanti, Luca

Grisoni, Francesca

Grivel, Jean-Claude

Grkovic, Tanja

Gross, Aaron

Grossi, Giancarlo

Grossi, Marco

Grossini, Elena

Grossman, Jerrold W.

Grosso, Giuseppe

Gruber, Christian

Gruhlke, Martin

Grunig, Gabriele

Grützmacher, Hansjörg

Grynkiewicz, Grzegorz

Grzegorczyk-Karolak, Izabela

Grzybowska, Ewa

$\mathrm{Gu}$, Mengmeng

$\mathrm{Gu}$, Zhen

Guadagno, Carmela

Guamis, Buenaventura

Guarcello, Rosa

Guarino, Carmine

Gubin, Denis

Gudbrandsen, Oddrun A.

Guerrini, Alessandra

Guha, Prajna P.

Guibal, Eric

Guido, Luis F.

Guillon, Christophe

Guiot, Caterina

Gullón, Beatriz

Gumieniczek, Anna

Gumienna, Małgorzata

Gündisch, Daniela

Gündoğ, Yücesan

Gunia-Krzyżak, Agnieszka

Guo, Fenghai

Guo, Pu

Guo, Shaoyun

Guo, Xiaofeng

Gupta, Kajal

Gupta, Sanjay

Gupta, Sheeba Varghese

Gupta, Vivek

Gupta-Rossi, Neetu

Gurgul, Artur

Gurikov, Pavel

Gursoy, Gamze

Gusev, Alexander 
Gushina, Irina

Gutiérrez-Gallego, Ricardo

Gutiérrez-Uribe, Janet

Gütschow, Michael

Guvench, Olgun

Guzik, Urszula

Guzman-Puyol, Susana

Guzow-Krzemińska, Beata

H. Moreno, Paulo Roberto

Habash, Marc

Habata, Yoichi

Habtemariam, Solomon

Hachemi, Imane

Hackauf, Bernd

Hackett, Mark

Hackmann, Timothy

Hadaruga, Daniel

Hadjikakou, Sotiris

Hadjipavlou-Litina, Dimitra

Haenen, Guido

Hafuka, Akira

Hager, Martin D.

Haidar, Samer

Haider, Norbert

Haider, Shozeb

Hains, David

Halder, Avik

Ham, Kyung-Sik

Hamaguchi, Masahide

Hamman, Joias

Hammann, Simon

Hammer, Katherine

Hammerl, Jens Andre

Hammerschmidt, Ray

Han, Byung Woo

Han, Jaehong

Han, Jianlin

Han, Jong Won

Han, Kewen

Han, Mingyuan

Han, Qifeng

Han, Quan-Bin

Han, Sung Nim

Han, Weiwei

Han, Won-Sik

Han, Yifan

Handzlik, Jadwiga

Haneklaus, Moritz

Hanganu, Daniela

Hanhineva, Kati

Haniu, Hisao

Hannafon, Bethany
Hano, Christophe

Hanschen, Franziska

Hansen, Espen

Hansen, Niels

Hanus-Fajerska, Ewa

Hao, Jijun

Happel, Austin

Harada, Mamoru

Harashima, Nanae

Harasym, Joanna

Harding, Ian

Hardingham, Jennifer E

Hargreaves, Justin Stephen James

Harijan, Rajesh K

Hariri-Ardebili, Mohammad Amin

Harmanci, Arif Ozgun

Haro, Diego

Harp, Joel M.

Harper, James

Harrison, Jonathan S.

Harrison, Paul H. M.

Harrison, Robert

Harvey-Samuel, Tim

Hasanuzzaman, Mirza

Hasegawa, Morifumi

Hashidzume, Akihito

Hashimoto, Makoto

Hashimoto, Masaru

Hassan, Mohamed

Hassan, Sherif T. S.

Hatae, Noriyuki

Hatami, Asa

Hatano, Tsutomu

Hatfield, Ronald D.

Hatterman-Valenti, Harlene

Hatzidimitriou, Efimia

Haudecoeur, Romain

Haugen, Havard Jostein

Hausmann, Michael

Hausner, Georg

Hawryl, Anna M.

Hay De Bettignies, Anne-Emmanuelle

Hay, Michael

Hayakawa, Takehito

Hayashi, Fumitaka

Hayashi, Shotaro

Hayashi, Yoshihito

Hayes, K C.

Hayn, Roland

Hazai, László

Hazak, Ora

He, Hailun 
He, Hongliang

$\mathrm{He}$, Jie

Heaney, Liam

Hearn, Michael J.

Heath, Timothy

Heber, Dieter

Hegedúsová, Alžbeta

Heider, Dominik

Heinbockel, Thomas

Heiner, Detert

Heipieper, Hermann

Heise, H.M.

Heiskanen, Juha

Helaly, Soleiman

Held, Christoph

Heleno, Sandrina

Hellwig, Michael

Helm, Richard

Heltzel, Jacob

Hemraz, Usha D.

Henderson, William

Hendriks, Wiljan

Henen, Morkos A.

Henkel, Janin

Henrique, Rui

Henry, Ryan A.

Hensel, Andreas

Hepnarova, Vendula

Her, Song

Herbert, John

Herczegh, Pál

Heredia-Guerrero, José Alejandro

Herker, Eva

Herman, Andrzej Przemysław

Hernández, Francisca

Hernández, Ignacio

Hernández-Córdoba, Manuel

Hernández-Hernández, Ángel

Hernández-Mesa, Maykel

Hernando, Jordi

Herndon, James W.

Herold, Nikolas

Herráez-Hernández, Rosa

Herrera, Raquel P.

Herrera-May, Agustín

Herrero, Baudilio

Herres-Pawlis, Sonja

Herrmann, Fabian

Herrmann, Mathias

Herwadkar, Anushree

Hester, Joanna

Hideg, Éva
Hiejima, Yusuke

Hikawa, Hidemasa

Hildén, Kristiina

Hildenbrand, Georg

Hildreth, Blake

Hildreth, Sherry

Hill, Daniel

Hill, J. Grant

Hillwig, Matthew

Hiltunen, Minna

Hinrichs, Wouter L.J.

Hirakawa, Hidehiko

Hirasaka, Katsuya

Hirasawa, Noriyasu

Hirayama, Fumitoshi

Hiroaki, Hidekazu

Hirota, Kiichi

Hirota, Yuichiro

Hixson, Josh

Ho, Eric S.

Ho, Junming

Ho, Mei-Lin

Ho, Mengfei

Ho, Shin-Lon

Ho, Su-Chen

Ho, Wing Shing

Ho, Yi-Cheng

Hoang, Nam V

Hocek, Michal

Hockey, Meghan

Hodge, Allison

Hoffman, Angela

Hoffmann, Klaus

Hofmann, Johann

Hofmann, Ute

Hoggard, Patrick E.

Hohensinner, Philipp

Hohmann, Judit

Holalu, Srinidhi

Holder, Alvin A.

Hollenhorst, Peter C

Holmes, Dawn E.

Holst, Otto

Holynska, Malgorzata

Holzmann, Klaus

Holzmann, Nicole

Homaeigohar, Shahin

Homma, Miwako Kato

Honda, Masaki

Hong, Jin-Long

Hong, Mee Young

Hong, Yanjun 
Hong, Yonggeun

Hong, Yuning

Honzíček, Jan

Höög, Johanna

Hoogeveen, Ellen K.

Hook, Ingrid

Hooper, Cornelia

Horax, Ronny

Horino, Yoshikazu

Horiuchi, Yasue

Horowitz, Rami A.

Horvai, George

Horvath, Dragos

Hoshino, Masaru

Hosomi, Ryota

Hosoya, Takamitsu

Hosoya, Takashi

Hossan, Mohammad Robiul

Hosseini, Abolfazl

Hoštálková, Anna

Hostaš, Jiř́

Hotta, Jun-ichi

Hou, Duen-Ren

Hou, Harvey

Hou, Jingwei

Hougland, James

Houng, Jer-Yiing

Hour, Mann-Jen

Houzé, Pascal

Howarth, Ashlee

Hoyos, Pilar

Hrabal, Richard

Hrnčič, Maša Knez

Hroboňová, Katarína

Hsia, Shih-Min

Hsiao, George

Hsieh, Changwei

Hsieh, Feng-Chia

Hsieh, Hsi-Lung

Hsieh, Pei-Wen

Hsin, Kun-Yi

$\mathrm{Hsu}, \mathrm{Fu}-\mathrm{Yin}$

Hsu, I-Jui

Hsu, Kuo-Sheng

Hsu, Shu-hao

Hsu, Sung-Po

Hsueh, Yi-Huang

$\mathrm{Hu}, \mathrm{Bo}$

$\mathrm{Hu}$, Guang

$\mathrm{Hu}$, Ping

$\mathrm{Hu}$, Qiwen

$\mathrm{Hu}$, Shang-Hsiu
$\mathrm{Hu}$, Wei-Ping

$\mathrm{Hu}$, Xiao

$\mathrm{Hu}$, Yong

$\mathrm{Hu}$, Zhenbin

Huang, Chi-Chang

Huang, Chih-Ching

Huang, Chih-Feng

Huang, Chih-Ling

Huang, Chiung-Cheng

Huang, Genin Gary

Huang, Guan-Jhong

Huang, Hao

Huang, Hsu-Shan

Huang, Hui-Chi

Huang, Hung-Chung

Huang, Jason C.

Huang, Jiann-Jyh

Huang, Jingfeng

Huang, Jui-Hsien

Huang, Lei

Huang, Ling

Huang, Shu-Chi

Huang, Wei-Jan

Huang, Wen-Chung

Huang, Wen-Lii

Huang, Wenlin

Huang, Xiao

Huang, Xueshi

Huang, Yucheng

Huang, Yufeng

Hubková, Beáta

Hübner, Florian

Huck, Christian

Huczyński, Adam

Hudson, Robert

Huefner, Antje

Hueso, Jose L.

Hughes, Richard K.

Huh, Do Sung

Huh, Jae-Won

Huh, Yun Suk

Humpl, Tilman

Hung, Che-Lun

Hung, Kuo-Hsiang

Hungerford, James

Huot, Philippe

Huppi, Konrad

Hur, Sun-Jin

Hurley, Margaret

Hussein, Waleed

Husson, Jérôme

Huszcza, Ewa 
Hutter, Michael

Hwang, Bang Yeon

Hwang, Long-Chih

Hwu, Jih-Ru

Hye, Jin Jung

Iacopetta, Domenico

Iacovino, Rosa

Iadarola, Paolo

Iannazzo, Daniela

Ianora, Adrianna

Iatsunskyi, Igor

Ibeas, José

Ibrahim, Salam A

Ichimura, Kazuya

Ientile, Riccardo

Iglesias, Luis E.

Iguchi, Taisen

Ihle, Andreas

Iijima, Noriaki

Ijima, Hiroyuki

Ikegami, Takahisa

Ikehara, Kenji

Ilagan, Ma. Xenia G.

Ilarraza, Ramses

Ileana Ionescu, Mihaela

Ilia, Gheorghe

Ilie, Ioana M.

Iliev, Boyan

Ilisz, István

Illa, Ona

Imaz, Inhar

Imhof, Petra

Imig, John

Imm, Jee-Young

Imokawa, Genji

Imperatore, Concetta

Imperlini, Esther

Inaba, Hiroshi

Inagaki, Hidetoshi

Inagaki, Naoki

Inagaki, Noritoshi

Inaguma, Shingo

Inamura, Kentaro

Indiveri, Cesare

Infante, Paola

Infantes Molina, Antonia

Ingram, Conrad

Ingrosso, Francesca

Inguimbert, Nicolas

Inngjerdingen, Marit

Inokuchi, Tsutomu

Inoue, Atsuko
Insenser, María

Inuzuka, Toshiyasu

Ioelovich, Michael Ya

Ionita, Petre

Ionut, Ioana A.

Iorizzi, Maria

Iossifidou, Eleni

Iqbal, Hafiz M.N.

Irakli, Maria

Iram, Surtaj

Iriepa Canalda, Isabel

Isaza Martínez, José Hipólito

Isemura, Mamoru

Ishigaki, Mika

Ishii, Takahiro

Iskierko, Zofia Ewa

Islam, M. Nurul

Islam, Md Soriful

Ismael, M. Isabel

Ismaili, Lhassane

Isman, Murray B.

Isovitsch, Ralph

Ita, Kevin

Ito, Hideyuki

Ito, Jun-ichi

Ito, Shingo

Ito, Takeru

Ito, Takuya

Ito, Yoichiro

Itoh, Takafumi

Itoh, Toshiyuki

Itou, Junji

Itsuno, Shinichi

Iturriaga-Vasquez, Patricio

Ivancevic, Atma

Ivanov, Alexander V

Ivanov, Andrey

Ivanov, Sergey M.

Ivanova, Bojidarka

Ivanova, Yordanka

Iwai, Atsushi

Iwamoto, Satoshi

Iwamoto, Shotaro

Iwanicki, Adam

Iwasa, Takeshi

Iwasaki, Takashi

Iwatsuki, Masato

Izadyar, Anahita

Izgu, Enver Cagri

Izquierdo-Rico, Maria José

Izsvák, Zsuzsanna

Izumi, Yudai 
Jabłońska-Trypuć, Agata

Jablonski, Miroslaw

Jackson, Michael A.

Jackson, William F.

Jacob, S. John

Jacobsen, Elisabeth Egholm

Jacquemin, Denis

Jadeja, Ravirajsinh Navalsinh

Jadhav, Gopal P.

Jaenicke, Stephan

Jaffrezic-Renault, Nicole

Jafra, Sylwia

Jagerovic, Nadine

Jagiello, Karolina

Jahandideh, Samad

Jahidul, Islam

Jain, Akshay

Jaiswal, Amit K.

Jaiswal, Jagdish

Jaiswal, Rakesh

Jakkle, Frieder

Jakóbik-Kolon, Agata

Jakopic, Jerneja

Jakubczyk, Anna

Jakubikova, Jana

Jalkanen, Aaro J.

James, Paul

Jamieson, Andrew

Jamieson, Stephen M. F.

Jampaiah, Deshetti

Jamroz, Michal

Jana, Navendu

Janakiraman, Harinarayanan

Janczarek, Monica

Jandrić, Zora

Jang, Byoung Kuk

Jang, Dae Sik

Janissen, Richard

Jankauskaite, Virginija

Janovák, László

Janowski, Miroslaw

Jansen, Rolf

Jantas, Danuta

Jaradat, Nidal

Jarmoluk, Andrzej

Jarosz-Wilkolazka, Anna

Jarret, Robert L.

Jarrous, Nayef

Jasicka-Misiak, Izabela

Jasik, Patryk

Jasinski, Marcin

Jasiński, Radomir
Jaumot, Joaquim

Javier, Sánchez-Nieves

Jayakumar, Rangasamy

Jayakumar, Thanasekaran

Jayamohan, Harikrishnan

Jayant, Rahul Dev

Je, Jae-Young

Jean, Ludovic

Jeandet, Philippe

Jeandroz, Sylvain

Jedynak, Katarzyna

Jee, JunGoo

Jelen, Henryk

Jelliss, Paul

Jena, Prasant Kumar

Jenett-Siems, Kristina

Jenkins, Ian

Jenkins, Samir V.

Jennings, Jessica Amber

Jensen, Lars Henrik

Jenssen, Håvard

Jeon, Byeong Hwa

Jeon, Hyeonyeol

Jeon, Noo Li

Jeon, Sung Ho

Jeong, Daewon

Jeong, Heon-Ho

Jeong, Ki Jun

Jeong, Lak Shin

Jeong, Seon-Yong

Jeong, Soo Hee

Jeremy, Teoh Yuen Chun

Jerković, Igor

Jerome, De Ruyck

Jeschke, Gunnar

Jetten, Anton

Jha, Mithilesh

Ji, Tianjiao

Jiang, Dianlu

Jiang, Jiang

Jiang, Junke

Jiang, Xuntian

Jiang, Yuan-Ye

Jiang, Zaixing

Jiao, Yinping

Jimbo, Mitsuru

Jimenez, M. Angeles

Jimenez, Randi

Jiménez-Alemán, Guillermo

Jiménez-Araujo, Ana

Jiménez-Aspee, Felipe

Jimenez-Barbero, Jesus 
Jimenez-lopez, Jose C.

Jin, Kailong

Jin, Zhao

Jindrich, Jindrich

Jirovetz, Leopold

Jo, Young Suk

João Ramalhosa, Maria

João, Benevides

John, Łukasz

Johnson, David

Jokić, Stela

Jolly, Pawan

Jones, Chris

Jones, David

Jones, Kathryn Marie

Jones, Marjorie

Jones, Meriel

Jonsson, Sofi

Joo, Hong-Gu

Jorda, Radek

Jordão, António Manuel

Jordao, Luisa

Jørgensen, Kåre

Jose, Jiney

Joshi, Hiren

Joshi, Pushpa Raj

Joshi, Sanket

Joubert, Fanny

Joule, John A.

Jovanović, Dragana

Jovanovic-Santa, Suzana

Joven, Jorge

Jówko, Ewa

Ju, Lu-Kwang

Juang, Shin-Hun

Judith, María Judith

Judson, Richard S

Jugessur, Aju

Juhasz, Bela

Julianna, Oláh

Juliano, Claudia

Jullian, Nathalie

Jullien, Frédéric

Jung, Chol-Hee

Jung, Jaehan

Jung, Seunho

Junior, Ary Fernandes

Juodkazis, Saulius

Jurado, Amalia S.

Jurado-Sánchez, Beatriz

Jurić, Marijana

Jurikova, Tunde
Jurman, Giuseppe

Juskowiak, Bernard

Justicia, José

Juszczak, Lesław

Juvik, John

K A Foyez, Mahmud

K. Hashmi, Stephen

Kabała-Dzik, Agata

Kabelác, Martin

Kabil, Omer

Kaczor, Agnieszka A.

Kaczorek, Ewa

Kadela-Tomanek, Monika

Kadokawa, Jun-ichi

Kadota, Isao

Kafarski, Pawel

Kafle, Lekhnath

Kahlert, Heike

Kahr, Bart

Kaiser, Leonard

Kaiser, Marcel

Kaja, Simon

Kajimoto, Shinji

Kajiya, Hiroshi

Kakkar, Ashok

Kalai, Tamas

Kalani, Komal

Kalayda, Ganna

Kalemba, Danuta

Kalhapure, Rahul S.

Kali, Gergely

Kaliniewicz, Zdzisław

Kalinin, Vladimir

Kalinowska Lis, Urszula

Kalinowska, Monika

Kallifidas, Dimitris

Kalogiannis, Stavros

Kalogirou, Andreas S.

Kalsi, Megha

Kaltner, Herbert

Kamagata, Kiyoto

Kambhampati, Siva Pramodh

Kamdem, Donatien Pascal

Kamdem, Jean Paul

Kamel, Karol

Kameo, Hajime

Kameyama, Atsushi

Kamiloglu, Senem

Kamitori, Shigehiro

Kamzolova, Svetlana

Kan, Chi-wai

Kanamori, Takao 
Kananovich, Dzmitry G.

Kanaujia, Parijat

Kanazawa, Arihiro

Kanbara, Takaki

Kanekiyo, Takahisa

Kanetis, Loukas

Kang, Chang Ho

Kang, CongBao

Kang, Hee Y.

Kang, Hui-Seung

Kang, Hyun Wook

Kang, Jae Seung

Kang, Jeon Woong

Kang, Jonghoon

Kang, Jun Yong

Kang, Kyungsu

Kang, Sinyoung

Kangas, Michael

Kano, Naoki

Kantminiene, Kristina

Kanzaki, Hiroyuki

Kao, Chai-Lin

Kao, Erl-Shyh

Kaparaju, Prasad

Kapusta-Duch, Joanna

Kar, Adwitiya

Karabagias, Ioannis

Karafiloglou, Padeleimon

Karakasidis, T.E.

Karamać, Magdalena

Karamanoli, Katerina

Kardos, József

Karim, Mohammad Rezaul

Karlovsky, Petr

Kårlund, Anna

Karmakar, Partha

Karnaouri, Anthi

Karousou, Evgenia

Karpiński, Tomasz M.

Kasem, Kasem K.

Kashina, Anna

Kashiwagi, Shinichiro

Kashman, Yoel

Kaškonienè, Vilma

Kasper, Dennis

Kasperkiewicz, Paulina

Kasten-Jolly, Jane

Kataev, Evgeny

Katahira, Rui

Kataoka, Hiroyuki

Kataoka, Naoyuki

Kataoka, Ryota
Katayama, Hironori

Katayama, Shigeru

Katepalli, Hari

Kathuria, Himanshu

Kato, Yasumasa

Katogi, Hideaki

Katsantonis, Dimitrios

Katsila, Theodora

Kattamuri, Padmanabha

Katuchova, Jana

Kaufman, Teodoro S.

Kauppinen, Anu

Kaveh, Hamed

Kavita, Kumari

Kawabata, Kyuichi

Kawabe, Akira

Kawada, Manabu

Kawaguchi, Shin-ichi

Kawaguchi, Toshikazu

Kawakami, Susumu

Kawakita, Hidetaka

Kawamura, Akira

Kawamura, Fumio

Kawamura, Izuru

Kawao, Naoyuki

Kawa-Rygielska, Joanna

Kawase, Yoshinori

Kawashima, Hideki

Kawashima, Yasushi

Kawato, Takayuki

Kawato, Yuji

Kay, Colin

Kay, Graeme

Kayano, Shin-ichi

Kazeto, Yukinori

Kazunori, Kadota

Kebede, Biniam T.

Kedage, Vivekananda

Keglevich, György

Keiler, Annekathrin Martina

Keith, Jason M.

Kéki, Sándor

Keller, Adrian

Keller, Andreas

Kello, Martin

Kellogg, Josh

Kelly Aparecida Dias, De Freitas Castro

Kelly, Christopher V.

Kemp, Michael G.

Keplinger, Tobias

Keppler, Julia

Kerch, Garry 
Kerimi, Asimina

Kerr, William

Kerrigan, Nessan J.

Kerrihard, Adrian L.

Kerwin, Sean M.

Kessel, David

Keszler, Agnes

Ketav Kulkarni, Ketav

Ketkar, Amit

Keum, Young-Soo

Kevadiya, Bhaveah

Keyzers, Rob

Khadka, Manoj

Khairullina, Veronika

Khalil, Ibrahim R.

Khalil, Zeinab

Khallouki, Farid

Khan, Mohammad Sharif

Khan, Mohsin

Khan, Mujeeb

Khan, Mushfiquddin

Khan, Naghma

Khan, Omar F.

Khan, Shafiq

Khatri, Bhuwan

Khatri, Kshitij

Khattab, Muhammad

Khaw, Kooi

Khisamutdinov, Emil

Khlebnikov, Andrei I.

Khodanovich, Marina

Khoddami, Ali

Khotimchenko, Maxim Y.

Khoury, Samar

Khurshid, Zohaib

Kiani, Amirkianoosh

Kicel, Agnieszka

Kiczorowska, Bożena

Kiec-Kononowicz, Katarzyna

Kiefer, Johannes

Kieffer, Bruno

Kieliszek, Marek

Kihara, Shin-ichi

Kijjoa, Anake

Kikowska, Małgorzata

Kikuchi, Haruhisa

Kikuchi, Macoto

Kilmartin, Paul

Kim, Bonglee

Kim, Chonsaeng

Kim, Choon Young

Kim, Chul Young
Kim, Chung Sub

Kim, Chyer

Kim, Daehwan

Kim, Dongin

Kim, Donguk

Kim, Hangun

Kim, Hoon

Kim, Hoy-Taek

Kim, Hyesun

Kim, Hyeung-Rak

Kim, Hyoungsu

Kim, Hyung Sik

Kim, Hyung-Joon

Kim, Hyunwoo

Kim, InKyeom

Kim, Jaheon

Kim, Jeongkwon

Kim, Ji Yeon

Kim, Joo-Hwan

Kim, Junheon

Kim, Keun-Sik

Kim, Ki Hyun

Kim, Kil-Soo

Kim, Kwanggi

Kim, Kyounghyun

Kim, Mi-hyun

Kim, Namshin

Kim, Ok Tae

Kim, Sang Hoon

Kim, Seok-Jhin

Kim, Soo Un

Kim, Sung-Koo

Kim, T. Doohun

Kim, Tae Kon

Kim, Tae-Hyung

Kim, Yang

Kim, Yongae

Kim, Youn-Chul

Kim, Yu Chul

Kim, Yunjeong

Kimira, Yosifumi

Kimura, Hideto

Kimura, Ken-ichi

Kimura, Seisuke

Kimura, Shunsaku

Kinaciyan, Tamar

Kinder, David

Kinoshita, Ayae

Kinoshita, Takayoshi

Kinthada, Ramakumar

Kintzel, Polly E.

Kinzhalov, Mikhail A. 
Kiparissides, Costas

Kipper, Karin

Kirienko, Natasha

Kirillov, Alexander M.

Kirillova, Alina

Kirsch, Gilbert H.

Kirton, Stewart B.

Kiryukhin, Maxim V.

Kiss, Anna

Kiss, János

Kiss, Nóra Zsuzsa

Kitagawa, Masatoshi

Kitagishi, Hiroaki

Kitahama, Yasutaka

Kitamura, Akira

Kitano, Yoshikazu

Kitazawa, Takafumi

Kiuru, Paula

Kizana, Eddy

Kjaergaard, Henrik

Klaerner, Frank-Gerrit

Klajnert-Maculewicz, Barbara

Klarskov, Klaus

Klavina, Laura

Kleier, Daniel A.

Klein, Axel

Klensporf-Pawlik, Dorota

Klose, Thomas

Klosterman, Jeremy

Kluza, Jérome

Klyubin, Igor

Kmetič, Ivana

Knapp, Spencer

Knez, Željko

Knight, Alan

Knight, David

Knippschild, Uwe

Knittelfelder, Oskar

Knölker, Hans-Joachim

Knoshaug, Eric

Knudsen, Gabriel Arther

Knupp, Gerd

Ko, Shun-Yao

Kobayakawa, Tatsu

Kobayashi, Kenichi

Kobayashi, Yayoi

Kobayashi, Yutaro

Kobori, Akio

Koch, Matthias

Koch, Wojciech

Kocot, Joanna

Kodama, Daisuke
Kodoudakis, Nikos

Koerner, Roman

Köfalvi, Attila

Kogan, Marcelo

Koh, Cho Yeow

Köhler, Karsten

Kohlmann, Holger

Kohyama, Noriko

Koizumi, Take-aki

Kojima, Hideo

Kojima-Yuasa, Akiko

Kok, Victor C.

Kokado, Kenta

Kokoška, Ladislav

Kokotos, Christoforos G.

Kokotos, George

Koksharova, Olga

Kolaczkowski, Bryan

Kolanowski, Jacek L.

Kolaric, Branko

Kolesárová, Adriana

Koller, Ulrich

Kolocouris, Antonios

Komaki, Hisayuki

Komasa, Satoshi

Komeyama, Kimihiro

Komilis, Dimitrios

Kommineni, Vally

Konar, Arkaprabha

Kong Thoo Lin, Paul

Kong, In-Soo

Kong, Lingxue

Kong, Qingke

Kong, Weili

Konieczna, Lucyna

König, Gerhard

Königs, Rene M.

Konno, Hiroyuki

Konno, Katsuhiro

Kontziampasis, Dimitrios

Koo, Byungjin

Kool, J

Kootala, Sujit

Kopec, Rachel

Kopecký, Vladimír

Kopel, Pavel

Kopjar, Mirela

Koppel, Kadri

Korb, Marcus

Korfei, Martina

Korkina, Ludmila G.

Kornprat, Peter 
Koroteev, Pavel S.

Korsching, Eberhard

Korstad, John

Korzeniowska, Margorzata

Košak, Aljoša

Koschella, Andreas

Koshino, Hiroyuki

Koslowski, Thorsten

Kostakis, George

Kostakis, Ioannis

Kostecka, Małgorzata

Kosterin, Oleg

Kosters, Astrid

Kostiainen, Mauri

Kosuge, Yasuhiro

Kotek, Jan

Kotiuga, Michele

Kotloski, Robert

Kotlowski, Roman

Kotsikorou, Evangelia

Kotsuchibashi, Yohei

Kotta-Loizou, Ioly

Kotula-Balak, Malgorzata

Koufaki, Maria

Koukounaras, Athanasios

Koulen, Peter

Koupenova, Milka

Koutelidakis, Antonios E.

Koutentis, Panayiotis A.

Koutselas, Ioannis

Kouwer, Paul

Kouza, Maksim

Kovac, Stjepana

Kovács, Lajos

Koval, Alexey

Kovalenko, Sergey

Kovinich, Nik

Kovvasu, Surya

Kowalak, Stanisław

Kowalczewski, Przemysław

Kowalska, Ewa

Kowalska, Katarzyna

Kowalska-Góralska, Monika

Kowalski, Konrad

Kowalski, Stefanjan

Koyama, Takeshi

Koyioni, Maria

Kozaki, Akiko

Kozakiewicz, Anna

Kozieł, Edmund

Koziel, Jacek A.

Kozlevčar, Bojan
Kozliak, Evguenii I.

Kozlov, Serguei

Kozłowska, Mariola

Kozuch, Sebastian

Kragović, Milan

Krajka-Kuźniak, Violetta

Kramer-Marek, Gabriela

Kranjac, Marina

Krasniewska, Karolina

Krchnak, Viktor

Kregiel, Dorota

Kremleva, Alena

Kren, Vladimir

Kressler, Joerg

Kries, Hajo

Krisch, Judit

Kristensen, Mie

Krivovichev, Vladimir

Krizbai, Istvan

Król, Ewelina

Król-Kilińska, Żaneta

Kroncke, Brett M

Krstin, Sonja

Kruger, Jacob S.

Kruszynski, Rafal

Krylov, Sergey N.

Kschischo, Maik

$\mathrm{Ku}$, Kang-Mo

$\mathrm{Ku}$, Seockmo

Kuan, Yu-Hsiang

Kuang, Huihui

Kubala, Lukáš

Kubatka, Peter

Kubica, Paweł

Kubíček, Vojtěch

Kubicova, Lenka

Kubina, Robert

Kubiński, Konrad

Kubo, Takanori

Kubota, Takaaki

Kuca, Kamil

Kucerova, Marta

Kucinska-Lipka, Justyna

Kuckling, Dirk

Kucuk, Israfil

Kucukkal, Tugba

Kuenz, Anja

Kuerten, Stefanie

Kues, Wilfried A.

Kugler, Szymon

Kuhlmann, Markus

Kuimova, Marina 
Kujawska, Małgorzata

Kujawski, Jacek

Kukli, Kaupo

Kukovec, Boris-Marko

Kukreja, Muskan

Kukshal, Vandna

Kukula-Koch, Wirginia

Kulkarni, Shashank

Kulma, Anna

Kumar, Amit

Kumar, Dhiraj

Kumar, Krishan

Kumar, Manu

Kumar, Narender

Kumar, Narendra

Kumar, Nitin

Kumar, Pradeep

Kumar, Prasun

Kumar, Sunil

Kumari, Asha

Kung, Chung-Wei

Kunjukunju, Sangeetha

Kuno, Toshiya

Kunz, Horst

Kunz, Wolfram

Kuo, Chung-Wen

Kuo, Meng-I

Kuo, Ping-Chung

Kuo, Shiao-Wei

Kuo, Yao-Haur

Kupczyński, Robert

Küppers, Stephan

Kurdowska, Anna K.

Kurek, Marcin

Kuroboshi, Manabu

Kuroda, Chiaki

Kurtán, Tibor

Kuś, Piotr

Kusch, Peter

Kushkevych, Ivan

Kuttel, Mary

Kuwahara, Masayasu

Kuyukina, Maria S.

Kuźnik, Nikodem

Kuzuhara, Daiki

Kuzuya, Akinori

Kwak, Jong Hwan

Kwak, Kyungwon

Kwak, Seonyeong

Kwakowsky, Andrea

Kwan, Ann

Kwasniewska, Jolanta
Kweon, Oh-Kyeong

Kwiatkowska, Katarzyna

Kwiatkowski, Miroslaw

Kwiecień, Iwona

Kwo, Hang Fai

Kwon, Soon-Hong

Kwon, Young-Wan

Kyle, Stuart

Kynický, Jindřich

Kyzioł, Karol

La Barbera, Giorgia

La Ferla, Barbara

La Motta, Concettina

La Penna, Giovanni

La Russa, Daniele

La Spada, Luigi

Laarmann, Tim

Labbadia, John

LaBean, Thomas $\mathrm{H}$.

Labrou, Nikolaos

Labruna, Giuseppe

Lachenmeier, Dirk

Lachman, Jaromir

Lachowicz, Joanna Izabela

Lachowicz, Sabina

Lafarga, Tomas

Lagana, Aldo

Lago, João Henrique Ghilardi

Lahooti, Hooshang

Lai, Qinghua

Lai, Yandong

Laird, Eamon

Laity, Peter R

Lakard, Boris

Lalas, Stavros

Lallana, Enrique

Lam, Kim-Hung

Lam, Kwok Ho

Lambert, Joshua

Lambert, Nevin A.

Lambrinidis, George

Lamzin, Victor

Lan, Lan

Lan, Wenjian

Lan, Yu

Lan, Yucheng

Lanças, Fernando

Lancelin, Jean-marc

Landi, Marco

Lang, Siegmund

Lange, Heiko

Langendorf, Christopher 
Langer, Jerzy J

Langer, Thierry

Langford, T. Dianne

Lannoy, Damien

Lanzerstorfer, Peter

Lappan, Uwe

Laranjinha, João Antonio

Lara-Sanchez, Agustin

Larobina, Domenico

Laronze-Cochard, Marie

Larraneta Landa, Eneko

Larson, Nicholas B.

Lash, Timothy

Łatka, Leszek

Latosińska, Jolanta Natalia

Latouche, Camille

Lattanzio, Rossano

Lau, Christopher S.

Laube, Markus

Launay, Jean Marie

Laurent, Ferrié

Laurenti, Enzo

Lauro, Maria Rosaria

Lauschke, Volker

Laustsen, Andreas Hougaard

Lavandera, José Luis

Lavelli, Vera

Lavilla, Rodolfo

Lavoie, Serge

Lázár, István

Lazarus, Kyren

Lazzarato, Loretta

Lazzari, Claudio

Lazzaroni, Roberto

Le Borgne, Marc

Le Bourvellec, Carine

Le Ouay, Benjamin

Le Questel, Jean-yves

Le Roes-Hill, Marilize

Le, Hoang-Thanh

Leadbeater, Nicholas

Leal, Ana Mendes

Lear, Benjamin J.

Lebar, Matthew

Lebeau, Luc

Lebedev, Nikolai

Leblebici, Mumin Enis

Lecaille, Fabien

Leclercq, Guy

Leclercq, Loïc

Lecomte, Philippe

Lederkremer, Gerardo Z.
Ledo, Ana

Ledwon, Przemyslaw

Lee, Bong Ho

Lee, Bor-Shiunn

Lee, Changwon

Lee, Che-Hsin

Lee, Cheng-I

Lee, Chen-Yu

Lee, Chia-Hung

Lee, Ching-Kuo

Lee, E-jian

Lee, Gihyun

Lee, Gyo

Lee, Hian Kee

Lee, Hong In

Lee, Huei-Jane

Lee, Hye Suk

Lee, Hyunbok

Lee, Ilkeun

Lee, Jae Wook

Lee, Jaehwi

Lee, Jae-Suk

Lee, Jin-Koo

Lee, Jin-Kyun

Lee, Jiun-haw

Lee, Jongsung

Lee, Joo Hyoung

Lee, Jun Seok

Lee, Jun Sik

Lee, Keun-Hyeung

Lee, Kwang Ho

Lee, Meng-Jen

Lee, Meng-shiou

Lee, Mi Kyeong

Lee, Min Won

Lee, Sang Gil

Lee, Sang-Han

Lee, Sanghyeob

Lee, Sangkil

Lee, Sang-Myung

Lee, Seok Jae

Lee, Shih-yu

Lee, Soo Young

Lee, Sung Ho

Lee, T. Randall

Lee, Tai-Lin

Lee, Yeon-Ju

Lee, Yong Rok

Lee, Zhenghong

Lefebvre, Frederic

Lefebvre, Tony

Lefkimmiatis, Konstantinos 
Legentil, Laurent

Léger, Bastien

Legrand, François Xavier

Lehmann, Helmar

Lehner, Małgorzata

Lehotay, Steven J.

Lei, Fan

Lein, Matthias

Leitão, Ana

Leitão, Jorge H.

Leite Sampaio, Bruno

Lekka, Malgorzata

Lemeune, Alla

Lemli, Beáta

Lemoine, Pierric

Lemon, Christopher

Lemos, Manuel L.

Len, Christophe

Lenardão, Eder J.

Lenart, Andrzej

Lentini, Giovanni

Lenzen, Sigurd

León, Josefa

Leon, Juan Francisco

Leonard, Stephen

Leong, Max K.

Leonidas, Demetres D.

Leonidas, Palilis

Leonzio, Grazia

Lepenies, Bernd

Leporatti, Stefano

Lepore, Maria

Leroy, Baptiste

Lesner, Adam

Lesniewska, Eric

Lessard, Benoit

Lesyk, Roman B.

Leung, Chung-Hang

Leung, Ivanhoe

Leung, Ka Ho

Leung, Yuk-man

Lewandowicz, Grażyna

Lewandowski, Mikołaj

Lewinska, Anna

Lewis, Karen

Lewis, Kathy

Lewis, Randolph

Lewkowicz, Elizabeth

Lewkowski, Jarosław A.

Ley, Alexandra C.

Leymarie, Olivier

Lézot, Frédéric
Li, Ailin

Li, Cheng

Li, Chunshun

Li, Degang

Li, Fu-shuang

Li, Guohui

Li, Hou-Jin

Li, Hua-Bin

$\mathrm{Li}$, Jie

$\mathrm{Li}$, Jin

Li, Jinbo

Li, Jinghao

Li, Kaichang

Li, Kuo-Bin

Li, Mei-Ling

Li, Miaomiao

Li, Mingqiang

$\mathrm{Li}, \mathrm{Na}$ (China)

Li, Na (Macao, China)

Li, Ningyang

Li, Ping

Li, Shao

Li, Shao-Gang

Li, Shujun

Li, Siheng

Li, Tianhu

Li, Wei

Li, Wen-Wu

Li, Wenyi

Li, Xiang-Guo

Li, Xiaohua

Li, Xiaopeng

Li, Xinghui

Li, Yan (China)

Li, Yan (New Zealand)

Li, Yan (Singapore)

Li, Yang

Li, Yanmei

Li, Yan-Mei

Li, Yifan

Li, Yin

Li, Ying

Li, Yiwen

Li, Zhi-Minhg

Li, Zhonghu

Li, Zijian

Liagre, Bertrand

Liang, Kang

Liang, Yuerong

Liang, Zhibin

Liao, Jyh-fei

Liao, Pei-Chun 
Liao, Shu-Chuan

Liao, Wupeng

Liao, Yi-Jen

Liberto, Erica

Licciardello, Grazia

Licea Navaro, Alexei Fedorovish

Liddelow, Shane

Lidon, Fernando

Likozar, Blaž

Lim, Beong Ou

Lim, Dong Chan

Lim, Ji-Hong

Lim, Junxian

Lim, Yoongho

Lima, Marcos Dos Santos

Lima-Neto, Benedito

Lin, Chia-Her

Lin, Chih-Chien

Lin, Chih-Li

Lin, Ching-Hsuan

Lin, Chiu-Yue

Lin, Chung-Ho

Lin, Erick

Lin, Guiting

Lin, Hai-Shu

Lin, Jung-Chun

Lin, Kuo-Shyan

Lin, Liang-Tzung

Lin, Ligen

Lin, Long-Liu

Lin, Mei-Hsiang

Lin, Mei-huey

Lin, Shih-Yi

Lin, Shwu-jiuan

Lin, Shyh-Hsiang

Lin, Tzu-En

Lin, Xiaojie

Lin, Yu-Shen

Lin, Zekai

Lin, Zhoumeng

Lin, Zongtao

Lincoln, Per

Lindemann, Peter

Lindequist, Ulrike

Lindner, Robert

Lindsey-Boltz, Laura

Linet, Wolfgang

Ling, Maurice

Linscheid, Michael W.

Lionetti, Lillà

Liou, Ying-ming

Lisciani, Silvia
Litinas, Konstantinos

Litvić, Mladen

Liu, Baiquan

Liu, Cheuk Lun

Liu, Cong

Liu, Delong

Liu, Dong-hong

Liu, Gang

Liu, Guanglei

Liu, Guang-Yaw

Liu, Guoshi

Liu, Hai-yang

Liu, Hong

Liu, Hui-kang

Liu, Huolong

Liu, Jiemin

Liu, Juewen

Liu, Jun

Liu, Jun-Jen

Liu, Lei

Liu, Qiang

Liu, Qing-Song

Liu, Shing-Hwa

Liu, Shiuh-Tzung

Liu, Wenshe

Liu, Xiaoxi (USA)

Liu, Xiaoxi (USA)

Liu, Xifeng

Liu, Xuewei

Liu, Yanbiao

Liu, Yang

Liu, Yongqiang

Liu, Yongqing

Liu, Yu

Liu, Yuan-Shuai

Liu, Yujun

Liu, Yunling

Liu, Zhichao

Liu, Zhiqiu

Liu, Zhixia

Livaniou, Evangelia

Livesey, Geoffrey

Lizardi-Mendoza, Jaime

Llop, Jordi

Llorens, Eugenio

Lloyd, Matthew

Lo Meo, Paolo

Lo Scalzo, Roberto

Lo, Kai-Yin

Loboda, Agnieszka

Locatelli, Clinio

Locatelli, Marcello 
Lodyga-Chruscinska, Elżbieta

Lognay, Georges

Loh, Xian Jun

Lohezic-Le Devehat, Françoise

Lohman, Jeremy

Lohning, Anna

Lojou, Elisabeth

Lombó, Felipe

London, Edra

Long, Eric C.

Longo, Rocco

Longstaffe, James G.

Lönnberg, Harri

Lopes, Pedro E M

López Fernández, Hugo

Lopez Rodilla, Jesus M.

Lopez, Cristobal

López, María L.

Lopez-Escamez, Jose A.

López-García, Jorge

López-Santos, Carmen

Lopez-Viñas, Eduardo

Loppi, Steffano

Lorberboum-Galski, Haya

Lorenz, Peter

Lorenzo Abad, María Encarnación

Loretz, Brigitta

Losada-Perez, Patricia

Lotina-Hennsen, Blas

Lotti, Marina

Lou, HongXiang

Lou, Song

Louarn, Essyllt

Loureiro, Jose Miguel

Lourenço, Ana

Louro, Ricardo

Lovell, Jonathan

Loveridge, E. Joel

Loveridge, Joel

Lovrecic, Mercedes

Lowary, Todd

Lozano Ojalvo, Daniel

Lu, Chun-Ping

Lu, Hsu-Feng

$\mathrm{Lu}$, Huasong

$\mathrm{Lu}$, Jun

Lu, Mei-Chin

Lu, Qi

$\mathrm{Lu}$, Shenzhou

$\mathrm{Lu}$, Weiyue

$\mathrm{Lu}, \mathrm{Yi}$

Lu, Zhanping
Lu, Zhongbing

Lubal, Přemysl

Luber, Sandra

Lubin-Germain, Nadège

Luca, Oana

Lucaciu, Constantin Mihai

Lucarini, Massimo

Lucas, Kurt

Lucas, Xavier

Lucassen, Paul

Lucena, Rafael

Luchinat, Enrico

Lucia Tornesello, Anna

Lucini, Luigi

Luck, Rudy

Łuczak, Justyna

Ludwiczuk, Agnieszka

Lugo-Cervantes, Eugenia

Lui, Edmund Man King

Luís, Ângelo

Luis, Valencia Cabrera

Łukajtis, Rafał

Łukasik, Rafał

Lukaszkowicz, Krzysztof

Lukinavicius, Grazvydas

Lukyanov, Pavel

Luliński, Sergiusz

Lung, Ming-Yeou

Luo, Lyna

Luo, Minkui

Luo, Rongcong

Luo, Zisheng

Luo, Zonghua

Luque, F. Javier

Luque, Rafael

Luscombe, Christine

Luthra, Amit

Lütticken, Rudolf

Lutz, Martin

Luukkonen, Tero

Luxen, André

Lykakis, Ioannis

Lykidis, Charalampos

Lyu, Hui

Lyutakov, Oleksiy

M. Keseru, Gyorgy

$\mathrm{Ma}, \mathrm{Chao}$

Ma, Cong

Ma, Guojia

Ma, Liang

Ma, Qin

Ma, Tianyi 
Ma, Yong

Ma, Zexu

Ma, Zhikun

Maak, Steffen

Maász, Gábor

Mabou Tagne, Alex

Maccallini, Cristina

Macchi, Beatrice

Macchiarulo, Antonio

Maccione, Elias

Macernis, Mindaugas

Machetti, Fabrizio

Macia, Angela

Mackereth, Cameron

Maçôas, Ermelinda

Macone, Alberto

Macwan, Isaac

Mączka, Wanda

Madan, Vanesa

Maddau, Lucia

Madesis, Panagiotis

Madine, Jillian

Madrid, Alejandro

Madsen, Andreas S.

Mady, Mohamed F.

Madzhidov, Timur

Maeda, Tomoki

Maeng, Han-Joo

Mafra, Marcos Rogério

Mafu, Sibongile

Magain, Nicolas

Magalhães, Júlia M.C.S.

Magana, Luis Fernando

Magata, Yasuhiro

MaGee, David

Maggioni, Daniela

Magiorkinis, Emmanouil

Magnabosco, Giulia

Magnaldo, Thierry

Magni, Paolo

Magnus, Marcin

Magri, David C.

Magriotis, Plato A.

Mahal, Katharina

Mahavadi, Poornima

Maher, Pamela

Mahimainathan, Lenin

Mahmoud, Alaa El Din

Mahmudov, Kamran T.

Mahrwald, Rainer

Maican, Edmond

Maicas, Sergi
Mainero Rocca, Lucia

Maisch, Tim

Majewski, Leszek

Majireck, Max

Majkowska-Skrobek, Grażyna

Majtan, Juraj

Majumder, Kaustav

Makarska-Bialokoz, Magdalena

Makena, Monish

Maker, Garth

Malaguarnera, Giulia

Malawska, Barbara

Malde, Alpesh

Malekigorji, Maryam

Maleknia, Simin D

Malla, Spundana

Mallavia, Ricardo

Mallipeddi, Prema Latha

Malmberg, Per

Malmström, Lars

Maloberti, Alessandro

Malterud, Karl

Mamane, Victor

Manabe, Kei

Manavalan, Balachandran

Manca, Maria Letizia

Mancera, Ricardo L.

Mancianti, Francesca

Mancini, Ines

Mancini, Rock

Mancini, Simone

Manciu, Felicia

Manconi, Maria

Mancuso, Raffaella

Mandadi, Kranthi

Mandal, Debashis

Mandal, Soumyajit

Mandalari, Giusy

Mandic, Zoran

Mandity, Istvan

Mandoli, Alessandro

Mandrone, Manuela

Manfredini, Stefano

Mangalagiu, Ionel

Manian, Avinash P.

Manjunath, Manubolu

Mankin, Richard W.

Manley, Suliana

Mann, Francis

Manner, Suvi

Mano, Yuji

Manoury, Eric 
Manríquez-Torres, José De Jesús

Manthey, John A.

Mantle, Peter

Mantzioris, Evangeline

Mantzouridou, Fani

Manzano, Blanca R.

Manzhos, Sergei

Manzone, Marco

Mao, Meng (Australia)

Mao, Meng (USA)

Mao, Zongwan

Marangon, Matteo

Marasco, Daniela

Marasini, Nirmal

Marazzi, Marco

Marc, Daniel

Marceau, François

Marcelis, Lionel

Marchal, Richard

Marchesan, Silvia

Marcin Henryk, Struszczyk

Marco, Francisco

Marco-Contelles, José

Marek, Antonin

Marestin, Catherine

Margherita, Maioli

Mari, Michele

Maria, Coros

Maria, Diaconeasa Zoriţa

Marichev, Kostiantyn

Marin, Luminita

Marín, Mercedes

Marincs, Ferenc

Marini, Simone

Marino, Tiziana

Marjanović, Marko

Márki, Árpád

Markiewicz, Wojciech T.

Markkanen, Enni

Markopoulou, Olga

Marla, Sandeep

Marqués Villavecchia, Ana M.

Marra, Alberto

Marrelli, Mariangela

Marrocchi, Assunta

Marrone, Alessandro

Marschner, Christoph

Marszalek, Krystian

Marta, Ferreiro-González

Martelli, Pier Luigi

Martí, Sergio

Martín, Antonio E.
Martin, Finian

Martin, Jessica

Martin, Jose Miguel

Martin, Laetitia

Martin, Nathaniel

Martín-Antonio, Beatriz

Martín-Aragón, Sagrario

Martinelli, Adriano

Martinello, Marianna

Martínez Vázquez, Rafael Fernando

Martínez, Alberto

Martinez, Ana

Martínez, Ana

Martínez, Antonio

Martinez, Fleming

Martínez, Sidonia

Martínez-Ávila, Guillermo

Martinez-del-Pozo, Alvaro

Martinez-Gil, Luis

Martínez-Gómez, Pedro

Martinez-Lostao, Luis

Martin-Fontecha, Alfonso

Martín-Hernández, David

Martini, Claudia

Martini, Daniela

Martínková, Ludmila

Martino, Marco

Martín-Peláez, Sandra

Martín-Ramos, Pablo

Martins, Alice M.

Martins, Ian James

Martins, Luísa Margarida

Martins, Maria Rosario

Martins, Natália

Martins, Sonia

Martins-Lopes, Paula

Marto, Joana

Martoïa, Florian

Martorana, Alessandro

Maru, Marcia M.

Maruf, Abdullah Al

Maruyama, Ichiro

Maruyama, Kei

Marverti, Gaetano

Maryasin, Boris

Marycz, Krzysztof

Marzaro, Giovanni

Marzo, Tiziano

Marzocco, Stefania

Masek, Anna

Mashima, Ryuichi

Masi, Marco 
Masino, Francesca

Maslov, Mikhail A.

Masłyk, Maciej

Massa, Antonio

Massimi, Isabella

Massiot, Georges

Másson, Már

Massoud, Salah

Mastanjević, Kristina

Mastinu, Andrea

Mastrangelo, Anna M.

Masu, Hyuma

Masuda, Kiyoshi

Masui, Kenta

Masullo, Mariorosario

Mata, Ernesto

Mata, Jose A.

Mata, Rachel

Mata, Ricardo

Matczak-Jon, Ewa

Mateo, Cesar

Mateos, Raquel

Materska, Małgorzata

Mathé, Ewy A.

Matiadis, Dimitris

Matilla, Miguel

Matilla-Hernández, Antonio

Matos Lale, Juan

Matosiuk, Dariusz

Matouskova, Petra

Matsubara, Kouki

Matsuda, Fumio

Matsufuji, Hiroshi

Matsugo, Seiichi

Matsui, Daisuke

Matsumoto, Arimasa

Matsumoto, Ken'ichiro

Matsumoto, Kozo

Matsumoto, Shoji

Matsumoto, Yasuhiko

Matsuo, Takashi

Matsuo, Yosuke

Matsuo, Yukiko

Matsuura, Bunzo

Matsuura, Kazunori

Matta, Cherif

Mattevi, Andrea

Melzig, Matthias F.

Mattoo, Autar

Matts, Robert

Matwijczuk, Arkadiusz

Mauricio, Cuellar
Mavromoustakos, Thomas

Mayhan, William G

Mazerska, Zofia

Mazur-Bialy, Agnieszka Irena

Mazurek, Sylwester

Mazzoni, Luca

Mazzoni, Rita

Mazzotti, Fabio

McCarthy, Elizabeth

McCarthy, Pumtiwitt

McDaniel, Jonathan

Mcdougal, Owen M.

McDougall, Carmel

McFeeters, Robert

McGlinchey, Michael J.

McGonigal, Paul R.

McGowan, Eileen

McGowan, Sheena

McKeague, Maureen

McKee, Lauren S.

McLaughlin, Mark

McMorran, David

McMullin, David R.

Polidori, M. Cristina

Meadus, William Jon

Meana-Pañeda, Rubén

Medarde, Manuel

Medeiros, Adriane Bianchi Pedroni

Medellin-Castillo, Nahum

Medici, Serenella

Medina, Milagros

Medina-Vera, Myriam

Medrano, Hipolito

Medvidović-Kosanović, Martina

Meegan, Mary

Meenashisundaram, Ganesh Kumar

Megighian, Aram

Megyeri, Klara

Mehaffy, Carolina

Mehbub, Mohammad Ferdous

Mehdi, Ahmad

Mehta, Akul Y.

Meier-Menches, Samuel M.

Meijsing, Sebastiaan

Meisrimler, Claudia-Nicole

Mekinić, Ivana Generalić

Mele, Andrea

Melguizo-Guijarro, Manuel

Melini, Valentina

Mella Raipan, Jaime

Mellbye, Brett L.

Melo, André 
MENA, Pedro

Menabue, Ledi

Mencherini, Teresa

Mendes, Adriano

Mendes, Livia P.

Méndez, Lucía

Mendez-Sanchez, Nahun

Mendiola, José

Mendive-Tapia, David

Mendonca, Antonio

Mendoza-Nuñez, Victor Manuel

Meneghetti, Fiorella

Menet, Marie Claude

Menezes, Irwin Rose Alencar

Menezes, José

Menezes, Regina

Menna, Marialuisa

Menon, Jyothi

Mercer, Jason

Merfort, Irmgard

Mergler, Stefan

Merino, Gracia

Mernyák, Erzsébet

Merten, Christian

Mertens, Fredrik

Meschwitz, Susan

Mesgar, Milad

Messali, Mouslim

Messyasz, Beata

Mester, Patrick-Julian

Metzger, Robert M.

Metzinger, Laurent

Meyerholz, David K.

Mézes, Miklós

Mező, Gábor

$\mathrm{Mi}$, Lan

Mi, Xiaocheng

Miao, Tianxin

Michalska, Anna

Michalski, Rajmund

Michaud, Philippe

Micheau, Olivier

Michel, Martin C.

Michel, Piotr

Micheletti, Gabriele

Michels, Alexander J.

Michiho, Ito

Micillo, Raffaella

Mickelson, Alan R.

Miękus, Natalia

Miele, Adriana E.

Mielenz, Manfred
Miguel, Maria

Miki, Koji

Miki, Yasuhiro

Mikołajczyk-Bator, Katarzyna

Mikstacka, Renata

Mikulic-Petkovsek, Maja

Mikulskis, Paulius

Mikuš, Peter

Milano, Giuseppina

Milas, Haralampos N.

Milella, Luigi

Miles, Wayne Owen

Milia, Egle

Milledge, John J.

Miller, John $\mathrm{H}$.

Miller, Justin

Millet, Oscar

Millo, Enrico

Mimaki, Yoshihiro

Minami, Yasunori

Minamoto, Toshinari

Minato, Ken-ichiro

Minato, Makoto

Minceva, Mirjana

Mine, Yuichi

Minehan, Thomas G.

Minervini, Giovanni

Minetti, Giampaolo

Minjares-Fuentes, Jose Rafael

Minkiewicz, Piotr

Minnaar, Phillip

Minoru, Hayashi

Mintcheva, Neli

Minucci, Sergio

Minutolo, Filippo

Mirali, Mahla

Miranda, Cristobal

Miranda, Jose M.

Miranda, Katrina

Miras-Avalos, Jose Manuel

Mironescu, Monica

Mirossay, Ladislav

Mirzayans, Razmik

Mishin, Alexander S.

Mishra, Geetesh Kumar

Mishra, Nigam

Mishra, Rupesh K.

Mishra, Sandhya

Mishra, Sasmita

Misicka, Aleksandra

Misurcova, Ladislava

Mita, Damiano Gustavo 
Mita, Giovanni

Mitakou, Sofia

Mitchell, John

Mitev, Pavlin D.

Mitryukovskiy, Sergey

Mitsudo, Koichi

Mittal, Gayatri

Miura, Toshiko

Miyabe, Hideto

Miyagi, Atsuko

Miyamoto, Hirotaka

Miyamoto, Koji

Miyata, Yasuyoshi

Miyata, Yoshihiko

Miyazato, Hironari

Mizielińska, Małgorzata

Mizuno, Cassia S

Mizuno, Takayuki

Mizuta, Kentaro

Mladěnka, Přemysl

Mladenovic, Milan

Mlejnek, Petr

Mnif, Wissem

Mobley, Justin

Mocan, Andrei

Mochona, Bereket

Mockaitis, Gustavo

Modahl, Cassandra

Modec, Barbara

Modhiran, Naphak

Modugno, Francesca

Modukuri, Ramkumar

Moen, Erika

Mogielnicki, Andrzej

Mohamed, Sharmarke

Mohammadiarani, Hossein

Mohammed, Yousuf

Mohankumar, Kumaravel

Mohanram, Harini

Mohanta, Tapan

Moine, Laurence

Moineau-Chane-Ching, Kathleen I.

Mojsoska, Biljana

Mojzych, Mariusz

Mok, Daniel Kam-Wah

Moliner Martinez, Yolanda

Mollace, Vincenzo

Mollapour, Mehdi

Mollica, Adriano

Molnar, Maja

Molognoni, Luciano

Moloney, Mark G.
Molugu, Sudheer Kumar

Momken, Iman

Monaghan, Daniel

Monari, Antonio

Monchaud, David

Monclus, Michel

Mondejar, Maria E.

Monflier, Eric

Mong, Mei-Chin

Moni, Lisa

Monika, Waksmundzka-Hajnos

Moniuszko-Szajwaj, Barbara

Monroe, Mary Beth Browning

Montag, Judith

Montalbán, Mercedes G.

Montaño Priede, José Luis

Monteil-Rivera, Fanny

Monteleone, Stefania

Montenegro, Lucia

Montero, Olimpio

Montesano, Domenico

Montesarchio, Daniela

Montgomery, Magdalene

Montowska, Magdalena

Moody, David E

Moon, Byeong Cheol

Moon, Doo Kyung

Moon, Ilsoo

Moon, Kyung

Moore, John

Moore, Robert J.

Moore, Stuart

Morais, Mauricio

Morales, Gabriel

Morales-González, José Antonio

Morales-Morales, David

Morán, José M.

Morávková, Zuzana

Moreau, Regis

Moreira, José

Morelli, Sabrina

Moreno, Andrés

Moreno, Antonio D.

Moreno, Diego A.

Moreno, Miguel

Morganti, Pierfrancesco

Mori, Boyd

Mori, Mattia

Mori, Seiji

Mori, Shigeki

Morigi, Rita

Moriguchi, Takaya 
Morikawa, Toshio

Morimoto, Masanori

Morimoto, Yuma

Morimura, Shigeru

Morita, Naoki

Morkunas, Iwona

Moro, Loredana

Morrill, Louis C.

Morris, Gordon

Morris, James L.

Mortier, Jérémie

Morton, David

Morzycki, Jacek W.

Mosawy, Sapha

Moschou, Panagiotis N.

Moscobe, Danila

Mosquera, Marta

Mossine, Valeri V.

Most, Jasper

Mostafavi, Mehran

Mot, Augustin

Mota, Antonio J.

Mota, Manuel

Mouahid, Adil

Moura, Maria J.

Mourtas, Spyridon

Mourtzinos, Ioannis

Movassagh, Hesam

Moyano, Albert

Moyano, Lourdes

Mphahlele, Malose Jack

Mrówczyński, Radosław

Msaada, Kamel

Mucha, Artur

Mucsi, Zoltán

Muehlmann, Luis Alexandre

Mueller, Anja

Mueller, Wolf C.

Muggia, Lucia

Muhlack, Richard

Mukaida, Naofumi

Mukherjee, Soumya

Mulas, Maurizio

Mulet, J. M.

MULLER, Christian

Müller, Karsten

Muller, Patricia A. J.

Müller, Werner E.G.

Mulloy, Barbara

Mun, Jiyoung

Muñiz Rodriguez, Pilar

Munteanu, Bogdanel Silvestru
Muramoto, Koji

Murata, Jun

Murata, Toshihiro

Muresan, Vlad

Murillo Tovar, Mario Alfonso

Murkovic, Michael

Murotomi, Kazutoshi

Murphree, S. Shaun

Murphy, Cormac

Murphy, Neil

Murray, Jane

Murugaiyan, Jayaseelan

Murugan, N. Arul

Muruganandan, Shanmugam

Muschiol, Jan

Musgrave, Ian

Musiol, Robert

Musmarra, Dino

Musso, Ioana

Muszyńska, Bożena

Muthukumar, Thangavelu

Muthuramu, Ilayaraja

Mutin, Hubert

Muziol, Tadeusz Mikolaj

Mwinyi, Jessica

Myasoedova, Veronika

Myers, Stephen

Myllykallio, Hannu

Myrianthopoulos, Vassilios

Myśliwiec, Hanna

Mystkowska, Joanna

Mystrioti, C.

N. Setzer, William

$\mathrm{Na}$, Dong Hee

Nabissi, Massimo

Nabok, Alexei

Naccarato, Attilio

Naesens, Lieve

Nagai, Kouhei

Nagai, Yasuki

Nagai, Yoshinori

Nagaraj, Chandran

Nagasawa, Kazuo

Nagatsugi, Fumi

Nagel, Raimund

Nagy, Istvan

Naha, Pratap C.

Nahid, Abdullah-Al

Nair, Remya

Nair, Vimal

Nairz, Manfred

Najlah, Mohammad 
Nak Cheon, Jeong

Nakagawa-izumi, Akiko

Nakai, Yumi

Nakajima, Masahiro

Nakajima, Masatoshi

Nakamura, Itaru

Nakanishi, Waka

Nakano, Koji

Nakao, Yoichi

Nakashima, Souichi

Nakata, Norio

Nakata, Satoshi

Nakatani, Yoshihiko

Nakatsu, Kanji

Nallasamy, Palanisamy

Nam, Joo-Won

Nam, Ju-Ock

Nam, Kwangho

Nam, Sang-Ho

Nam, Sang-Jip

Namasivayam, Vigneshwaran

Namgaladze, Dmitry

Namiesnik, Jacek

Namieśnik, Jacek

Nanbu, Shinkoh

Nandha Premnath, Padmavathy

Nandi, Saikat (Sweden)

Nandi, Saikat (USA)

Nanev, Christo

Nanni, Loris

Nano, Adela

Narayan, Prakash

Narayanan, Ganesh

Nardi, Monica

Nardi, Serenella

Nardi, Tiziana

Nariai, Tadashi

Narita, Akihiro

Narsimhan, Ganesan

Narváez, Manuel

Nasef, Noha Ahmed

Nastasa, Cristina

Natadra Tabudravu, Jioji

Nath, Arijit

Natskoulis, Pantelis

Naughton, Declan

Naumann, Stefan

Naumova, Ella A.

Naumovski, Nenad

Navacchia, Maria Luisa

Navarrete-Vazquez, Gabriel

Navarro, Rodrigo
Naviglio, Daniele

Nawaz, Muhammad

Nawirska-Olszańska, Agnieszka

Naya, Francisco

Nazarov, Andrej P.

Nazarski, Ryszard B.

Nazzaro, Filomena

Ndieyira, Joseph

Neal, Luke

Nedachi, Taku

Nedialkov, Paraskev Todorov

Neelamegham, Sriram

Nees, Matthias

Negrete, George

Nehira, Tatsuo

Nehmé, Reine

Neilson, Andrew

Nejad, Mojgan

Nemzer, Boris

Nencka, Radim

Neng, Nuno

Nepal, Madhav

Neri, Placido

Nesterov, Dmytro

Nesterova, Irina $\mathrm{V}$.

Netto-Ferreira, Jose Carlos

Neugart, Susanne

Neuhaus, Jochen

Neumann, Heinz

Neumann, Terrence

Neunert, Grazyna

Nevárez-Moorillón, Guadalupe Virginia

Neves, Adriana Cunha

Newman, David J.

Newman, Stephen

$\mathrm{Ng}$, Tzi Bun

Ngai, Ming-Yu

Ngai, To

Ngamcherdtrakul, Worapol

Nguyen, Thanh Binh

Nguyen, Trieu

$\mathrm{Ni}$, Dalong

Nichols, David

Nicolás, Francisco E.

Nicoletti, Ferdinando

Nicoletti, Rosario

Nicosia, Aldo

Niederman, Robert A.

Niedziela, Tomasz

Niehaus, Eva-Maria

Niehaus, Tom

Nielsen, Lars 
Nielsen, Line Hagner

Nielsen, Vance G.

Nieman, David C.

Nieminen, Kaarlo

Niemirowicz-Laskowska, Katarzyna

Niidome, Takuro

Nikas, Spyros P.

Nikel, Pablo Ivan

Nikolaev, Viacheslav

Nikolakaki, Eleni

Nikolakakis, Ioannis

Nikolakopoulou, Angeliki M.

Nikolopoulos, D.

Nilghaz, Azadeh

Nilsson, Ake

Nilsson, Henrik

Nimalaratne, Chamila

Nishi, Kosuke

Nishida, Takehiro

Nishikitani, Yoshinori

Nishimoto, Yoshio

Nishiwaki, Hisashi

Nishiyama, Yusuke

Nisnevitch, Marina

Nissan, Joseph

Nistor, Cristina

Nitulescu, George Mihai

No, Joo Hwan

Nobili, Stefania

Nobre, Beatriz

Noda, Hidetoshi

Nogales-Bueno, Julio

Noguchi, Constance Tom

Norman, Trevor

Normand, Bernard

Norouzitallab, Parisa

Norval, Mary

Nouranian, Sasan

Novak, Lajos

Novikov, Alexander

Novikova, Nataliya E.

Novinec, Marko

Novio, Fernando

Novío, Silvia

Nowacka-Jechalke, Natalia

Nowak, Adriana

Nowak, Renata

Nowicka, Anna

Nowicka, Grażyna

Nowicki, Janusz

Ntie-Kang, Fidele

Ntougias, Spyridon
Nüesch, Frank

Nuhn, Lutz

Nuić, Ivona

Núñez, Oscar

Nurieva, Evgeniya

Nürnberg, Dennis

Nycz, Jacek E

O’Duill, Miriam L.

Obata, Yasuko

Obrador, Elena

Oda, Yukari

Odenthal, Margarete

O'Doherty, George

Offord, James

Ofir, Rivka

Ogasawara, Hirohito

Ogasawara, Toru

Ogawa, Akiya

Ogino, Toshio

Ognyanov, Manol

Ogórek, Rafał

Ogungbe, Ifedayo

Ogunjirin, Adebowale

Oh, Kyung Taek

Oh, Kyungsoo

Oh, Man-ho

Oh, Seikwan

Oh, Seung Jae

O'Hagan, David

Ohnishi, Masatoshi

Ohta, Shinji

Ohta, Yoshihiro

Ojala, Johanna

Ojeda-May, Pedro

Ojika, Makoto

Ojima, Koichi

Oka, Natsuhisa

Okada, Taketo

Okawara, Toru

Okazawa, Atsushi

O'Keefe, Barry

Okino, Tatsufumi

Okovytyy, Sergiy

Okuda, Masayuki

Okumura, Nobuaki

Okunade, Adewole L.

Olaru, Octavian Tudorel

Olatoye, Marcus O.

Olech, Marta

Olejar, Kenneth J.

Olejniczak, Iwona

Olejnik, Anna 
Olejnik, Malgorzata

Oleskin, Alexander V.

Oliva, Leone

Oliva, Romina

Oliveira, Beatriz

Oliveira, Hugo M.

Oliveira, Joana

Oliveira, Pedro

Oliveira, Rodrigo

Oliveira, Rui

Oliveira-Santos, Sonia Andreia

Oliveri, Paolo

Oliverio, Manuela

Oliver-Ortega, Helena

Oliviero, Giorgia

Olson, Kenneth

Omar, Syed Haris

Ombra, Maria Neve

Oms, Olivier

Onchoke, Kefa

Onduka, Toshimitsu

O'Neil, Gregory

Oniga, Ilioara

Oniszczuk, Anna

Onnis, Valentina

Onorato, Amber J.

Onuta, Marie-Christine

Onysko, Petro

Oon, Hazel H.

Opara, Elizabeth I.

Operamolla, Alessandra

Oracz, Joanna

O'Reilly, Elaine

Ori, Ottorino

Orian, Laura

Orlando, Tomas

Oroian, Mircea

Oroz-Guinea, Isabel

Orsi, Mario

Orte, Angel

Ortega, Angel L.

Orthaber, Andreas

Ortiz, Maria J.

Osborn, Helen

Osborne, James

Ośmiałowski, Borys

Osolodkin, Dmitry

Osório, Wislei R

Ostacolo, Carmine

Ostaszewski, Ryszard

Ostovar, Saeideh

Ostrowska, Kinga
Ostrowski, Stanisław

Otero, Carolina

Otero, Marta

Otmačić Ćurković, Helena

Otsuka, Masami

Otsuka, Yuzuru

Ott, Maria

Ou, Tian-miao

Owczarek, Aleksandra

Owen, Jeremy

Owusu-Apenten, Richard

Oyama, Dai

Ozaki, Toshinori

Ozawa, Shogo

Ozen, Mehmet Ozgun

Ozkan, Sveta Zhiraslanovna

Pace, Vittorio

Pachapur, Vinayak

Pacheco, Fabio Juliano

Pacheco, Neith

Pachuta-Stec, Anna

Paci, Maurizio

Pacureanu, Liliana

Padmanabhan, Jagannath

Padmanabhan, Parasuraman

Paduano, Antonello

Paduch, Roman

Paeng, Ki-Jung

Pagani, Stefania

Page, Melissa

Page, Richard C.

Paggetti, Jérôme

Pagnotta, Mario A.

Paixao Coelho, Jose Augusto

Pakulski, Zbigniew

Pal, Rumpa

Pal, Sudipto

Palacios, Oscar

Palacios, Sara

Palage, Mariana

Pałasz, Artur

Palenzuela López, José Antonio

Palermo, Edmund

Palinko, Istvan

Palla, Franco

Pallaoro, Alessia

Paller, Channing

Palma, Miguel

Palma, Vincenzo

Palmer, Andrew

Palomo, Jose

Palomo, Valle 
Palumbo, Carla

Pampana, Silvia

Pan, Guoqing

Pan, Linqiang

Pan, Xiaoyong

Pan, Y. J.

Pan, Yangang

Panagiotidis, Mihalis

Panaro, Maria Antonietta

Panay Escobar, Aram Joel

Panchal, Sunil K

Panderi, Irene

Pandey, Ramesh Prasad

Paneth, Agata

Paneth, Piotr

Panfoli, Isabella

Panniello, Annamaria

Pannkuk, Evan L.

Pantani, Roberto

Pantos, Dan

Panzella, Lucia

Paoli, Paola

Paoli, Paolo

Paolillo, Mayra

Paolo Busardó, Francesco

Paolone, Annalisa

Papadimitriou, Konstantinos

Papageorgis, Panagiotis

Papaioannou, Dionissios

Papakyriakou, Athanasios

Papamichael, Emmanuel

Papetti, Adele

Papini, Emanuele

Papo, Niv

Papoutsis, Konstantinos

Papp, András

Pappa, Aglaia

Pappas, Christos

Páramo-García, Ulises

Parashar, Deepak

Paraskevopoulou, Patrina

Parcells, Mark

Paredes, José Manuel

Parikesit, Arli Aditya

Parisini, Emilio

Park, Chung Gyoo

Park, Dae-Hun

Park, Dong Sun

Park, Enoch

Park, Hyung-Ho

Park, Il-Kwon

Park, Jeong Ho
Park, Jiyoung

Park, Jong-In

Park, Jong-Seung

Park, Joshua

Park, Ky Young

Park, Kyoung Chan

Park, Min-Jung

Park, Myung Hwan

Park, Simon

Park, Wansu

Park, Yongsoon

Parker, Stewart F.

Parlea, Lorena

Parolini, Cinzia

Parra, Alejandro

Parra, Roberto

Parrella, Edoardo

Parrilli, Annapaola

Pârvu, Alina Elena

Pascal, Simon

Pascali, Giancarlo

Pascarelli, Nicola Antonio

Páscoa, Ricardo

Paseiro-Cerrato, Rafael

Pasick, John

Pasini, Dario

Pasini, Luigi

Pasqua, Gabriella

Pasquale, Claudio De

Pasqualone, Antonella

Passamonti, Sabina

Passos, Fabiana

Pastore, Annalisa

Paszkiewicz, Sandra

Pátek, Miroslav

Patel, Alok

Patel, Anamika

Patel, Bhargav

Patel, Hardikkumar

Patel, Yashomati M.

Paterson, Brett

Pathakoti, Kavitha

Pathi, Suman

Patil, Avinash J.

Patra, Barunava

Patra, Jayanta Kumar

Patrauchan, Marianna A.

Patrizia, Nitti

Pattabiraman, Mahesh

Patterson, Adam V.

Patyra, Ewelina

Pauer, Werner 
Paul, Ananya

Pauliukaite, Rasa

Pau-Roblot, Corinne

Pavela, Roman

Pavic, Valentina

Pawelkowicz, Magdalena

Pawlicki, Miłosz

Payne, Annette

Payrastre, Bernard

Pazdro, Robert

Pazourek, Jiri

Peana, Massimiliano F

Peczuh, Mark

Pedatella, Silvana

Pedersen, Anders

Pedraza-Chaverri, Jose

Pedretti, Alessandro

Pedrosa, Mercedes M.

Pedrosa, Rafael

Pedroza, Mesias

Peeters, Marloes

Peifer, Christian

Peigneur, Steve

Pelagalli, Alessandra

Pelegov, Dmitry

Peleteiro, Mercedes

Pelin, Marco

Pellegrino, Sara

Pellei, Maura

Pellis, Alessandro

Pelosi, Claudia

Peluso, Ilaria

Pena-Pereira, Francisco

Peña-Ruiz, Tomás

Pence, Brandt

Penczek, Stanislaw

Pendleton, Phillip

Peng, Jun

Peng, Mengfei

Peng, Shu-Fen

Peng, Teng

Peng, Zhili

Penke, Botond

Pentak, Danuta

Pentzold, Stefan

Pepeu, Giancarlo

Pepi, Milva

Peppel, Tim

Peppelenbosch, Maikel

Perche, Fédérico

Perdih, Andrej

Perego, Carla
Pereira, David

Pereira, Eduardo

Pereira, Eliseu J.G.

Pereira, Florbela

Pereira, Frederico C.

Pereira, J.A.

Pereira, José Augusto

Pereira, Maria Do Carmo

Pereira, Olívia R.

Pereira, Vanda

Perestrelo, Rosa

Pérez Sestelo, José

Pérez, María J.

Perez, Serge

Pérez-Álvarez, Jose Angel

Perez-Jimenez, Jara

Perez-Stable, Carlos

Pérez-Villanueva, Jaime

Peri, Francesco

Perić, Mihaela

Perinelli, Diego Romano

Perlepes, Spyros

Permyakov, Eugene

Perna, Filippo

Perrakis, Anastassis

Perreault, Jonathan

Perteghella, Sara

Pertusati, Fabrizio

Peruzzi, Francesca

Pešić, Milica

Pestov, Alexandr

Peter, Emanuel K.

Peter, Katherine

PETER, Martin G.

Peterson, Julia

Peterson, Larryn

Petit, Patrice X.

Petkova, Nadezhda

Petrella, Andrea

Petriccione, Milena

Petrikaite, Vilma

Petrillo, Giovanni

Petropoulos, Spyridon

Petrov, Dmitry

Petrova, Krasimira T.

Petruczynik, Anna

Petruk, Ganna

Peyton, David H.

Pezzolla, Daniela

Pezzotti, Giuseppe

Pezzuto, Aldo

Pfeffer, Gerald 
Philips, Neena

Phuoc, Nghia Truong

Piao, Xijun

Piastowska-Ciesielska, Agnieszka Wanda

Piccialli, Vincenzo

Piccionello, Antonio Palumbo

Piccirillo, Clara

Pickova, Jana

Picmanova, Martina

Picone, Gianfranco

Pidatala, Venkata Ramana

Pielichowski, Krzysztof

Piemontese, Luca

Pieraccini, Stefano

Piergiovanni, Luciano

Pierpaoli, Elisa

Pierre, Guillaume

Pietrella, Donatella

Pietrzyk, Bozena

Pigge, F. Christopher

Pigłowski, Marcin

Pignitter, Marc

Pihlava, Juha-Matti

Pilkington, Lisa

Pilo-Pais, Mauricio

PINA, Fernando

Pina, João

Pincus, Seth

Pindi, Suresh

Pineda, Antonio

Pineda, Miguel

Piñeiro, Marta

Pinela, José

Pingault, Lise

Pinheiro, Marina

Pinnapireddy, Shashank Reddy

Pino, Manuel M. Sánchez Del

Pintea, Adela

Pinto Carvalho, Ana Maria

Pinto Reis, Catarina Pinto

Pinto, Diana C. G. A.

Pinto, Mafalda

Pinto, Milena

Pinto, Paula

Pinto, Uelinton

Pinu, Farhana R.

Pinzaru, Iulia

Piotrowska, Urszula

Piotrowski, Piotr

Piovesana, Susy

Pires, Regina Helena

Pirillo, Angela
Piringer, Martin

Piriou, Yannick

Piro, Gabriella

Pisano, Pablo L.

Pisarska, Joanna

Pisklak, Dariusz Maciej

Pissarek, Margit

Pitto, Letizia

Pitucha, Monika

Piva, Terrence

Pivetta, Tiziana

Pivonello, Rosario

Pizzi, Antonio

Pizzorno, Mario Andres

Plácido-Escobar, Jersson E.

Pla,der, Wojciech

Planas, Marta

Plano, Daniel

Plastina, Pierluigi

Plate, Manuela

Platts, James A.

Plavec, Janez

Plaza, Alberto

Plażuk, Damian

Plessas, Stavros

Plíhalová, Lucie

Plonka, Przemyslaw

Płotka-Wasylka, Justyna

Plotnikov, Egor

Pluquet, Olivier

Plutino, Maria Rosaria

Plyusnin, Victor F.

Poater, Albert

Poczai, Péter

Poddel'sky, Andrey

Pogorzelska, Aneta

Pogorzelska-Nowicka, Ewelina

Pohl, Pawel

Poinsot, Verena

Poitras, Daniel

Poku, Rosemary A.

Polakova, Monika

Polanski, Marek

Polavarapu, Lakshminarayana

Polavarapu, Prasad L.

Poletto, Matheus

Poli, Giulio

Poliak, Peter

Polischuk, Pavel

Polishchuk, P.

Politeo, Olivera

Pollmann, Katrin 
Pollock, Richard L.

Polo, Beatriz De Las Heras

Polyak, Steven

Polyakov, Nikolay

Poma, Paola

Pomerantz, William

Pomin, Vitor $\mathrm{H}$.

Pons, Josefina

Pons, Miquel

Pooler, Darcy

Poór, Miklós

Poozesh, Sadegh

Popa, Mona Elena

Popiołek, Łukasz

Poplawski, Tomasz

Popović-Djordjević, Jelena B

Popovich, David

Porcaro, Francesco

Porchia, Marina

Porciani, David

Porebski, Grzegorz

Portaro, Fernanda

Porto, André Luiz Meleiro

Potapov, Andrei

Potparević, Biljana

Potterat, Olivier

Poudel, Tej Narayan

Pouli, Nicole

Pountney, Dean

Poupot, Remy

Pourcet, Benoit

Pourquier, Philippe

Pourrahimi, Amir Masoud

Powell, Michael

Pozo, Óscar J.

Prajzler, Vaclav

Pranovich, Andrey

Prassl, Ruth

Pratsinis, Harris

Preite, Marcelo

Prenzler, Paul

Prescott, John F.

Preti, Delia

Priel, Avi

Prieto, Maria Pilar

Prieto-Lloret, Jesus

Prim, Damien

Prima, Giulia Di

Primožič, Ines

Prinsi, Bhakti

Prior, Sarah

Prisic, Sladjana
Prochazka, David

Proestos, Charalampos

Prohens, Jaime

Proietti, Silvia

Prokai, Laszlo

Prosen, Helena

Proserpio, Davide

Protti, Michele

Prudent, Michel

Pruess, Birgit M.

Pruvost, Alain

Przybylski, Piotr

Przybyt, Małgorzata

Psurski, Mateusz

$\mathrm{Pu}$, Jingzhi

Pucheault, Mathieu

Puglia, Debora

Puglisi, Rita

Puglisi-Weening, Melany

Puiggalí, J.

Puiggalí, Jordi

Puizina, Jasna

Punganuru, Surendra Reddy

Purchas, Roger W.

Puttreddy, Rakesh

Putz, Mihai V.

Py, Sandrine

Pyka-Pająk, Alina

Pytka, Karolina

Pyzik, Michal

Qaim, Syed M.

Qian, Pengxu

Qiao, Liang

Qin, Sanbo

Qiu, Huan

Quadrelli, Paolo

Quan, Yangjian

Quartu, Marina

Queiroz, Maria-Joao

Quesada Pérez, Víctor Manuel

Quiles, José L.

Quillard, Thibaut

Quinn, Mark T.

Quintard, Jean-Paul

Qvortrup, Katrine

R. Odell, Luke

R. Santos, Cleydson Breno

Raabe, Gerhard

Rabanal Anglada, Francesc

Rabbani, Gulam

Race, Paul

Rachek, Lyudmila 
Rachwalski, Michał

Rácz, Anita

Radchenko, Eugene V.

Radhakrishnan, Sivaprakasam

Rádis-Baptista, Gandhi

Radmila, Pavlovic

Radošević, Kristina

Radoshevich, Lilliana

Radu, Gabriel-Lucian

Radwan, Mohamed

Ragauskas, Arthur J.

Ragg, Enzio

Ragno, Gaetano

Ragno, Rino

Ragupathy, Viswanath

Ragusa, Andrea

Ragusa, Maria Antonietta

Rahimi, Farid

Rahman, Khondaker Miraz

Rahman, Khondokar Mizanur

Rahman, Masmudur M.

Rahman, Ziaur

RahmanPour, Rahman

Rahme, Kamil

Rai, Dilip

Rai, Prakash

Raimondi, Daniele

Raina, Medha

Raines, Ronald

Raingeaud, Joël

Rajauria, Gaurav

Rajca, Mariola

Rajčević, Nemanja

Raje, Mithun

Rajnarayanan, Rajendram

Rakitin, Oleg A.

Rakotondraibe, Harinantenaina Liva

Ralston, Emily

Ramachandran, Anup

Ramaiahgari, Sreenivasa C.

Ramakrishnan, Srinivasan

Ramalho, Teodorico

Ramanavicius, Arunas

Ramaraj, Pandurangan

Ramasamy, Elamparuthi

Rambaldi, Mirella

Ramesh, Sreerangappa

Ramil, Carlo

Ramirez-Vick, Jaime

Ramis, Gianguido

Ramona, Paltinean

Ramos, Pedro
Rana, Dipak

Ranc, Václav

Rangel-Hernandez, Victor

Rangnekar, Abhijit

Ranzato, Elia

Rao, Vidhya

Rapeanu, Gabriela

Raposo, Maria Filomena De Jesus

Rapposelli, Simona

Raptopoulos, Grigorios

Rascio, Agata

Rascon, Agustin

Rastija, Vesna

Ratajewski, Marcin

Rateb, Mostafa E.

Rathinasabapathy, Anandharajan

Ratkova, Ekaterina L.

Ratnayake, Ranjala

Raudino, Antonio

Raugei, Giovanni

Raupp, Sebastian M.

Rauwel, Protima

Ravegnini, Gloria

Ravelli, Davide

Ravenscroft, Neil

Raviola, Carlotta

Rawel, Harshadrai

Rayan, Anwar

Rayson, Gary D.

Reader, Jocelyn C.

Rebelo, Susana

Rebl, Alexander

Reboredo-Rodríguez, Patricia

Reboud, Julien

Rebros, Martin

Recabarren Gajardo, Gonzalo

Reddy, Sakamuri

Reetz, Manfred T.

Reeve, Vivienne

Regal, Patricia

Reginato, Mauricio J.

Reguero, Mar

Rehrig, Erin M.

Reich, Robert

Reichert, David E.

Reid, Christopher W.

Reina, Tomas Ramirez

Reinaud, Olivia

Reis, Celso

Reiss, Guido J.

Reissmann, Siegmund

Reiter, Russel 
Remaut, Katrien

Remesar, Xavier

Remize, Fabienne

Ren, Yulin

Renata, Hans

Renault, Nicolas

Renčiuk, Daniel

Rendle, Phillip M.

Renman, Gunno

Renner, Ulrich

Renzi, Polyssena

Rescifina, Antonio

Reshkin, Stephan J.

Revilla, Eugenio

Rey, Alejandro

Rey, Luis

Reyes, Fernando

Reyes-Chilpa, Ricardo

Reyes-Garcés, Nathaly

Reynisson, Jóhannes

Reynolds, Andrew

Rey-Stolle, Fernanda

Rezaei-Ghaleh, Nasrollah

Reznik, Sandra

Rezzani, Rita

Rhee, Hyun-Woo

Rhee, Jin-Kyu

Rho, Hoon Suk

Rho, Jung-Rae

Rhodes, Diana C. J.

Ribeiro, Claudia

Ribeiro, João

Rice, John

Ricelli, Alessandra

Richa, Tambi

Richard, John

Richard, Mandle

Richardson, Alan

Richomme, Pascal

Richter, Katrin

Richter, Shachar

Riedl, Rainer

Rienzo, Monica

Rigamonti, Luca

Rigano, Daniela

Rigano, Maria Manuela

Rigo, Eveline

Rijo, Patrícia

Rimondini, Lia

Rinnerthaler, Mark

Ríos-Gutiérrez, Mar

Rishi, Arun
Risoluti, Roberta

Rita Costa-Pinto, Ana

Ritala, Mikko

Ritch, Jamie

Ritzoulis, Christos

Rivas, Fatima

Rivera De La Rosa, Javier

Rivera-Monroy, Zuly Jenny

Rivero, Sonia

Rizvi, Syed

Rizzo, Antonio

Rizzolio, Flavio

Roach, Thomas

Robbennolt, Shauna

Robert, Eric

Roberts, Sue A.

Robertson, Avril

Robertson, Mark J.

Robiette, Raphael

Robinson, Sara

Robledo, Virginia Rodríguez

Robles Medina, Alfonso

Robles, Eduardo

Robson, Matthew

Rocca, Carmine

Roccatano, Danilo

Rocchetti, Gabriele

Rocchigiani, Luca

Rocha, Hugo A. O.

Rocha-Martin, Javier

Rochefort, Simone

Rocio-Bautista, Priscilla

Roderburg, Christoph

Rodrigues, António S.

Rodrigues, Francisca

Rodrigues, Joana

Rodrigues, Joao

Rodrigues, Olivier

Rodríguez García, Mario Enrique

Rodriguez Lorenzo, Luis Maria

Rodriguez, Hortensia M.

Rodriguez, Juan B.

Rodriguez, Noel

Rodríguez, Teresa Pineda

Rodriguez-Cueto, Carmen

Rodríguez-Gómez, Arturo

Rodríguez-Pérez, Celia

Rodríguez-Pérez, José Manuel

Rodríguez-Roque, María Janeth

Rodziewicz, Pawel

Roelen, Bernard

Roglans, Anna 
Rogozhin, Eugene

Roh, Changhyun

Roh, Jaroslav

Roh, Sangho

Rohs, Remo

Roilo, David

Roj, Edward

Rollin, Patrick

Rollini, Manuela

Román Guerrero, Angélica

Romanelli, Maria Novella

Romano, Nicla

Romanov, Georgy A.

Romanucci, Valeria

Romeo, Roberto

Romero, Aldo Humberto

Romero, Alejandro

Romero-Muñiz, Carlos

Romero-Salguero, Francisco J.

Rondeau-Gagné, Simon

Root, Martin

Rosa, Marcelo B. Da

Rosato, Antonio

Roşca, Sorin-Claudiu

Roscini, Luca

Rose, Peter.

Rosebrock, Adam P.

Roselló, Salvador

Rosencrantz, Ruben R.

Rosicka-Kaczmarek, Justyna

Rosini, Elena

Ross, Ian

Ross, Ian L

Rossi, Miriam

Rossignol, Julien

Rossiter, Sharon

Rosso, Natalia

Roszak, Szczepan

Roterman, Irena

Roth, Stephan V.

Rouhiainen, Ari

Roullier-Gall, Chloé

Roussel, Christian

Roussis, Vassilios

Routray, Winny

Roy Choudhury, Swarup

Roy, Dipankar

Roy, René

Roy, Sagar

Roychowdhury, Sanjoy

Różalska, Barbara

Rozalski, Marcin
Rozes, Nicolas

Rozhon, Wilfried

Rozumová, Lucia

Ruan, Ke

Rubin, Seth M.

Rubino, Federico Maria

Rubiolo, Patrizia

Rubis, Blazej

Rudat, Jens

Rudd, Sean G.

Ruddraraju, Kasi Viswanatharaju

Rudick, Jonathan

Rudnicka, Wiesława

Rudrawar, Santosh

Rudyak, Valery Ya.

Rudzińska, Magdalena

Ruelland, Eric

Ruggieri, Fabrizio

Rugnini, Lorenza

Rui, Yue

Ruiu, Luca

Ruiz Ramos, Encarnación

Ruiz Rubio, Leire

Ruiz, M. Pilar

Ruiz, Sergio

Rusinska-Roszak, Danuta

Rusnati, Marco

Russo, Gian Luigi

Russo, Maria

Russo, Mariateresa

Russo, Mario Vincenzo

Russo, Nino

Russu, Wade

Rusu, Aura

Ruthstein, Sharon

Rutjes, Floris P.J.T.

Ruzek, Daniel

Ruzik, Lena

Ruzza, Paolo

Ryan, Ali

Ryan, Barry

Ryan, Edward T.

Ryan, Michael P.

Rybarczyk, Piotr

Rybczyńska-Tkaczyk, Kamila

Rychtera, Mojmir

Ryl, Jacek

Ryu, Shi Yong

Rzepecka-Stojko, Anna

Rzymowska, Jolanta

Sá, Jacinto

Saad, Hosam 
Sabate, Raimon

Sabater I Serra, Roser

Sabatier, Jean-Marc

Sabella, Erika

Sabot, Cyrille

Sacchetti, Alessandro

Sacks, Gavin

Sackus, Algirdas

Sada, Kazuki

Sadowska, Beata

Saez, Carmen

Sagui, Celeste

Saha, Mahasweta

Sahi, Vaidurya Pratap

Sahoo, Pabitra Kumar

Saiano, Filippo

Saielli, Giacomo

Sainlos, Matthieu

Saisho, Yoshifumi

Saito, Genki

Saito, Yoshinori

Saito, Yoshiro

Saitoh, Kenji

Saiz-Fernández, Iñigo

Sakai, Atsushi

Sakai, Ryuichi

Sakamoto, Kensaku

Sakilam, Satish

Sakudo, Akikazu

Sakurai, Minoru

Sakurai, Toshihiro

Sala, Roberto

Salachna, Piotr

Salaheen, Serajus

Salahub, Dennis

Salas, Carlos

Salazar-Olivo, LA

Saldanha, Sabita

Salehin, Mohammad

Salehizadeh, Hossein

Salerno, Loredana

Sales, Carlos

Salim, Vonny

Salmerón, Ivan

Saluk-Bijak, Joanna

Salvador, J.-Pablo

Salvatore, Pepe

Salvo, Andrea

Samaja, Michele

Samanidou, Victoria F

Šamec, Dunja

Samek, Ota
Samková, Eva

Sampaio, Natalia G.

Sams, Thomas

Samuel, Temesgen

San Fabian, Emilio

Sana, Thibault G

Sancenón, Félix

Sanchez, Diego H.

Sánchez, Goar

Sánchez, María Teresa

Sánchez-Fidalgo, Susana

Sánchez-García, David

Sánchez-Martínez, Concha

Sanchez-Moreiras, Adela

Sancineto, Luca

Sanda, Takaomi

Sandasi, Maxleene

Sandjo, Louis Pergaud

Sándor, Gonda

Sandri, Brian J.

San-Félix, Ana Rosa

Sanganyado, Edmond

Sangiovanni, Enrico

Sani, Gabriele

Sanjust, Enrico

Sankaran, Ganapathy Subramanian

Sankaranarayanan, Nehru Viji

Sanmartín Grijalba, Carmen

Sanmartin, Chiara

Sanoh, Seigo

Sansone, Anna

Santacroce, Luigi

Santamaria, Rita

Santambrogio, Paolo

Santana, Andrés González

Santander-Jiménez, Sergio

Santangelo, Carmela

Santhakumar, Abishek

Santibanez-Lopez, Carlos E.

Santilio, Angela

Santini, Antonello

Santolla, Maria Francesca

Santoro, Fabrizio

Santos Júnior, Aníbal De Freitas

Santos, Clementina

Santos, Helder

Santos, João M.

Santos, Marcone Moreira

Santos, Maria M. M.

Santos-Gallego, Carlos

Santoso, Netty

Santulli, Gaetano 
Sanz Garcia, Andres

Sanz García, Juan

Saotome, Masao

Saoud, Khaled

Sapino, Simona

Sarabia, Francisco

Sardeli, Chrysanthi

Sarkar, Dhruba

Sarkar, Sujan

Sarma, Saurav

Sarti, Elena

Sartori, Luca

Sasai, Yasushi

Sasaki, Hideo

Sasaki, Kazuhiro

Sasaki, Motoko

Sasaki, Nobuhiro

Sashiwa, Hitoshi

Sassi, Mohamed

Sassone, Jenny

Satake, Masayuki

Satbhai, Santosh Balasaheb

Satheesh, Sathyaneson

Šatínský, Dalibor

Sato, Emiko

Sato, Hideyuki

Sato, Hisako

Sato, Kazuyuki

Sato, Shin

Satou, Tadaaki

Saturnino, Carmela

Satyal, Prabodh

Saucier, Cedric

Saurav, Kumar

Saurina, Javier

Savage, Paul B.

Savastano, Silvia

Savi, Monia

Saville, Dorothy

Saviola, Anthony

Savion, Naphtali

Savoie, Jean-Michel

Savy, Davide

Sawai, Hirofumi

Sawicki, Rafal

Sayre, Casey L.

Sbardella, Gianluca

Scampicchio, Matteo

Scarafoni, Alessio

Scarfato, Paola

Scarselli, Marco

Scarso, Alessandro
Scendo, Mieczyslaw

Schaefer, Jennifer

Schaefer, Jeremy S

Schallmey, Anett

Schang, Luis

Scheicher, Ralph H.

Scheiner, Steve

Schenk, Michael

Schepmann, Dirk

Schiavone, Marco

Schild-Poulter, Caroline

Schillaci, Domenico

Schiraldi, David A.

Schirhagl, Romana

Schirmer, Bastian

Schlueter, Klaus-Dieter

Schmeda-Hirschmann, Guillermo

Schmid, Jochen

Schmidt, Bernhard

Schmidtke, Michaela

Schmitz, John C.

Schnackenberg, Laura

Schneekloth, John

Schneider, Sabine

Schomburg, Lutz

Schrittwieser, Stefan

Schroeder, Grzegorz

Schuehly, Wolfgang

Schulz, Benjamin L.

Schulz, Florian

Schulze, Johannes

Schulze, Margit

Schulzke, Carola

Schuman, Meredith C.

Schurgers, Leon J.

Schurig, Volker

Schwalbe, Carl H.

Schwaminger, Sebastian

Schwan, Marina

Schwan, William

Schwartz, Andrew J.

Schwarze, Michael

Schweigkofler, Wolfgang

Schweizer, Thomas

Schwiebs, Anja

Schwikkard, Sianne

Ścianowski, Jacek

Ścibisz, Iwona

Scicchitano, Pietro

Scognamiglio, Monica

Scorziello, Antonella

Scott B., Halstead 
Scotti, Marcus

Scotti, Nunzia

Scotto D'Abusco, Anna

Seah, Stephen Y. K.

Seantier, Bastien

Sebastià, Natividad

Sebastiana, Mónica

Sebestik, Jaroslav

Seca, Ana

Seca, Ana Maria Loureiro Da

Sechi, GianPietro

Sedic, Mirela

See, Hong Heng

Segarra-Marti, Javier

Segura, Joan

Šegvić Klarić, Maja

Seidel, Rüdiger W.

Seidel, Thorsten

Seiersten, Marion

Seijas Vázquez, Julio

Seiler, Magdalene J.

Seiner, Brienne N.

Seiquer, Isabel

Sękowski, Szymon

Seley-Radtke, Katherine

Sellayah, Dyan

Selleri, Silvia

Selvam, Chelliah

Selyanchyn, Roman

Semenyuk, Pavel

Semeril, David

Sen, Sanghamitra

Senbonmatsu, Takaaki

Sendker, Jandirk

Senesi, Pamela

Sengupta, Bidisha

Seok, Hyun

Separovic, Frances

Septembre-Malaterre, Axelle

Sergi, Consolato

Serpersu, Engin H.

Serpico, Rosario

Serra, Stefano

Serradji, Nawal

Serralheiro, Maria Luísa

Serrano, Maria

Sestili, Sara

Sethi, Gautam

Seto, Shigeki

Setzer, William N.

Sevcovicova, Andrea

Seweryn, Artur
Sgherri, Cristina

Sgorbini, Barbara

Sgrignani, Jacopo

Shafiei, Ali

Shah, Manish

Shahidi, Fereidoon

Shang, Zhuo

Shankar, Eswar

Shanker, Anil

Shao, Fangwei

Shao, Huaiyu

Shao, Yihan

Sharma, Bhavya

Sharma, Bhesh

Sharma, Deepika

Sharma, Ritin

Sharma, Rohit

Sharma, Sanjiv

Sharman, Edward H.

Sharp, Jo

Shaughnessy, Kevin

Shavandi, Amin

Shavrukov, Yuri

Shaw, Odette

Sheardy, Richard

Shearer, Martin

Shehu, Amarda

Sheldon, Julie

Shen, Guofang

Shen, Szu-Chuan

Shen, Tongye

Shenderovich, Ilya G.

Sheng, Ruilong

Sheppard, Tom D.

Sherer, Nathan

Sheri, Madhu

Sherwood, James

Shestopalov, Alexander M

Shewale, Swapnil

Shi, Da-Yong

Shi, Junfeng

Shi, Suan

Shi, Wenbo

Shi, Xiaodong

Shi, Xiaoyang

Shi, Xinghua

Shi, Yi

Shi, Zhenzhen

Shiao, Young-Ji

Shibata, Norio

Shiea, Jentaie

Shieh, Tzong-Ming 
Shigdar, Sarah

Shih, Ming C.

Shiina, Takashi

Shikov, Alexander N.

Shil, Suranjan

Shim, Min Suk

Shimada, Masako

Shimada, Yasuhito

Shimizu, Kuniyoshi

Shimodaira, Shigetaka

Shimosawa, Tatsuo

Shin, Beom Soo

Shin, Dongyun

Shin, In-Sik

Shin, Jae Sup

Shin, Kwang-Hee

Shin, Young G.

Shinada, Tetsuro

Shinde, Sudhirkumar

Shinde, Suhas

Shinichi, Sekine

Shinomiya, Kazufusa

Shiragami, Tsutomu

Shirahata, Tatsuya

Shiraishi, Takehiko

Shirakami, Yohei

Shishido, Tânia K.

Shityakov, Sergey

Shoenfeld, Yehuda

Shoji, Tsubasa

Sholkamy, Eman

Shomura, Yasuhito

Shrimal, Shiteshu

Shubina, Elena

Shuhei, Nasuda

Shukla, Hem

Shul'pin, Georgiy B.

Shults, Elvira E.

Shumskaya, Maria

Siano, Francesco

Siatka, Tomáš

Siczek, Miłosz

Sidorenko, Viktoriya S.

Siebenhofer, Matthäus

Sieber, Fritz

Siemer, Ansgar B.

Sienkiewicz, Monika

Sier, Cornelis F.M.

Siewert, Bianka

Sigusch, Bernd W.

Sikazwe, Donald

Sikirzhytski, Vitali
Silaghi-Dumitrescu, Radu

Silchenko, Alexandra S.

Šiler, Branislav

Silina, Yuliya

Silva, Amelia M

Silva, Andre

Silva, Artur M. S.

Silva, Carlos Alberto

Silva, Dina M.

Silva, José

Silva, Luís

Silva, Luís R.

Silva, Márcio S.

Silva, Margarida F. B.

Silva, Maria Fátima

Silva, Pedro Jorge

Silva, Robson R.

Silva, Susana N.

Silva, Vera L. M.

Silvagno, Francesca

Silvestre, Samuel

Silvestri, Romano

Sim, Valerie

Simal-Gandara, Jesus

Simão, Daniel

Simari, Cataldo

Simeonov, Ivan

Simeonov, Vasil D.

Simha Martynkova, Grazyna

Simirgiotis, Mario

Simitzis, Panayiotis

Simka, Wojciech

Šimko, Fedor

Simmons, Katie

Simocko, Chester

Simoes, Mario

Simões, Marta Filipa

Simon, Julian

Simón, Luis

Simone, Angela De

Simone, Elena

Simonneaux, Gerard

Simpson, T. J.

Sims, Ian M.

Singh, Alok

Singh, Arunima

Singh, Chanpreet

Singh, Jitendra Kumar

Singh, Jugpreet

Singh, Kamalendra

Singh, Khushwant

Singh, Sarbjit 
Singh, Sasha

Singh, Somnath

Singh, Surya P.

Singha, Subhankar

Singhrao, Sim K.

Sinha, Abhijeet

Sinha, Birandra K.

Sinha, Jasmine

Sinha, Kalyan

Sinha, Sudarson

Sintim, Herman

Sinyukov, Alexander

Sipos, László

Sippl, Wolfgang

Sipponen, Mika Henrikki

Sips, Patrick

Siracusa, Laura

Sirimulla, Suman

Sita, Giulia

Sitarek, Przemysław

Sivaev, Igor B.

Sivakumar, Sushama

Sivasubramanian, Aravind

Sivieri, Katia

Siwek, Agata

Sizochenko, Natalia

Skalicka-Woźniak, Krystyna

Skalický, Milan

Skaltsa, Helen D.

Skandalis, Nikolaos

Skellam, Elizabeth

Skenderi, Katerina P

Skiba, Mohamed

Skorik, Yury A.

Skretas, Georgios

Skroza, Danijela

Skwarło-Sónta, Krystyna

Slavik, Roger

Slipchenko, Lyudmila

Ślipiko, Monika

Sliwiak, Joanna

Śliwińska-Wilczewska, Sylwia

Śliwiński, Tomasz

Śliwka-Kaszyńska, Magdalena

Słoczyńska, Karolina

Slominski, Andrzej

Slosarczyk, Agnieszka

Sluchanko, Nikolai

Slusarenko, Alan

Smani, Younes

Smaoui, Slim

Šmejkal, Karel
Smeriglio, Antonella

Smetana, Karel

Smetana, Michael

Smid, Scott D.

Smiesko, Martin

Śmiga-Matuszowicz, Monika

Śmigielski, Krzysztof B.

Smith, Mark

Smith, Matthew

Smith, Micholas Dean

Smith, Robert A.

Smith, Terry

Smout, Michael

Smrke, Samo

Smułek, Wojciech

Smyrniotopoulos, Vangelis

Snegur, Lubov

Snežana, Papović

Snowden, Timothy

Sobeh, Mansour

Sobiech, Monika

Sobisch, Titus

Sobkowski, Michal

Sobotta, Łukasz

Sobral, Abilio J.F.N.

Socaci, Sonia

Soejima, Tetsuro

Soengas, Raquel

Soengas, Raquel G.

Soica, Codruta

Sokół-Łętowska, Anna

Sokolov, Maxim

Šolaja, Bogdan A.

Solano, Francisco

Solcan, Carmen

Solel, Ephrath

Soleymani Abyaneh, Hoda

Soliman, Mahmoud E

Soliven, Arianne

Sollogoub, Matthieu

Solomon, Melani

Solomonov, Boris

Soloshonok, Vadim A.

Solovyev, Andrey

Somani, Sukrut

Sommer, Stephan

Son, Jaekyoung

Song, Chunhua

Song, Guoqing

Song, Imsook

Song, Jinhua

Song, Kwang Ho 
Song, Kyung Bin

Song, Mei

Song, Sang Hoon

Song, Tao

Song, $\mathrm{Xu}$

Song, Yang-heon

Sopade, Peter A.

Šoral, Michal

Sorg, Olivier

Sortais, Jean-Baptiste

Sosna, Justyna

Soto-Hernández, Marcos

Soucy, Shannon

Soulère, Laurent

Soural, Miroslav

Sousa, Ana

Souza-Smith, Flavia

Sovova, Helena

Sowa, Ireneusz

Spanakis, Marios

Spano, Giuseppe

Spanopoulos, Ioannis

Sparacino-Watkins, Courtney

Speciale, Antonio

Spee, Bart

Spégel, Peter

Spinella, Aldo

Spinelli, Laura

Spingler, Bernhard

Spivak, David

Spohr, Eckhard

Spradley, Frank T.

Springer, Lindsay

Sprio, Andrea Elio

Spur, Bernd W.

Spyros, Gkelis

Sreedasyam, Avinash

Sreerama, Subramanya

Sridhar, Jayalakshmi

Srihari, Sriganesh

Srinivas, Keerthi

Sriram, Gopu

Srivastava, Atul

Srivedavyasasri, Radhakrishnan

Srivenugopal, Kalkunte

Sroka, Zbigniew

Staal, Jens

Stacchiotti, Alessandra

Stadler, Marc

Stadnik, Joanna

Stagos, Dimitrios

Staniszewska, Monika
Stankevic, Marek

Stanovnik, Branko

Starostik, Petr

Starowicz, Małgorzata

Starzak, Karolina

Stasiuk, Graeme

Stassen, Ivo

Staszak, Katarzyna

Stavrianidi, Andrey

Stecca, Barbara

Steck, Todd R.

Steed, Jonathan

Steele, Terry W.J.

Stefane, Bogdan

Stefanowicz-Hajduk, Justyna

Stefanuto, Pierre-Hugues

Stefek, Milan

Steibliene, Vesta

Stein, Matthias

Steinfeldt, Norbert

Steinmann, Casper

Stenstrøm, Yngve

Stepanovic, Stepan

Stephan, Holger

Stephen, Michael Rajesh

Stephens, Jacqueline M.

Stępień, Łukasz

Steup, Martin

Stevens, Cole

Stevens, Erland

Stevenson, Bradley

Steverding, Dietmar

Stieger, Bruno

Stilli, Donatella

Stipanovic, Arthur

Stiriba, Salah-Eddine

Stjepanović, Marija

Stobiecka, Magdalena

Stobiecki, Maciej

Stöckelhuber, Klaus Werner

Stockmann, Christian

Stoikov, Ivan Ivanovich

Stojakowska, Anna

Stojan, Jure

Stojko, Jerzy

Stoleru, Elena

Stone, Kari

Stonik, Valentin A.

Stornaiuolo, Mariano

Stortini, Angela Maria

Stratford, Robert E.

Strati, Irini F. 
Stratmann, Bernd Straus, Suzana K. Strauss, Phyllis Strehmel, Veronika Strekowski, Lucjan Streubel, Robert Stroffekova, Katarina Strube, Jochen Struga, Marta Strzelecki, Michal Strzemiecka, Beata Stuart, David Studzińska-Sroka, Elżbieta Stuper-Szablewska, Kinga Sturm, Sonja Stürup, Stefan

Su, Chunming

$\mathrm{Su}$, Jui-Hsin

Su, Meng

Su, Peifeng

Subramaniam, Prasad

Sucheck, Steven J.

Suda, Yoshihito

Sugahara, Takuya

Sugai, Takeshi

Sugawara, Akihiro

Sugimoto, Yukio

Sugimura, Haruhiko

Sugiura, Yoshimasa

Suh, Dae-yeon

Suhail, Yasir

Sukhikh, Taisiya

Sukumaran, Anil

Sukumaran, Sunitha

Suleria, Hafiz

Suliburska, Joanna

Sulman, Esther

Sulová, Zdenka

Sun, Chen

Sun, Dianqing

Sun, Fengjie

Sun, Guohui

Sun, Hao

Sun, Jingjing

Sun, Lina

Sun, Litao

Sun, Meng

Sun, Shi

Sun, Shih-Jye

Sun, Wei

Sun, Wujin

Sun, Xinghui
Sun, Yingjie

Sunami, Yoshiaki

Sunatsuki, Yukinari

Sundheim, Leif

Sung, Ping-Jyun

Sung, Wang Chou

Sung, Wen-Chieh

Suravajhala, Prashanth

Suresh, Kuthuru

Surguchov, Andrei

Surma, Magdalena

Surmenev, Roman A.

Surmick, David

Surup, Frank

Suryawanshi, Vasantika

Susic, Michael

Sutherland, John B.

Sutton, Peter W.

Suzuka, Toshimasa

Suzuki, Katsuhiko

Suzuki, Kazumasa

Suzuki, Shigekatsu

Suzuki, Toshisada

Suzurikawa, Jun

Svärd, Michael

Švecová, Blanka

Sveda, Maria

Svetashev, Vasily

Svete, Jurij

Svingen, Terje

Svob Strac, Dubravka

Svoronos, Paris D.N.

Swarnkar, Gaurav

Swevers, Luc

Świątek, Łukasz

Świątek, Piotr

Świderska-Dąbrowska, Renata

Swierczynski, Julian

Swiezewska, Ewa

Sydnes, Magne O.

Syed, Viqar

Sylwester, Slusarczyk

Synytsya, Andriy

Syrpas, Michail

Szaciłowski, Konrad

Szacon, Elzbieta

Szafraniec, Joanna

Szafrańska, Katarzyna

Szafranski, Krzysztof

Szakonyi, Zsolt

Szaleniec, Maciej

Szczolko, Wojciech 
Szekalska, Marta

Szekely, Gyorgy

Szeleszczuk, Łukasz

Szewczyk, Katarzyna

Szewczyk, Roman

Szikra, Dezső

Szilágyi, András

Szilvási, Tibor

Szklarczyk, Damian

Szkudelska, Tomasz

Szőri, Milán

Szostak, Michal

Sztanke, Małgorzata

Szumny, Antoni

Szumny, Antoni Jacek

Szweda, Piotr

Szymanowska, Urszula

Szymańska, Emilia

Szymański, Pawel

Ta, Hang

Tabarrini, Oriana

Tabarsa, Mehdi

Tabata, Makoto

Tacconelli, Stefania

Tadanaga, Kiyoharu

Tadashi, Watabe

Taddei, Maurizio

Tadepalli, Sirimuvva

Tadic, Vanja

Tagliatesta, Pietro

Taguchi, Y-h.

Tahir, Muhammad N

Tai, Dar-Fu

Taïeb, David

Taira, Junsei

Tajber, Lidia

Takabe, Wakako

Takada, Tomoya

Takagi, Yasumasa

Takahama, Umeo

Takahashi, Daisuke

Takahashi, Fumiki

Takahashi, Kaito

Takahashi, Kosaku

Takahashi, Ohgi

Takahashi, Osamu

Takahashi, Shouji

Takahashi, Taku

Takahashi, Toshio

Takanami, Toshikatsu

Takano, Katsura

Takayama, Yoshiharu
Takayanagi, Toshiyuki

Takayuki, Ban

Takebayashi, Shin-ichiro

Takeda, Youhei

Takemori, Hiroshi

Takemoto, Hiroyasu

Takemoto, Masumi

Takemura, Miho

Takenaka, Shinji

Takeshita, Haruo

Takeuchi, Daisuke

Takizawa, Shinobu

Takó, Miklós

Takulder, Poulami

Talele, Tanaji $\mathrm{T}$

Talhi, Oualid

Talora, Claudio

Taly, Antoine

Tam, Eric Kwok Wai

Tamai, Ikumi

Tamama, Kenichi

Tamm, Toomas

Tamminen, Tarja

Tampucci, Silvia

Tamura, Osamu

Tan, Howe-Siang

Tan, Ming-Qian

Tan, Zhongping

Tanabe, Katsuyuki

Tanaka, Hiroyuki

Tanaka, Kazuo

Tanaka, Naonobu

Tanaka, Nobuyuki

Tanaka, Shigenori

Tanaka, Shinji

Tanaka, Takayuki

Tanaka, Tomonari

Tanase, Corneliu

Tanemura, Kiyoshi

Tang, Houliang

Tang, Longteng

Tani, Fumito

Tani, Hiroumi

Taniguchi, Nobukazu

Taniguchi, Tohru

Taniguchi, Yasushi

Tanimoto, Hiroki

Tankiewicz, Maciej

Tanner, Eden

Tanner, Julian A.

Tantillo, Dean

Tao, Lin 
Tao, Peng

Tapia, Alejandro A.

Tarantino, Giovanni

Tarman, Kustiariyah

Tarozzi, Andrea

Tashima, Kimihito

Tashima, Toshihiko

Tashiro, Etsu

Tashiro, Hirotaka

Tasic, Ljubica

Tasmin, Saira

Tate, Rothwelle J.

Tatikolov, Aleksander S.

Tatsumi, Yuki

Taube, Joe

Taura, Futoshi

Tava, Aldo

Tavares, Anthony

Tavares-da-Silva, Elisiario José

Tayebati, Seyed Khosrow

Taylor, Caroline

Taylor, Martin

Taylor, Matthew

Taylor, Maureen E.

Tcherdyntsev, Victor V.

Tecce, Mario Felice

Tedesco, Idolo

Teeri, Teemu

Teixeira Tomaz, Cândida

Teixeira, Róbson

Telegdi, Judit

Tellez, Trinidad Ruíz

Telukutla, Srinivasa Reddy

Telysheva, Galina

Tembrock, Luke R.

Tempone, André

Teng, Bo

Teng, Yong

Tengfei, Ji

Teodorescu, Florina

Teodoro, João

Tepe, Jetze J.

Terčelj, Marjeta

Terent'ev, Alexander O.

Terenzi, Alessio

Terpou, Antonia

Terry, Randall G.

Terzaghi, William

Terzaghi, William Bryan

Teschke, Rolf

Tetlow, Ian J.

Teusch, Nicole
Teymourian, Hazhir

Thakkar, Balmukund

Thakur, Vijay Kumar

Thamattoor, Dasan M.

Thapar, Roopa

Thiéry, Valérie

Thilmony, Roger

Thirunavukarasu, Deepak

Thiruvengadam, Muthu

Thissen, Peter

Thomas, Eric J.

Thomas, Eric Jim

Thomas, Joice

Thomas, Paul M.

Thomas, Samuel

Thomas, T.J.

Thomas, Y. K. Chan

Thomson, Neil H.

Threadgill, Michael

Thyagarajan, Baskaran

Tiera, Marcio

Tietjen, Ian

Tietze, Alesia

Tiffen, Jessamy C.

Tilley, Michael

Timón, Vicente

Timoshenko, Alexander V.

Timperio, Anna Maria

Ting, Richard C. H.

Tiruveedhula, V. V. N. Phani Babu

Tischler, Dirk

Tithi, Jesmin Jahan

Tiu, Brylee David

Tiwari, Amit

Tiwari, Rakesh K.

Tiwari, Vaibhav

To, Kin-Ying

Tobrman, Tomáš

Tocmo, Restituto

Toczylowska-Maminska, Renata

Todde, Sergio

Todea, Anamaria

Toiu, Anca

Tojo, Concha

Tolosa, Ezequiel

Toma, Lucio

Tomalia, Donald

Tomalia, Donald A.

Tomar, Dhanendra

Tomašič, Tihomir

Tomaszewska, Ewa

Tomczyk, Michał 
Tomczykowa, Monika

Tomi, Félix

Tomoda, Hiroshi

Tomuta, Ioan

Tondi, Gianluca

Tonelli, Michele

Tonolo, Giancarlo

Topic, Filip

Tori, Motoo

Toribio, Laura

Torigoe, Kanjiro

Torikai, Kohei

Tormo, José Rubén

Torre, Maria Luisa

Torres Lagares, Daniel

Torres Perales, Carolina

Torres Vaamonde, Jose Enrique

Torres, Jaume

Torres, Sara Sopeña

Torres-Chávez, Patricia I.

Torroba, Tomás

Tortorella, Domenico

Tósaki, Árpád

Toscani, Anita

Tosheva, Lubomira

Toshio, Koizumi

Tosi, Simone

Tosoni, Sergio

Tóth, Blanka

Tóth, Gergő

Totsingan, Filbert

Totta, Pierangela

Totti, Federico

Touil-Boukoffa, Chafia

Toy, Randall

Trabace, Luigia

Trabocchi, Andrea

Trainor, Patrick

Trammell, Scott A.

Tramontano, Enzo

Tran, Fabien

Tran, Phuong

Trandafirescu, Cristina

Tranfo, Giovanna

Trant, John

Travers, Jeffrey Bryant

Traweger, Andreas

Trentini, Alessandro

Trépout, Sylvain

Trevisan, Andrea

Tribe, Lorena

Triebl, Alexander
Trif, Monica

Trinchera, Marco

Trincone, Antonio

Trindle, Carl

Tripathi, Ashootosh

Tripathi, Lokesh P.

Tripathi, Manish

Tripodi, Gianluca

Tripodo, Giuseppe

Tripp, Carl P.

Tríska, Jan

Trivedi, Evan R.

Troise, Antonio Dario

Trojanowicz, Bogusz

Trombetta, Domenico

Trotsko, Nazar

Trougakos, Ioannis

Trovato, Fabio

Truhlar, Donald G.

Trujillo, Antonio-José

Trujillo, Cristina

Trzeciak, Anna M.

Tsafantakis, Nikolaos

Tsai, Ang-Chen

Tsai, Teh-hua

Tsai, Yenling

Tsaltas, Dimitrios

Tsapogas, Panagiotis

Tsay, Chien-Yie

Tse, William K.F.

Tseng, Chih-Hua

Tseng, Wei-Lung

Tsirigos, Konstantinos

Tsitsilonis, Ourania E.

Tsoi, James

Tsopmo, Apollinaire

Tsotinis, Andrew

Tsubaki, Kazunori

Tsuchida, Sachio

Tsuchimoto, Teruhisa

Tsuchiya, Kousuke

Tsuji, Atsushi B.

Tsuji, Hiroaki

Tsujibo, Hiroshi

Tsukamoto, Masaki

Tsunoda, Makoto

Tsuruoka, Takaaki

Tsutsui, Shigeyuki

Tsutsumi, Osamu

Tsyganenko, Alexey A.

Tsyshevskiy, Roman

$\mathrm{Tu}$, Min 
Tuccinardi, Tiziano

Tucker, Steven

Tudela, José

Tuenter, Emmy

Tueros, Itziar

Tulkens, Paul

Tullai, John

Tullio, Vivian

Tullius Scotti, Marcus

Tunaru, Sorin

Tunesi, Marta

Tuominen, Anu

Tupally, Karnaker R.

Turano, Paola

Turchin, Ilya

Turčić, Petra

Turco, Rosa

Turel, Iztok

Turel, Matejka

Turk, Tom

Turkman, Nashaat

Turkowski, Volodymyr

Turrini, Eleonora

Tuti, Simonetta

Tuttolomondo, Antonino

Tuvdendorj, Demidmaa

Tuzimski, Tomasz

Tvedt, Tor Henrik Anderson

Tvrdeic, Ante

Tvrdik, Petr

Tyagi, Rahul

Tzakos, Andreas G.

Tzen, Jason $\mathrm{T}$.

Tzounis, Lazaros

Tzschucke, Christoph

Uccelli, Licia

Uccello-Barretta, Gloria

Uchio, Yuji

üchncü, Muhammed

Uddin, Shaikh

Udumula, Venkata Reddy

Ueda, Hiroshi

Ueda, Mitsuhiro

Uehara, Tomoya

Uekusa, Hidehiro

Ueng, Yune-Fang

Ueno, Takafumi

Ueta, Ikuo

Ufnal, Marcin

Uhde, Erik

Uhde-Stone, Claudia

Uitdehaag, Joost
Uivarosi, Valentina

Ujor, Victor C.

Ulanski, Piotr

Ulasov, Ilya

Uludag, Hasan

Umemori, Juzoh

Umerska, Anita

Umeyama, Akemi

Umezawa, Taiki

Umoren, Saviour

Uneyama, Takashi

Unno, Keiko

Uno, Hidemitsu

Uno, Tomohide

Unsworth, Will

Unterlass, Miriam M.

Unterreiner, Andreas-Neil

Unudurthi, Sathya Dev

Upadhyay, Rakesh K.

Urashima, Tadasu

Urban, Philippe

Urbanczyk-Lipkowska, Zofia

Urbano, Ana Margarida

Urra, Felix

Usami, Yoshihide

Usui, Kenji

Usuki, Toyonobu

Usuki, Yoshinosuke

Uto, Yoshikazu

Uygun, Sahra

Uziel, Orit

V.G., Bessergenev

Vaca, Inmaculada

Vagner, Josef

Vaidya, Amita

Vaidyanathan, Bharat

Vaidyanathan, Ganesan

Vajda, Sandor

Valdes, Esperanza

Valencia, Gregorio

Valentao, Patricia

Valente, Sergio

Valenti, Maria Teresa

Valentine, Rudy J.

Valentová, Kateřina

Valenzuela, Hector

Valenzuela, Rodrigo

Valero, Marta Sofía

Valgimigli, Luca

Valles, Soraya L.

Vallet, Valérie

Valletta, Alessio 
Vallini, Giovanni

Valoppi, Fabio

Vamanu, Emanuel

Van Ballegooijen, Hanne

Van Bocxlaer, Katrien

Van Cruchten, Steven

Van Dam, Jan E.G.

Van Dam, R. Michael

Van De Sluis, A.J.A. (Bart)

Van Den Bergh, Hubert

Van Der Kuyl, Antoinette

Van Der Meulen, Nicholas P.

Van Dyke, Mark E.

Van Ee, Benjamin W.

Van Griensven, Leo

Van Herwijnen, Hendrikus W. G.

Van Horn, Wade

Van Ree, Teunis

Van Rijn, Richard M.

Vanderlei, Maria De Fátima

Vanelle, Patrice

Vann, Willie

Vannini, Andrea

Vanoni, Maria Antonietta

Ványolós, Attila

Váradi, Györgyi

Váradi, Judit

Varadwaj, Pradeep R.

Varaprasad, Kokkarachedu

Vareda, João

Varga, Elisabeth

Varga, Zoltan

Varoni, Elena Maria

Varrot, Annabelle

Varvounis, George

Vasaikar, Suhas

Vasas, Andrea

Vasicek, Ondrej

Vasile, Francesca

Vasiljevic, Todor

Vasilyev, Aleksander

Vasin, Mikhail V.

Vasko, Petra

Vassalle, Cristina

Vassilev, Nikolay G.

Vassilopoulos, George

Vaughan, Martha M

Vautier, Simon

Vavitsas, Konstantinos

Vaz Junior, Silvio

Vaz, Josiana

Vazquez Belda, Beatriz
Vázquez Tato, M. Pilar

Vázquez, José Antonio

Vazquez, Santi

Vazquez-Martínez, José Alfredo

Vecino, Xanel

Vega Gutierrez, Sarath

Vejux, Anne

Velasco-Vélez, Juan Jesús

Veldhuizen, Edwin

Veleeparambil, Manoj

Velena, A.

Velez, Zélia

Velkov, Tony

Vella, Laura Jayne

Vemula, Harika

Venditti, Alessandro

Venkatraman, Vishwesh

Vennerstrom, Jonathan

Venturi, Francesca

Venus, Joachim

Vereecken, Luc

Verestiuc, Liliana

Vergara, Daniele

Verhage, Leonie

Verhoeven, Adrie

Verma, Pragya

Verma, Rajni

Vermaas, Joshua V.

Vermeir, Lien

Vermeulen, Christiaan

Verotta, Luisella

Verweij, Hendrik

Vetere, Amedeo

Vetter, Stefan W.

Vetvicka, Vaclav

Vezzu, Dileep

Viapiana, Agnieszka

Viard, Mathias

Vicas, Simona

Vicente, Manuel Sastre De

Vichapong, Jitlada

Victor, Bruno

Vig, Komal

Víglaský, Viktor

Viglianti, Benjamin

Vigneron, Aurelien

Vila, Carlos

Vilar, Jose

Vilela, Alice

Villano, Debora

Villaverde, Juan José

Vincentini, Olimpia 
Vinciguerra, Manlio

Vining, Kelly

Virjamo, Virpi

Virzonis, Darius

Visioli, Francesco

Viso, Alma

Vistoli, Giulio

Vitalini, Sara

Vitalone, Annabella

Vladimira, Tomeckova

Vladimir-Knežević, Sanda

Vlamos, Panayiotis

Vlashi, Erina

Vo, Duc-Thang

Vodnar, Dan Cristian

Vogel, Alexander

Voglmeir, Josef

Vogt, Martin

Voiniciuc, Cătălin

Volcho, Konstantin P.

Voliani, Valerio

Volk, David

Volny, Michael

Volonterio, Alessandro

Volpe, Maria Grazia

Von Knethen, Andreas

Vondrasek, Jiri

Vorob'ev, Mikhail M.

Vougogiannopoulou, Konstantina

Voutchkova-Kostal, Adelina

Voziyan, Paul

Vranes, Milan

Vrecl, Milka

Vriend, Jerry

Vrontaki, Eleni

Vrsaljko, Domagoj

Vuković, Rosemary

Vullo, Daniela

Vuong, Thu V.

Vuorela, Heikki

Vyazovkin, Sergey

Vynios, Demitrios

Vyviurska, Olga

Wabiko, Hiroetsu

Wada, Jun

Wadati, Hiroki

Wade, Charles E.

Wagemaker, Tais Aleriana Lucon

Wagler, Jörg

Wagner, Andreas

Wagner, Anika

Wagner, Gabriele
Wagner, Pawel

Wahab, M. Farooq

Wähälä, Kristiina

Wahl, Patrick

Wakioka, Masayuki

Waksmundzka-Hajnos, Monika

Waldmeier, Felix

Walker, Heather

Walkinshaw, Malcolm

Wallace, David

Waller, Deborah

Wall-Medrano, Abraham

Wallner, Björn

Walsby, Charles

Walton, James

Walton, John

Wan, Chunpeng

Wan, Edwin

Wan, Jianbo

Wan, Shibiao

Wan, Xuehua

Wanders, Desiree

Wandzik, Ilona

Wang, Bibo

Wang, Bo

Wang, Bo-Cheng

Wang, Chao-Min

Wang, Chin-Kun

Wang, Chiu-Yen

Wang, Cunde

Wang, Fazuo

Wang, Feng-Sheng

Wang, Guozheng

Wang, Hsiuying

Wang, Hui-Min David

Wang, Jeffrey

Wang, Jian-Wen

Wang, Jianxu

Wang, Jimin

Wang, Juexin

Wang, Jun

Wang, Junjie

Wang, Kai

Wang, Lai-Xi

Wang, Lei

Wang, Li-Xin

Wang, Lizhu

Wang, Mengyu

Wang, Michael Cai

Wang, Mingming

Wang, Pengcheng

Wang, Zhengbao 
Wang, Qiuan

Wang, Qun

Wang, Richard R.-C.

Wang, Robert Y.-L.

Wang, Rongsheng

Wang, Ruibing

Wang, San-Lang

Wang, Shengnian

Wang, Sheng-Yang

Wang, Shih-Wei

Wang, Tong-Hong

Wang, Tsung-Jen

Wang, Tzu-Fan

Wang, Wentian

Wang, Xiaowan

Wang, Xinkun

Wang, Xinwen

Wang, Xiumei

Wang, Xiupeng

Wang, Xizu

Wang, Xuewen

Wang, Yi-Cheng

Wang, Yifei

Wang, Yu

Wang, Yun-Ming

Wang, Zeneng

Wang, Zhaohui

Wang, Zhe

Wang, Zhihan

Wang, Zhijun

Ward, Liam

Warnken, Zachary

Waser, Mario

Wasylka, Justyna Płotka

Watanabe, Hiroshi C.

Watanabe, Miki

Watanabe, Ryuichi

Watanabe, Shinichi

Wathes, Claire

Watkins, A. Neal

Watkins, Gordon Leonard

Waxman, Michael

Wdowin, Magdalena

Webb, Kimberly M.

Weber, Cameron

Weber, John

Webster, Ruth L.

Wege, Stefanie

Wei, Changyong

Wei, Cunxu

Wei, Hua

Wei, Jia
Wei, Kongchang

Wei, Qiang

Wei, Zhao-Jun

Weichert, Jamey

Weiss, Johanna

Welch, Alan

Welgamage Don, Aakash

Weller, Michael G.

Welling, Mick

Wen, Rong

Weng, Ching-Feng

Weng, Lihui

Weng, Meng-Shih

Weng, Xiaoyu

Wenland, Juergen

Wertz, Philip W.

Wesolowski, Marek

West, James D.

Westcott, Stephen A.

Westerlind, Ulrika

Westmark, Cara J.

Westwell, Andrew

Westwell, Andrew D.

Whelan, Rebecca

White, Jason C

White, Peter

White, Robert L.

Whitty, Adrian

Whorley, Sarah B.

Wianowska, Dorota

Wiczk, Wiesław

Widenmeyer, Marc

Widhalm, Michael

Wie, Myung-Bok

Wiedemann, Dennis

Wiedman, Gregory

Wientjes, Emilie

Wiesław, Wiczkowski

Wiesner, Silke

Wiesner-Reinhold, Melanie

Wiggins, Greg

Wijesinghe, Sudesh L.

Wijethunga, Tharanga

Wilding, Matthew

Wilds, Christopher J.

Wilkinson, J. M.

Willerth, Stephanie M

Williams, Elizabeth A

Williams, Gareth

Williams, Ian H.

Williams, Noelle S.

Williams, Paul J 
Willner, Itamar

Wills, Martin

Wilson, Anne M.

Wilson, David

Wilson, Heather L.

Wilson, Justin J.

Wilson, Thomas

Wilusz, Jeff

Winckler, Thomas

Winkler, Johannes

Wińska, Katarzyna

Witcher, Anthony

Witczak, Zbigniew

Witorsch, Raphael Jay

Wittstock, Ute

Włodarczyk, Maciej

Woelwer-Rieck, Ursula

Wójciak-Kosior, Magdalena

Wojciechowski, Łukasz

Wojcieszak, Robert

Wójcik, Grzegorz

Wójkowska, Dagmara Weronika

Wojnárovits, Laszlo

Wojtunik-Kulesza, Karolina A.

Wojtyczka, Robert

Wolf, Elmar

Wolfe, Lisa M.

Wolfram, Evelyn

Wolny, Juliusz

Wolny, Lidia

Wong, Chung F.

Wong, Clarence T. T.

Wong, Dominic W. S.

Wong, Man-kin

Wong, Shun

Wong, Vincent Kam Wai

Wong, Wai Shiu

Wood, Delilah

Wood, Troy D.

Woolbright, Benjamin L.

Woolridge, Elisa

Wöstemeyer, Johannes

Woster, Patrick

Woźniak, Łukasz

Wozniak-Knopp, Gordana

Wrigstedt, Pauli

Wu, Chi-Rei

$\mathrm{Wu}$, Chung-Yi

$\mathrm{Wu}$, Dayong

Wu, Hung-Chin

Wu, Jer-Horng

$\mathrm{Wu}$, Jiamin
Wu, Jian-Lin

$\mathrm{Wu}$, Jianming

$\mathrm{Wu}$, Jing-Yun

$\mathrm{Wu}$, Jin-Yi

$\mathrm{Wu}$, Li-Chen

Wu, Ming-Jiuan

$\mathrm{Wu}, \mathrm{Nan}$

$\mathrm{Wu}, \mathrm{Pao}-\mathrm{Chu}$

Wu, Pei-Fung

Wu, Ph.D., Wei

$\mathrm{Wu}$, Sheng-Chi

Wu, Shu-Ju

$\mathrm{Wu}$, Shu-Pao

$\mathrm{Wu}, \mathrm{Si}$

$\mathrm{Wu}$, Songlin

$\mathrm{Wu}$, Terence

Wu, Tian-Shung

$\mathrm{Wu}$, Ting-Feng

$\mathrm{Wu}$, Tongzhi

$\mathrm{Wu}$, Tzong-Yuan

Wu, Wen-Luan

$\mathrm{Wu}$, Xiangfa

$\mathrm{Wu}$, Xin

$\mathrm{Wu}$, Xin-Ping

$\mathrm{Wu}$, Xuejian

$\mathrm{Wu}$, Yunlong

$\mathrm{Wu}, \mathrm{Yu}$-Tse

$\mathrm{Wu}$, Zhanhong

Wu, Zhiqiang

Wuethrich, Alain

Wujec, Monica

Wulff, Jeremy E.

Wuttke, Stefan

Xavier, Cristina Pinto Ribeiro

Xavier, Nuno Manuel

Xhaard, Henri

Xi, Weixian

Xia, Junfei

Xia, Junfeng

Xia, Tian

Xiang, Chunhui

Xiang, Dongxi

Xiao, Dequan

Xiao, Kaijun

Xiao, Li

Xiao, Zhengtao

Xiaoli, Alus

Xie, Hong

Xie, Ran

Xie, Wankun

Xie, Wenyan

Xie, Yongshu 
Xie, Zhigang

Xie, Zhong-Ru

Xin, Dongyue

Xin, Hai

Xing, Chengguo

$\mathrm{Xu}$, Bin

$\mathrm{Xu}$, Chunfu

$X u$, Dazhong

$\mathrm{Xu}$, Jimin

$\mathrm{Xu}, \mathrm{Nan}$

$\mathrm{Xu}$, Peng

$\mathrm{Xu}$, Qinzi

Xu, Suowen

$\mathrm{Xu}$, Yan

Xuan, Junyu

Xuan, Tran Dang

Yabuki, Soichi

Yadav, Dhananjay

Yamada, Hiroyuki

Yamada, Koji

Yamada, Shuhei

Yamagishi, Takehiro

Yamaguchi, Kanji

Yamaguchi, Makoto

Yamaguchi, Masahiko

Yamaki, Kouya

Yamamoto, Yasunori

Yamashita, Atsushi

Yamashita, Teruhito

Yamato, Takehiko

Yamauchi, Satoshi

Yan, Bowen

Yan, Dayun

Yan, Dongqing

Yan, Jin

Yan, Jinhui

Yan, Shengmin

Yan, Xuehai

Yanai, Hikaru

Yanase, Emiko

Yancheva, Denitsa Y.

Yanda, Murali

Yandeau-Nelson, Marna D.

Yang, Chao-Hsun

Yang, Chia-Ning

Yang, Chun-Long

Yang, Ding-Yah

Yang, Feng-Ling

Yang, Hongshun

Yang, Inho

Yang, Kun

Yang, Lijiang
Yang, Seo Young

Yang, Seung Hwan

Yang, Shao-Yu

Yang, Sze-Ming Mildred

Yang, Tao

Yang, Teng-Chun

Yang, Wei-hsiung

Yang, Xinmai

Yang, Xiuwei

Yang, Yongkang

Yang, Zhibo

Yannakopoulou, Konstantina

Yao, Jiayi

Yao, Weirong

Yao, Yang

Yao, Zhili

Yaoita, Yasunori

Yaremenko, Ivan A.

Yasir, Iqbal

Yasuda, Shin

Yasuda, Takeshi

Yasui, Kyuichi

Yates, Edwin

Yates, Edwin Alexander

Yates, Matthew Z.

Yazdan Panah, Nima

Ye, Enyi

Ye, Huaiyu

Ye, Zhou

Yeh, Jan-ying

Yeh, Jwu-Lai

Yeh, Shu-Lan

Yen, Chia-Hung

Yen, Feng-Lin

Yen, Timothy

Yeo, Syn

Yerabolu, Jayasudhan Reddy

Yi, Myunggi

Yi, Tae Hoo

Yi, Tao

Yi, Young-Su

Yiannakopoulou, Eugenia

Yin, Sheng

Ylijoki, Kai E. O.

Yokohira, Masanao

Yokomatsu, Tsutomu

Yokoshima, Satoshi

Yokota, Kazushige

Yokota, Shin-ichi

Yokota, Shinso

Yokoya, Masashi

Yokoyama, Shinji 
Yonemochi, Etsuo

Yoneshiro, Takeshi

Yoo, Dongwon

Yoo, Ki-Oug

Yoon, Do-Young

Yoon, Hyeonseok

Yoon, In-Soo

Yoon, Jinhwan

Yoon, Kee-Dong

Yoon, Moon-Young

Yoshida, Hiderou

Yoshida, Kazunari

Yoshida, Kumi

Yoshida, Nidia Cristiane

Yoshikawa, Yutaka

Yoshimura, Morio

Yoshimura, Yuichi

Yoshinari, Nobuto

Yoshitaka, Yamaguchi

Yoshiya, Taku

Yoza, Brandon A.

$\mathrm{Yu}$, Cheng-Ju

$\mathrm{Yu}$, Chenglong

$\mathrm{Yu}$, Guoqin

Yu, Hongwei

Yu, Hua

$\mathrm{Yu}$, Jae Woong

$\mathrm{Yu}$, Jiayi

$\mathrm{Yu}$, Linlong

$\mathrm{Yu}$, Meifang

$\mathrm{Yu}$, Meihua

$\mathrm{Yu}$, Minzhong

$\mathrm{Yu}$, Rongmin

$\mathrm{Yu}$, Wenquan

Yu, Yan Ping

Yuan, Min-Hao

Yuan, Shinsheng

Yuan, Yaxia

Yuan, Zhihong

Yuan, Zhiqin

Yuan-Bin, Cheng

Yue, Patrick

Yue, Yanfeng

Yukl, Erik T.

Yun, Hyungdon

Yusa, Shin-ichi

Zabielska-Koczywąs, Katarzyna

Zaccheroni, Nelsi

Zacconi, Flavia C.

Zacharias, Martin

Zacharis, Constantinos K.

Zacharof, Myrto
Zaferanloo, Bita

Zagotto, Giuseppe

Zaharia, Valentin

Zahid, Maliha

Zaid, Hilal

Zaidi, Saheem

Zaitone, Sawsan A.

Zajdel, Paweł

Zakrevsky, Paul

Zakrzewski, Jerzy

Zalc, Bernard

Zaleśny, Robert

Zalubovskis, Raivis

Zamaratskaia, Galia

Zambrowicz, Aleksandra

Zamora Marin, Fernando

Zamudio-Rivera, Luis S.

Zamyatnin, Jr., Andrey A.

Zandarashvili, Levani

Zandi, Roya

Zapico, Sara C.

Zappia, Giovanni

Zappia, Stefania

Zaprutko, Lucjusz

Zaręba, Jan K.

Zarejousheghani, Mashaalah

Zarra, Ignacio

Zarrelli, Armando

Zarzycki, Pawel K.

Zastre, Jason

Zdarta, Jakub

Zeiner, Michaela

Zeitler, Axel

Zeković, Zoran

Zeleny, Reinhard

Zelkó, Romána

Zeng, Guangming

Zeng, Haishan

Zeng, Huaqiang

Zeng, Shuwen

Żesławska, Ewa

Zgórka, Grażyna

Zha, Shangwen

Zhang, Bin

Zhang, Bo

Zhang, Diming

Zhang, Duo

Zhang, Fan

Zhang, Fuming

Zhang, Gang

Zhang, Hengyou

Zhang, Hongqiao 
Zhang, $\mathrm{Hu}$

Zhang, Hua

Zhang, Huawei

Zhang, Jianying

Zhang, Jie

Zhang, Jingjing

Zhang, Jinnan

Zhang, Junbo

Zhang, Junran

Zhang, Junzeng

Zhang, Kai

Zhang, Ke

Zhang, Ming

Zhang, Pangzhen

Zhang, Peng

Zhang, Qianqian

Zhang, Qichun

Zhang, Qing-Wen

Zhang, Qiong

Zhang, Ruiyong

Zhang, Run

Zhang, Shaozhong

Zhang, Tao

Zhang, Wei

Zhang, Wenheng

Zhang, Wujie

Zhang, Xiaoming

Zhang, Xuanjun

Zhang, Xueqiang Alex

Zhang, Xueqing

Zhang, Yaolin

Zhang, Yingyue

Zhang, Yixiang

Zhang, Yixuan

Zhang, Yu

Zhang, Yunkai

Zhang, Zhaowei

Zhang, Zhenbin

Zhang, Zhong-Yin

Zhao, Jin-Hao

Zhao, Liang

Zhao, Ruiming

Zhao, Shan

Zhao, Xiaoai

Zhao, Xinxin

Zhao, Yuxia

Zhao, Zongmin

Zhbannikov, Ilya

Zhdanov, Vladimir P.

Zheng, Liqiu

Zheng, Shilong

Zheng, Xiaolong
Zheng, Xinting

Zheng, Zhaoliang

Zhong, Haizhen

Zhong, Tuhua

Zhong, Wei-Zhu

Zhou, Bangjun

Zhou, Carol L. Ecale

Zhou, Dezhong

Zhou, Guo-Ping

Zhou, Haibing

Zhou, Jia

Zhou, Lili

Zhou, Shengbin

Zhou, Shuanhu

Zhou, Xiaoshun

Zhou, Zhe

Zhu, Changfu

Zhu, Feng

Zhu, Liangdong

Zhu, Shan

Zhu, Wei

Zhu, Xiaoshan

Zhu, Ying

Zhu, Zhenwei

Zhukov, Igor

Zhukova, Natalia

Žiarovská, Jana

Ziegler, Joerg

Ziegler, Thomas

Ziegler-Borowska, Marta

Zielinska, Beata

Zielińska, Sylwia

Zielińska-Jurek, Anna

Zielińska-Pisklak, Monika A.

Zierkiewicz, Wiktor

Zimmer, Elizabeth

Zimoch-Korzycka, Anna

Zinchenko, Anatoly

Zingales, Sarah

Zingue, Stéphane

Zinna, Francesco

Ziora, Zyta

Zitko, Jan

Zivcak, Marek

Zloh, Mire

Zlotek, Urszula

Zollo, Massimo

Żołnowska, Beata

Zolotoukhina, Tatiana

Zoltan-Istvan, Szabo

Zorc, Branca

Zorc, Branka 
Zotin, Alexey A.

Zou, Gang

Zou, Ping

Zou, Quan

Zovko Končić, Marijana

Zsuzsanna, Bősze

Zubkova, Olga

Zucca, Paolo

Zuluaga, Paola

Zuo, Ran
Zuo, Yuegang

Zupko, Istvan

Zuriaga, Elena

Zuvela, Petar

Zych, Maria

Zygmunt, Magorzata

Zygmunt, Małgorzata

Żymańczyk-Duda, Ewa

Żyżelewicz, Dorota

(c) 2019 by the author. Licensee MDPI, Basel, Switzerland. This article is an open access article distributed under the terms and conditions of the Creative Commons Attribution (CC-BY) license (http://creativecommons.org/licenses/by/4.0/). 